






\section{DE LA FORIILTION}

ET UE LA

\section{FÉCONDATION DES EUFS}

CIIEY LES

VERS NÉMLTODES 



\section{DE LA FORIIATION}

\section{V.T IN $1 . \mathrm{A}$ \\ FÉGONDATION DES CEUFS \\ (1I)Z LES \\ VERS NEIIATODES \\ PAR}

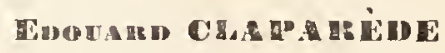

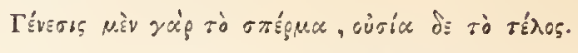

Aristoteles, De parlibus animulium. A. 7.

GENEVE

IMPRIMERIE DE JULES-G' ${ }^{\text {me }}$ FICK

1859 



\section{DE LA FÉCONDATION DES ALLS}

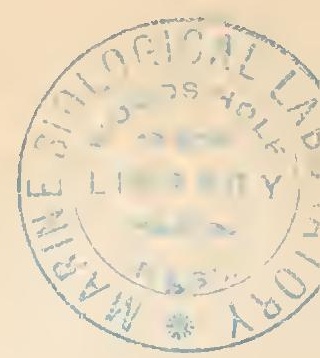

\section{CHEZ LES VERS NÉMATODES.}

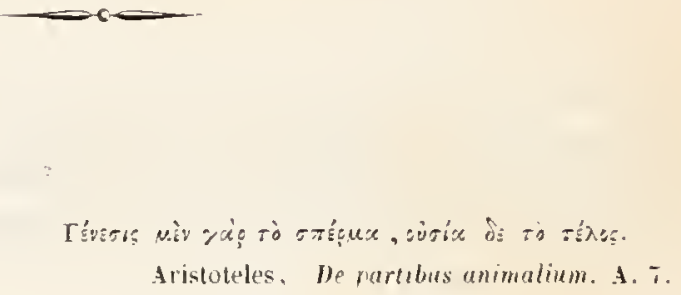

REMARQUES PRELIMINAIRES.

Ce mémoire a élé rédigé dhuant l'hiver de 1836-18:57 et remis le 50 Avril 1835 an secrétarliat de l'Université de Berlin, pour concomir an prix proposé par la Finulté de médecine de Berlin pour le meilleur mémoire sur lat question traitée daus les pages qui suivent. Il a été couromné le ã Aoǹt de li mème amnéc et, bien que onze mois se suient. éconlés entre ce moment el celui on nous lo lirrous à l'impression, il n'a subi depuis tor's ancun changement C'est ce qui expliquera pourquoi quelques travaux qui ont paru daus l'intervalle ne sont point mentionnés dans ees lignes. Nous sommes toutefois heureux de pouroir citer encore ici les observaltions intéressantes yu’un sivant lien conmu, M. Otto Funke, a publiées en 1857 sur la question des 
prétendus connles épithéliaux et sur la genèse des corpuscules séminaux de 'Ascaris Myslax'. Ces observations concordent de tous points avec les notres, et il est heureux de voir deux observatemrs parfaitement impartiaux et inconnus l'un à l'autre, venir simultanément se prononcer d'une manière concordante dans une question si vivement lébattue.

Nous avons du reste publié dlans l'intervalle un résumé de ce mémoire dans le Journal dirigé par MM. de Siebold et Kölliker ${ }^{2}$.

Genève. Juillet 1858.

I Lelırbuch der Physiologie für akademische Vorlesungen und zum Selbststudiun von Dr Otto Funke. Bd. II. Leipzig 1857, p. 1299 et suir.

${ }^{2}$ Ueber Eibildung und Befrnchtung bei den Nematoden. Vorläufige Minheilung von E. Clit-

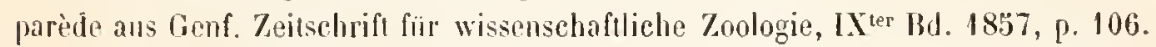




\section{PARTIE IISTORIQUE.}

Lorsquion se propose d'eximiner avec sang-froid ef eritique une question qui a suscité des discussions vives el acerbes, il est urggent. de s'armer de toutes pièces, d’ébulier avec soin l'histoire génétique de la question elle-mime. On apprend par li de quelle manirire certains ćléments plus ou moins hétérogènes sont venus s'introdurire là oì ils n’avaicnt que finre, et quelle est l'importance qu'il fint leur attacher. Le fait même qu'une question est discutée avec aigrenr el passion, suthit à ourrir la porte ì une multitude d'erreurs qui, pour l'observateur impartial, anmaient éte des plus faciles à éviter. C'est, en particulier, lì ce qui est arrivé à propos de la question de la formation des aufs et de la fécondation chez les vers nématodes (Nematoda). Le sujel u'est point en lui-mème fort cmbronillé, bien qu'il oflre des difficulcés assez nombreuses; mais les savants matmralistes qui hui ont consacré, durant ces derniires amnées, tout leur zèle et souvent anssi toute leur fougue, en ont fait un vai labyrinthe dans lequel il est aussi difficile de retrouver le fil conducteur que dans cehui de Crète. Un savant réputé, Allen Thompson, s'est proposé dernièrement pour but de replacer la question sur son vai terrain el sons mu jour plus propice. Son opuscule, conç arec calme el dignité, constamment assisté d'une observation consciencieuse, est, à notre avis, ce qui a paru de mienx sur le sujet. Néanmoins le dernier mol n’a point encore été dit par lì dans ce long débat, d'antant plus que le protesseur de Glasgow ne connaissait pas encore, au moment où il réligea son mémoire, les observalions récentes de Schucider, qui, pour peu qu'elles dussent se confirmer, pourraient ébranler de nouveau l’élifice si péniblement reconstruit. Voilà pourquoi nous jensons pouvoir venir, is notre tour, mrendre la parole, esperant contribuer, dams la proportion de nos forces, ì faire avancer le prohìne d'un pas et le dépouiller de quelques-unes de ses dilficultés. 
Les anciens ohservatemrs, y compris Rulotphi el Cloquel, ne se sont oecupés que de la distribution macroscopique des organes génératems, "l cest seulement à partir d'Henle' al d'Eschuch" que nous trouvons une élude réelle du rontenu dr ces organes. Ces salvants recommurenl que les wuls soml, chez beancoup d'ascarides, rémis on paquets formant des esprees de grappes on d'éloiles. Mais ce n'est, dans le fail,

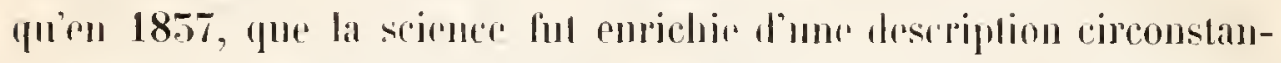

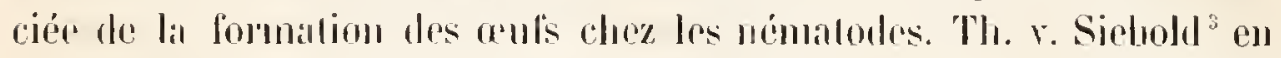
éail l'anlem. Il crul recommaille que, chez celte classe te vers, les

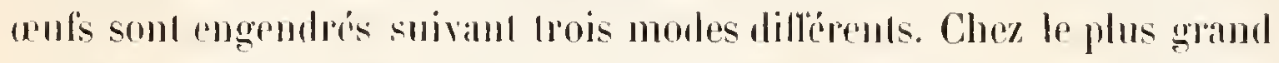
nombre, il troure la partic postérienre (ćest-i-dire, lesmince en cap(am) de l'ovare remplic partun contenu vésiculeux el incolore, anquel

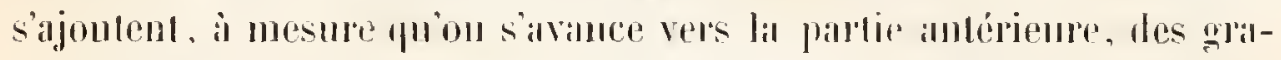
mules exeessivement pelits. Ces glinules qui ne sonl anlle chose qur

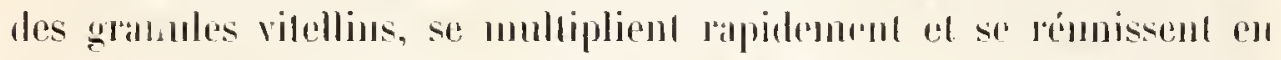
masses aromdies, qui sont les premiers rudiments dos ants. Ces masses se pressent les mes contre les antres, siphlatissent el forment alors comme des piles d'ócus. (C'est a ce moment, suivant de Sichohl, quiapparaissent, pour la premiere lois, la vésicule de Purkinje el la lache germinative.) Les ants insi formés amivent enfin dims l'ulérus. Le second type, représenté par l'Ascurislumbricudes, est cantaclérisé par le lait que les vents rudimentaires ne lindent pas il prendre ume forme irrégulierenent pyramidale. Les sommels des pyramides salrangent

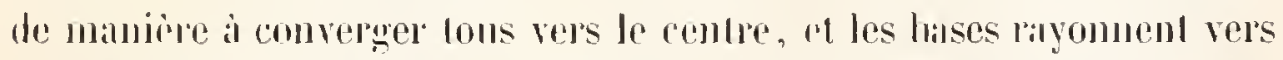
la périphrérie. C’est a re moment-la pue se nomlroul la vésicule el la lache germinative. Enliu, dans le troisième lỵe, représenté par l'Ascaris ancta, les gramules vilellins so réunissent daus l'ovairo en masses anrondies qui se peconrenul d'une pellicule mince; phos has, dans If lube ovarien, ces masses s'associent par groupes de cing ou luit on davaulage, en s'mussanl entre olles an moyen de fils délieals. Nous

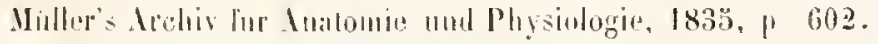

"Foriep's neu" Jolizen 1848. N. 318. 1. I47.

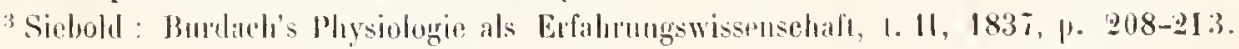


anrons l'occasion de voir phus loin que ces dommés nécessitent certaines rectilications, en particulicr pour ce qui concerne la réunion des auts en groupes'. Siebold déerit dans le même onräage le fluénomène de la segmentilion des auls chez les livers nématodes.

Les résultats de ces observalions gaguèrent hientôt en extension, matce a la dissertalion de Bagge, impriméce en $\mathbf{1 8 4 1}$ à Erlangen. Son autem a m le mórile de laire commaille les zoospermes des nématodes, si différents, d'après ses jecherches, de ceux des amtres amimanx. Il recomut, en effel, que ces zoospermes ne sont point des éléments mobiles flus ou moins filiformes, mais des corpuscules globulenx privés de lont noourenent. Cela n'enpêcha point que, dès l'annce suivante, Nayer ${ }^{3}$ prétendit avoir tronvé des zoospermes filiformes chez une oxyure (Oxyuris vermicularis). Séduit par ce précédent, liöliker aima mienx prèter l'oreille à une théorie altrayante, que de s'en tenir à ce qu'il royail de ses yeux, et bien qüil n’eù jamais vu lui-même de filaments mobiles daus les urganes générateurs des nématodes, il ne vouInt trouver dams les corpuscules séminamx de Bagge que des faisceaux de spermalozoïdes filiformes. Celte manière de voir a été dès lors comballue a juste litre pall Reichent ${ }^{5}$, Sichold ${ }^{6}$, Nelson ${ }^{7}$.

On pent dite que, depuis Bagge, l'état de nos connaissances ne s'est

1 Siphold a lui-mème rectific plus lard ce demier point, en re sens qu’il a reconnu que les coufs soit de l'Asearis auclit, soil de l'A. lumbricoides, soit de différents aulres nématodes A. Mystas, A usculata, Cucullanus elegans, Strongylus inllexus/ne sont poinl isolés les uns des antres dans l'o aire, mais sunt gronpés auton d'un rachis central anquel ils adhèrent intimemenl. Tergleichende Anatomir, 18is, 1. $13 \%$.

2 bisceraltio inanguralis de andutione Strongrli auricularis et Ascaridis acuminatas. Er-

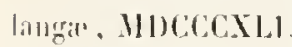

3 Tene Cntersuchungen aus den liebiete der Anatonie und Physiologie, 1842, p. 9.

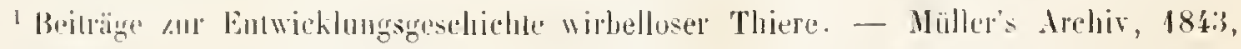
1.. $68-1$ il

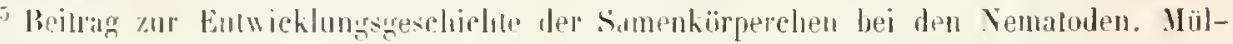
trer's Arehis, 1847, j. 90.

6 Vergleichende Anatomie, 1848, 13d. 1, 1. 133, Anmerkung 8.

"The reproduction of the Ascaris Yystax. - Philosophical Transactiuns of the Royal Suviety, 18.32. Pirt. 11. 
pas amélioré d'une manière bien rẻjonissante. Des observalions nombreuses ont été faites de côtés divers sur la formation soil des auls, soit descorpuscules séminaux, chez les némaloïles, mais ces olservations sont, en général, assez contradictoires. Kölliker étudia la genèse des reuts chez diverses espèces, et arriva à un résultat assez différent de celui que Sichold arail lail connaitre. Il considère la tache germinative comme l'élément primaire, le punctum saliens de la lormalion de l'oul'. Les taches germinatives se trouvent isolées, sous la forme de gramules élémentaires de grosseurs diverses, dans la partie li plus reculée de l'ovaire. Chacun le ces gramules s'entoure plus lard d'une pellicule, el devient par hi tune vésicule germinalive, ornée de sa lache caractíristique. Celle vésicule elle-mème s'enveloppe de granules vitellins: et de celte manière se forme l'aul' complet. Reichert ne se joignil point complétement ì l'opinion de Kößliker, car, an lieu de ne tronver, comme celni-ci, dans la partic avengle de l'ovaire que des gramules ćlémentaires, il y rencontra des cellules toules lormées, les cellules-mères des oul's.

La formation des zoospermes a ćté décrite par Sicbolıl chez l'Ascaris pancipara . La partic aveugle du testicule est, d'après sa description, remplie par des nucléus pourvus chacun d’un mocéole. A mesme que ces mucléus descentenl rers le bas du iesticule, ils s'entourent l'umc substance limement granuleuse, fui clle-mème finil par s'envelopper d’une membrane lénur, si bien que le testicule ressemble alors ì un ovaine rempli d'uul's, lont chacm anurait sa vésicule el sa lache germinative. Les mucléns des cellules ainsi formées moditient leur forme, s'allongent el premuent des routours phus décidés. Reichent" dans un travail cironstancié sur la formation des corps séminau cliez les nématoüdes, conteste ces données de Siebold. La partic areugle du testicule renferme, selon lui, non point les mucléus de cellules appelées ì se former plus loin, mais les cellules véribules ol complètes, pui sont, ì ses yeux, les cellules-mires d'autres cellules. Ces dernicres sont les germes des zoospermes (Keime der Spermalozoen).

1 Vergleichende Anatomie, p. $1: 33$

${ }^{2}$ K. B. Reichert, Beitray zur Enwicklungsgeschichte der Samenkiorperchen bei den Nematoden. M. Arch. 18:7, p. 88-14\%. Tab. II 
Un mémoire de Nelson sur la reproduction de l'Ascaride du chat (A. Myslax), mémoire qui parul en 1852', ouvil une nouvelle ère dans la question qui nous uccupe, et domna lieu à une longue suite de débats yui n’est pas lerminéc à l'henre qu’il est. Ia manière dont Nelson représente la formation les zoospermes rappelle plassablement te mode suivant lequel Kö̈liker veul avoir olsservé la première formallion des aufs. Lextrémité du lesticule" ast, suivant hui, homogène ì l'exlérienr,

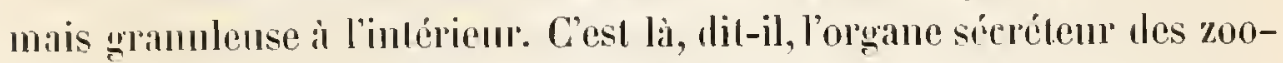
spermes; en effet, les grimules se délachent de la paroi, s’enllent el se trausforment en cellules mucléées qui llollent lans un liquide transparent. Entre ces cellules se trouvent des granules fins, dont le nombre va lapidement en augmentant. Les gramules se groupent antonr iles cellules el forment ainsi antour de chacume nne enveloppe épaisse qui, d'abord grande et irréguliere, prend pen à pen des dimensions moindres el s'armondit. Les cellules spermatiques ne se développent pas davanlage dans l'intéricur des organes màles. Mais Nelson les retrouve dans l’ipparreil génital des lemelles, oi alles subissent des modifications sensibles. D'abord leur enveloppe granuleuse disparait. Elles se présentent pall suite sous la lorme de cellules claires douées d'un, parfois de deux nucléus discoïdaux. Puis, le nucléns croil en dimensions el se limite par une membrane du côté où il tonche à la paroi de la cellule, de manière à ôtre distinctement séparé de celle-ci. La membrane ne larde pas i sélendre antonr du nucléus, en lormant une espèce de capnchon conique ou mieux te cloche. Le mucléus ainsi translormé est le zoosperme mùr el propre à la fécondalion. Il se libère de la cellule enveloppante et altend le moment de jourer son rôle.

Pour bien saisir la manière dnont Nelson croil que la lécondalion s'opiore chez l'Ascaride du chal, il nous lant encore passer en revue le mode génétique que ce savant altrilme amx oufs. Nelson fail naître les orules dans l'oraire, précisément comme les cellules spermalipues dans Ie testicule. En effet, dams la partic en coecum ale l'organe il trouve dos

\footnotetext{
1 Loc. cil.

2 Loc. cil., p. :36.j.
} 
"granules qui se tuméfient el deviennent de véritables cellules : les vésicules germinatives des aufs à venir. A mesure que ces vésicules descendent vers les régions inférienres de l'ovaire, elles s'entonrent de gramules sécrélés par les parois du lube ovarique. Ces granules, qui ne sonl lien autre que des granules vilellins, s'agghomèrent antour des visicules ef domnent ainsi maissance ì des aufs dépourvus de membranes vitellines. Lesœuls, toujours parfaitement mus, arrivent dans la partie du tube gémérateur que Nelson désigne sous le nom d'oviducte, et li ils rencontrent les zoospermes. La fécondation sopère. Les zoospermes, bien que privés de tout monvement, pénètrent dans les ouls, dont la surfice inerme ne lemr oppose pas de résistance, et, à partir te ce moment, les oufs sont aptes à su développer. Les cellules spermatiques qui out pénétré dans l'intéricur des aufs perdent leur forme caractíristique. Elles se mélamorphosent en corps irréguliers transparents el tris-réfringents, tamdis que les anfs enx-mômes s'entomrent d'me donble membranc. Le professeur Allen 'Thompson avait permis à l'antemr de le citer comme témoin d'une grande partie des observations dont nous venons de dommer une breve esquisse.

Le travail de Nolson méritait bien certainement d'iltirer l'attention du public savant. On y reconmaissait la main el l'esprit d'un observateur consciencieux. Puis, il soulevait de nouveau la querello famense des spermatistes el dles ovistes. Déjì en 1842, Barry avait prélendu avoir observé la pénétration des zoospermes dams les aufs de lapins, mais celto assertion ne paraissait pals aroir trouvé grande créance daus le monde physiologique. Cependant les olservalions de Nelson sur la

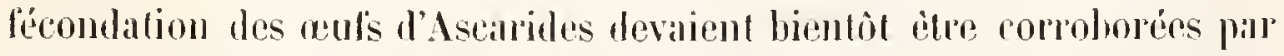
d'autres analognes, linies par Newport chez les Batraciens of par Keber ${ }^{3}$

${ }^{1}$ Researches in Embryology. Thirl series. A contribulion to the Playsiology of Cells. Ihil

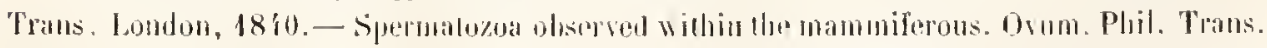
1843.

2 On lle impregnation of the Oum in the Amplibia second series) and on lhe direct Agency of llue Spermalozoa. Phil. Th. 1833. Part. II.

3 Leber den Eintrilt des Samtenzellen in das Ei, ein Beitrag zur Physiologrie der Zengung. könirsberg, $18: 33$. 
chez les amodontes et les lapins. Si celles de ce dernier soulevineul déji

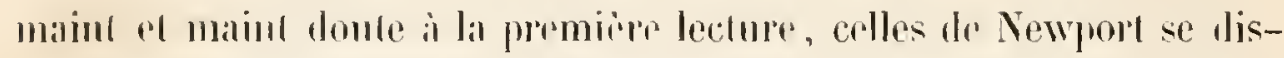

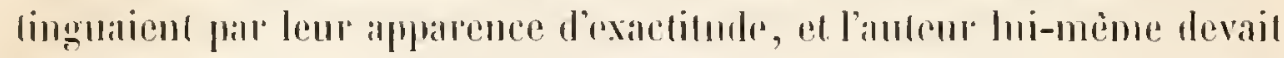

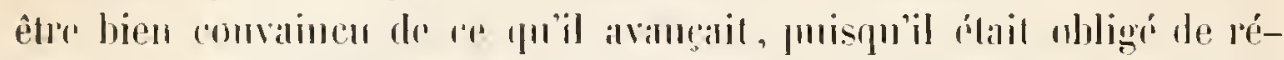

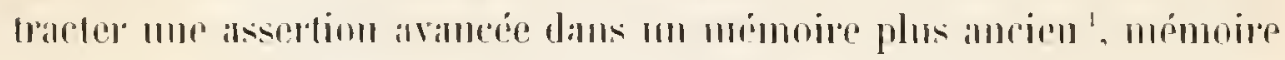

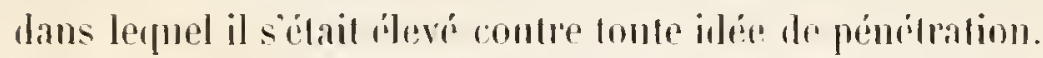

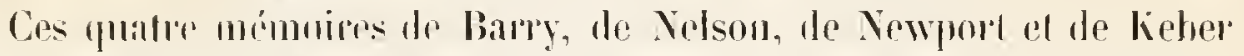

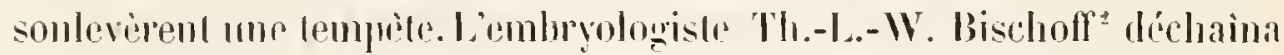
sur enx loutes ses fomlers el Jem dénia de lons juinls les résullats aux-

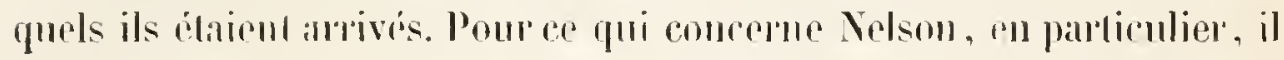

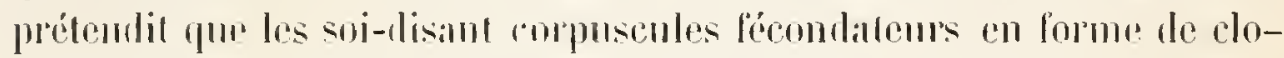

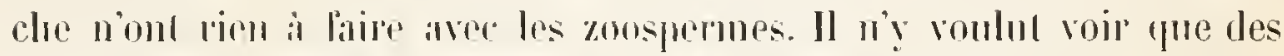
formations ipithéliales, des rommles ápithólianx (Epillıelialliegelchen).

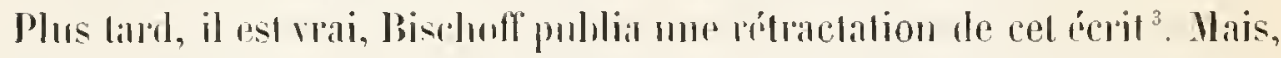
dans cet ofuscule on il reconnait aroir en tort daus ses atlapues contre Barry el Yewport, il mainlient complétement son premies dire vis-ivis de Nelson. Leurliant assure égalemenl s'itre convancu que les corpuscules en queslion sont allorents ì la partie interne du tube oraripue.

Ce fut alors que (ieorge Meissmer appanul sur la scine. Dams um premier mémoire concernant un rer appartenant il lorde des Gorliacés Mermis albicans), il exposil, sur la formalion des aufs el des zonspermes, des vues qui s'ámitient assez de colles armises jusqualors chez les mématodes proprement dis. Dans un second mémoire", il pril

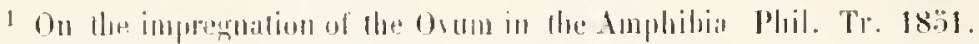

2 Widerlegung des von 1$)^{r}$ Kicber hei den Najaden um 1$)^{r}$ Nolson hei den Ascariden behaup-

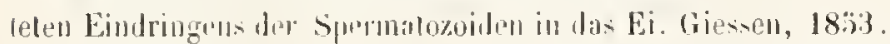

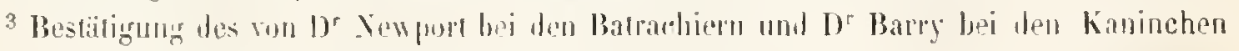

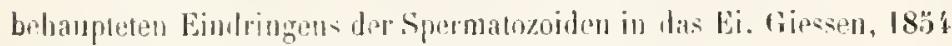

4 Leuckart \%nzat\% z.n lischofl's Widerlegung.

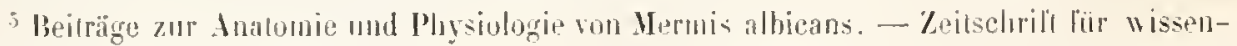
schaftliche Zoolorio Bd. I. Hece 18\%3.

"Beobachungen ïber das Eindringen ler Sanmelemente in den Doller No I. Zeitschrilt 1. wiss. Zoulogie, Bd. 11. Sept. 18:3'. 
pour ohjec de ses recherehes un rai némaloule l'Ascaris Mystax du chat, el il se joignail a Velson porm voir dams les comules épithélianx de Bischolf les véribbles zoospermes. En oulbe. Meissner émellat mo lhéo-

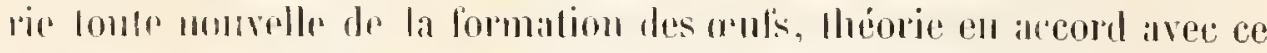

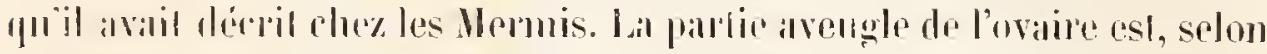
Ini, remplie de cellules, qui soml les cellules-mines des and's. Cenx-ci se forment eomme andand de direrticules de ces cellules, direrticules yni

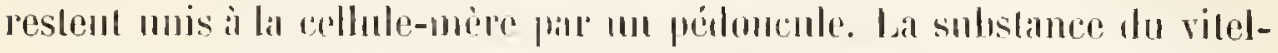

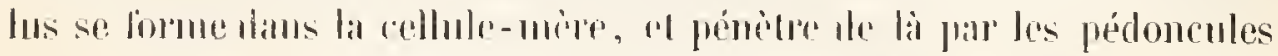

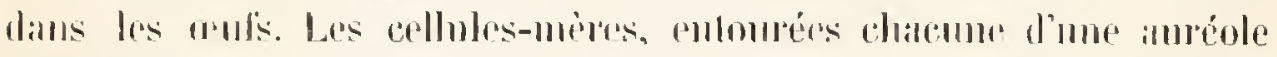

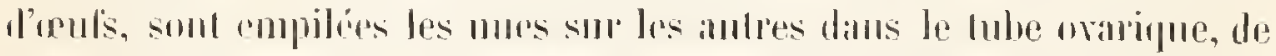

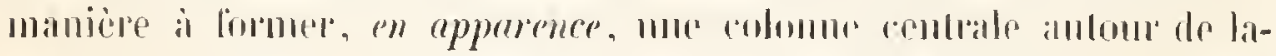

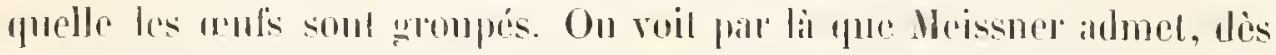

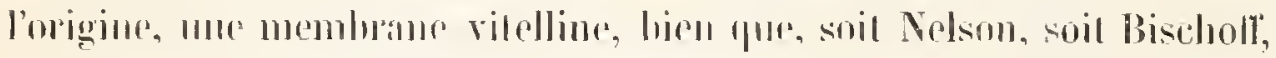

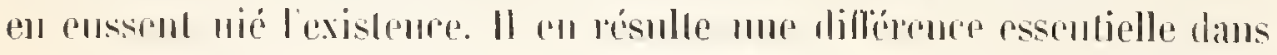
la suile de lévolulion de lexnll, snivant Meissner. En elled, il vient m inourent mi les aruls se délachend de la cellule-mire. La place par la-

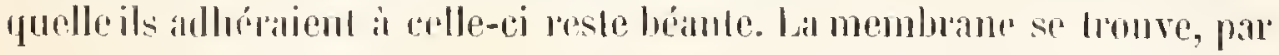

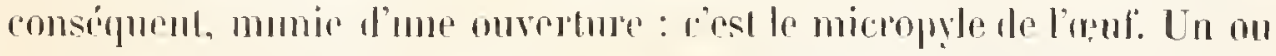

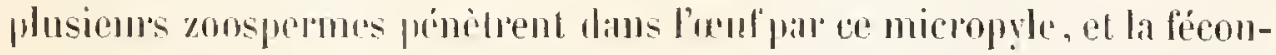
dalion se troure ainsi oprón. Le sorl ullériem res zoospermes almis

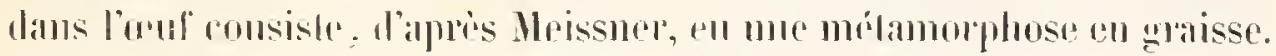

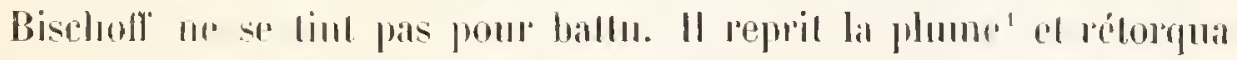

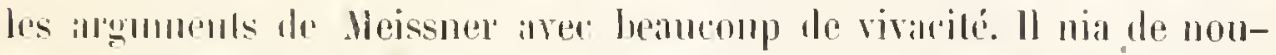
veall que les corpusenles en forme de chochr fissent des zoospermes,

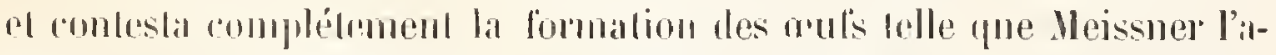

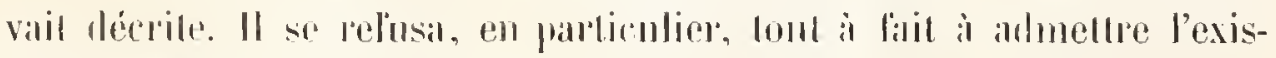
tence des rellules-mires el de leurs diverlicules, d dichan que les

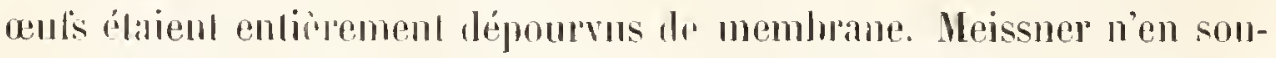
lint pas moins som dire, ol, dans un mómoire bresétendu sur les Gor-

\footnotetext{
1 Bischull: Lober Ei- Inul Samenbildung und Befruchung bei Asearis Myslad. Zeilsehrift I. wiss. Zoulurie. Februar 18:5:)
} 


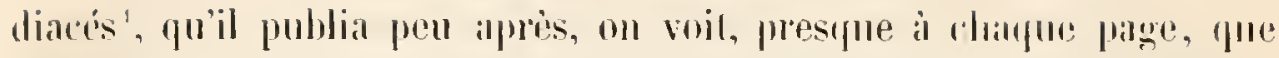

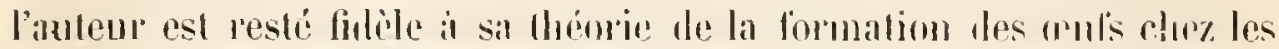
Mermis el chez l'Asearide du chat.

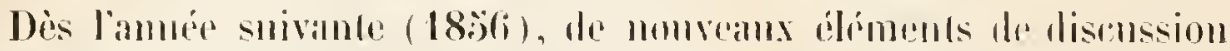

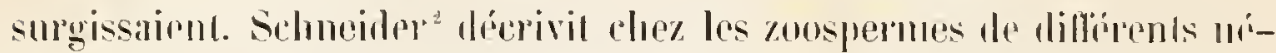

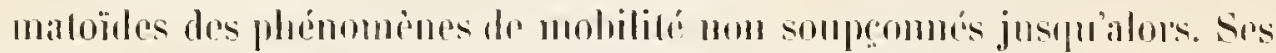

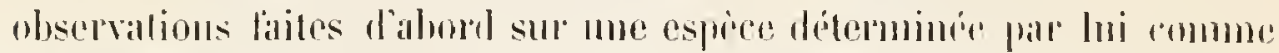

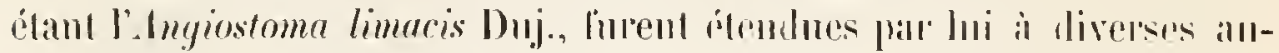

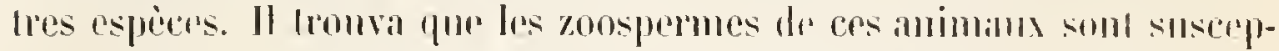

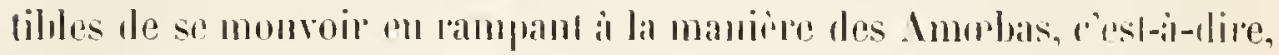

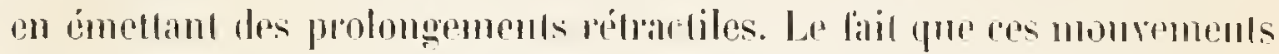

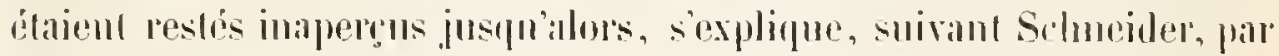

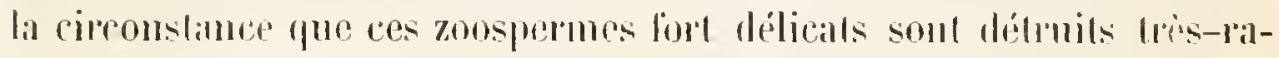

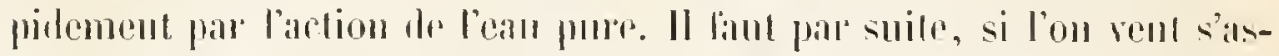

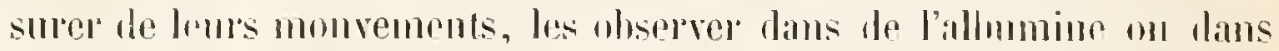
ume dissohution de sel millin.

Schneider évitail aree pmolence dre se prononcer sur le déball entre Nelson, Ireissner ef Biseholt. On en comprend aisément lit canse. En eflet, il n'álait proint encore démontró que les sinģuliess phénomènes observés pan lui lussent rais pour lous les mémalodes, ol il n’avait

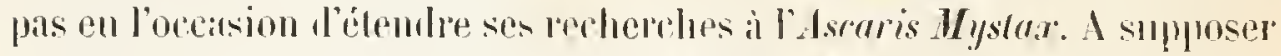

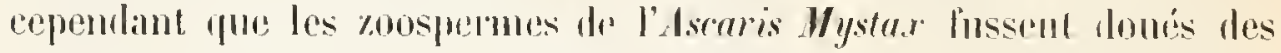

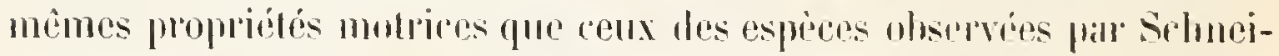
der, il devenail assez probshlo qure Bischofi avait raison dins lo débal,

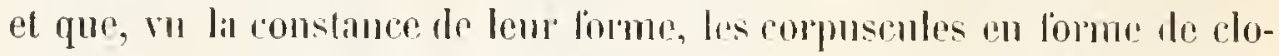

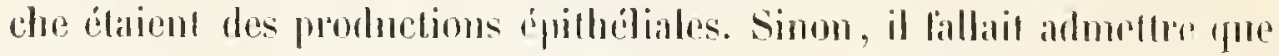
la forme de cloche n'est pue la lorms: quillectenl los zoospermes à l'étal de repos, el que ees corpuscules soul susceplibles, à un moment rlomé, de prendro me lome irréguliore of de se monvoir is la maniere des Amolias.

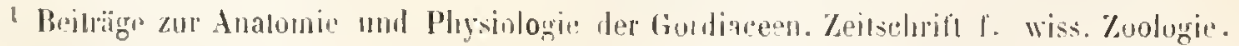
Nai $18: 3 \%$.

2 Leber Rewerung an den Samenkiorputen der Nematoden. Munalsberich der Berliner Atadenie, 1836, p. 192.
} 
Dernièrement, enfin, "nn mémoire d'Allen Thompson' est renu re-

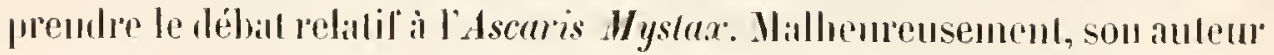

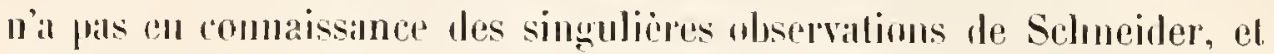
I'a pas fu, farr ronséquent, chercher à fos concilier alser ses propres idées. Thompson, comme nous l'aroms deejai dit, Iraile lil question avec

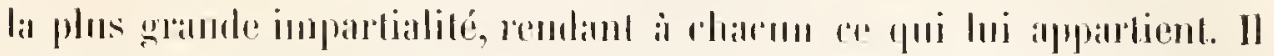
rejelte les comules épilhéliamx de Bischolf, el domme rason à Nelson el à Jeissner, qui en font des zoospermes. Eur revinche, il combat la théorie de la lormalion des auls souleme par Neissner, el prélend,

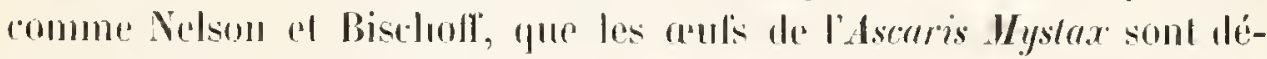

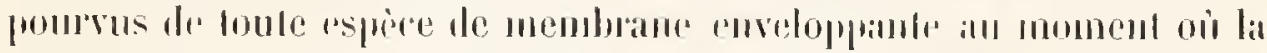

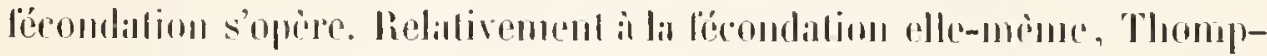

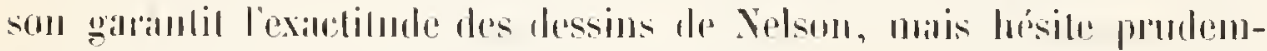

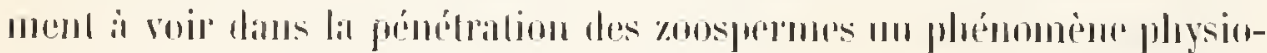

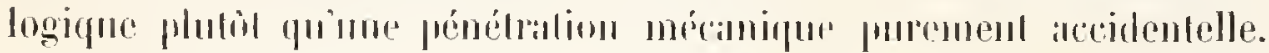

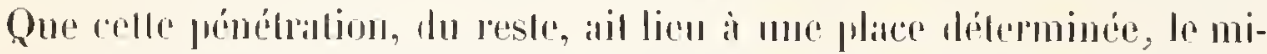

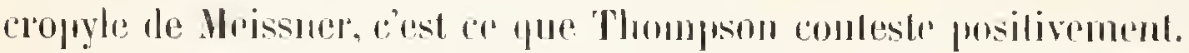

Tel est lital de la puestion all nomment oi noms furemons la plume. Bien des primls, on le voil, sonl encore enlonis dans le dounte des conleshations. Volson, Veissner, Biseholf, Schmedider forment chatcun un

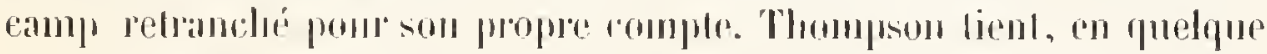

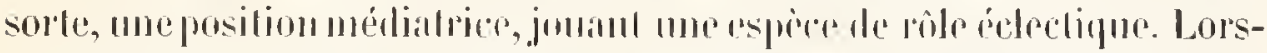

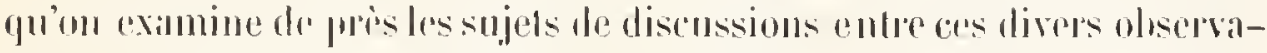

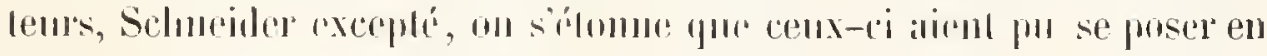
innlagonistes illssi decidés les uns des antres. Sumvent ils onl tous plus

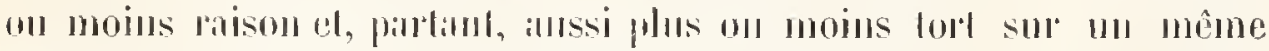
poinl. C'est li le cats, fril exemple, pour ce qui concene l'existence on labsence d'une mombrane vitelline ì un stade douné du développement de l'eul'. Thompson, exemph de tonte infóe préconcue, a su se montrer plein d'éputé et de justice vis-a-ris do chacun des combattants. Espérons que nons santrons liare do même.

I Ueber die Samenhämperchen, die Eier und die Befruchlung der Asea ris Mystan. Zeil-

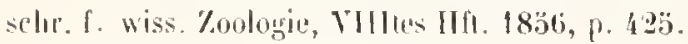




\section{I1. HISTOI.OGIE DES ORGANES GENERATEURS.}

Il esl urgent, en abordant le débil de notre sujed, de commencer par

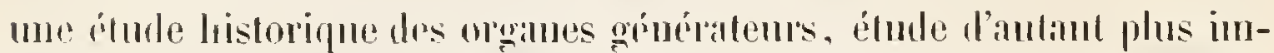
portantr qu'elle pent nous lournir le moyen d'édatireir vertains points du dibint sonleve mutr Nelson, Meissner el Bischoll. Une grande partie de ce débat, en effet, repose sur la puestion de savoir s’il existe, oui on non, chez les nématodes un épithélium cylindrique, ronslitué comme les corpuscules en lormo de ploche de l'Asciride du chal.

La plupirt des anteurs so sout contentés lle domner une destription macruscopique des moines générateuts des mímatodes. Nous nous borurrons a indiquer, il cel igaril. que nous conserverous les termes em-

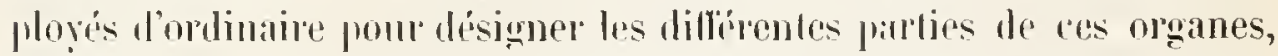
à savoir, chez les femelles: ovaire (baslogine of vitellogène), vésicule séminale ( qui u'se pas toujours présente), trompe de Fallope on ovilucte, utérus of viginin; chez les miles: lesticnle, camal délïrent, vésicule steminale el conduit ajaculaleur. Nons rejetons fo nom d'albuminogène (Eiweisschanch), employé par Meissner, faree qu'il nons pilrail fort douteux que la substance sécréce par la partie de la trompe le lallope yu’il désigno sous ce nom, soil bien réetlement de l'albmmine.

Külliker, le premier qui se soit ucenpé do l'histologie du bube ovarique chez les némalodes, a prétendu ' quo l'extrémila avengle de l'ovaire (en particulier chez l'Ascaris dentula) ast composér d'ume simple rangée de cellules placées hout i bout, dont les parois se résorbent aux points d'allérence réciproque, dr sorte quil se forme un lubr coutinu. Lo-

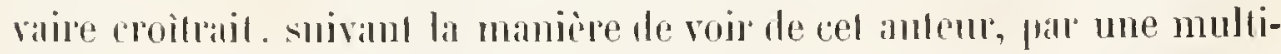
plication endogène de la cellule terminale. Nous comsidérous cette opinion le Kö̈lliker comme manpuant de tont fomdement. II est probable que ce sivim s'est laissi sédmire pill des imiges lrompenses dues à des

$$
1 \text { bue. cit., p. 70-71. }
$$


actions diosmotiques. Il sulfit, en effet, de laisser ighil quelque temps de l'ean pure sur l'ovaime l'un ver nématöde prom voir se protnire des figures rappelant lout ì lail celles domnces pall liölliker. C'est ce qu'avail déji reconmu Ricicherl. Ce lernier pense que les parois du lube sexuel so composent dhez les nématodes l'mo membrane simple, mince, lanspatrente el en apparence diponrone de loulo struchere, membrane qui se continne, me lois arrive a l'orilice généralemp, dans la combe de substance conjonclive des parois rlu corps. II en conchul que celte membrane doit aroir la mème valeur histologique que la substance conjonctive elle-mème. Quant à nous, nous trouvons, en général, le tube ovilripure des nématodes composé d'unc membrane sans structure dẻmontrable, qui sépaissil souvent assez consilérablement au fond de l'extrémilé arengle. Nous avous représente celte place épaissie clicz le Cucullams elegaus (PI. IV, Fig. 1, a), chez nne ascarile de lintestin grile llu Tritom taniatus (PI. VIII, Fig. 11, a), ele. Parlois, Yéparississement prend les proportions très-consilipables, comme nous

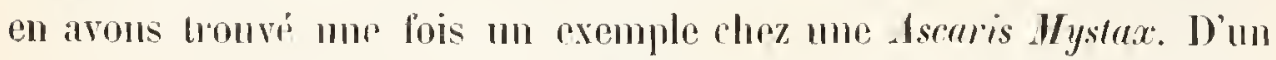
antre cóté, il n'est pas tres-rare de rencontrer des individus chez lesquels la membrane n'est pas plus épaisse au lond du coenun que partout ailleurs.

Cependant les parois do tube ovirique ne sont point formées miquement par ceth: membrane sans structure apparente. La surface in-

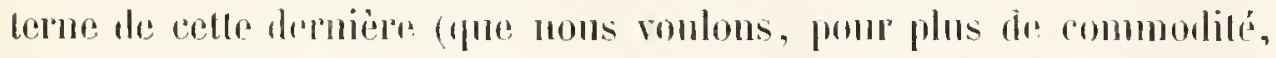
désigner sons lo nom de tmrique propre) se revil d'un Imnique imlime, lormér par une conchr épilhóliale. Cé épithélium est toujours facile à reconnatio daus tos partics du tube sexuel qui sont te phus voisines de l'ouverture sexnclle, a savoil, dans le vagin el daus l'ulérus; mais il laut dijà plus d'altention pontr le retronver dans l'oviducte, la poche séminale et l'waile. Nous ne sommes mème jamais parvemu à suive les cellules épithéliales jusqu’au sommet proprement dit de ce dernier, c'est-i-mlire, jusqu'i son extrémité avengle. Les meléns, plus faciles i 
recommintre que les collules elles-mêmes, deviemuent d'ordinaire plus rares et moins dislincts, a mesure fu'on se rapproche du sommet de lovaire. puis entin ils disparaissent romplétenrent. L'ápilhólimm n’est, du

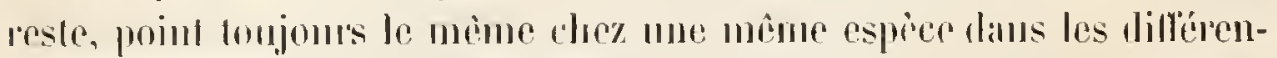
les pallies du lubr sexuel. Ainsi, par exemple, diez le Curullams elegans l'épilhélimm de la parlie de l'ovidncte qui est siluée mole la poche séminale al lutérus, est composé de pelites cellules oruces de gros

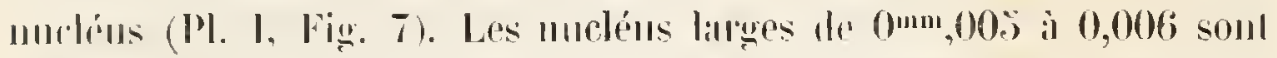
mime si gros relativement anx cellules, quils remplissent presque complétement colles-ci. L'épillélimm de la vésicule siminale el de la

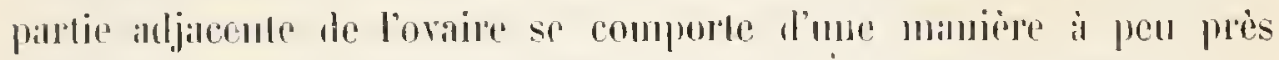
semblable. Par contre, lasped de la lonigne intime est lonl antre lans l’ulérus. Iorspue relui-ci est contractí, on ne reconmair te läpillélium quo les mucléus dont la couleur est un pen plus claire que celle du reste de Porganc (PI. I, Fig. 6) el des plis longiludinan. Mais, dans les moments d'expansion de l'orone (Pl. I, Fig. 1), un peul distingutu les trails fins el déliés qui délimilent les cellules épilhéliales. Ces collules so dessinent, en parliculier, trés-ulictinctement lorsquou les colore par de la teinlure d'iode très-iblendur. On reconnail alors que les

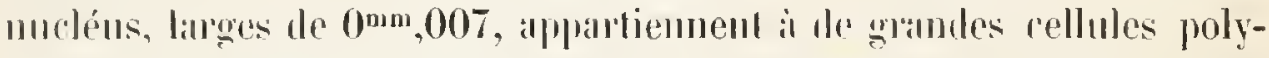
groniales, longues en moyenne de $0^{\mathrm{mm}, 02}$ ì 0,05. Lépillaélium du lube sexul est, commu l'ou voit, un éfrithélimm pavimenteux; les cellules

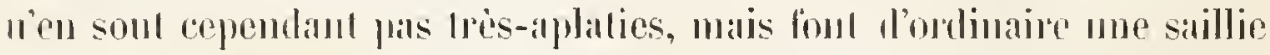
assez semsible dims l'intérieur du lube. Elles soml mumies d'un un-

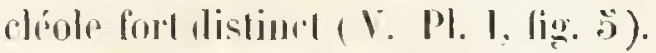

Il s’agil mainlenanl de savoir sil existe due les mémalonles des productions épilhéliales coniques, Ielles que celles qur Bischofl prélend

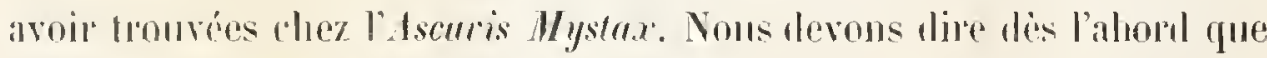

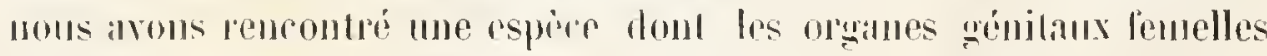

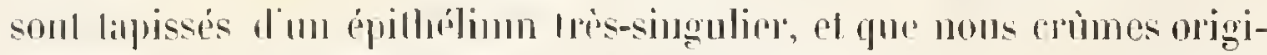
nairemenl voir li une prenve en favenu de Bischoll daus la puepolle de celni-ci aver Nelsou a Meissner. Celle esprice esl l'Ascuris smilla de l’inlestin du porc. Chez celte ascaride, soit les mérus, soil lit partie des ovi- 
ductes qui est adjacente à ceux-ci, sont lapissés par de très-grandes cellules f́pithéliales, dout la longuem varie entre $0^{\mathrm{mm}}, 10$ el 0,18. Chaqur cellule émel de som milien me sorte de stolon conique qui fail saillie dans le lube sexucl (V. PI. I, lig. I et 2). Les stolons ont une hauleur a $0^{m m}, 018$ a 0,027 . Lem largem valric à peu pres entre les mômes limiles. Les uncléus des cellules ne sont pas liaciles à apercevoil

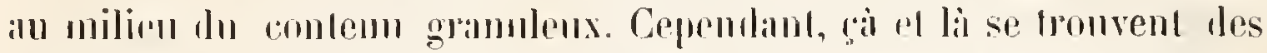

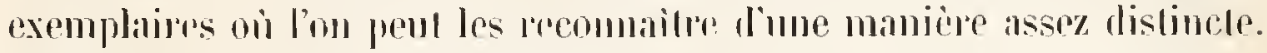
Ces sholoms roniqurs lappellent jusqu’à un cerlain point par loum forme les commles épillóliams dr Bischofl, avec celle différence qu’ils sont

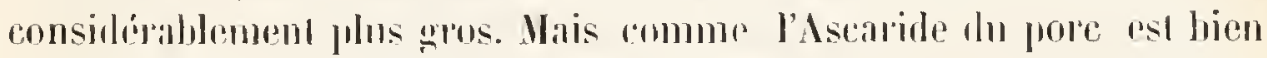
phrs gramde qur colle alu chal, yni fit lobjel des recherehes de Bischoff, ce fail ne serail bas unr oljection lien forte. II es aussi juste de remarquel que l'alhésion des comules épilhéliamx le Bischoff à la membrane de lovidncte doil ètre des plus délicales el des plus fragiles, si bien que, suivant Bischofi, on troure on général, pall suite de la préparation mème, la plupart des combles arrachés à leur base. Il n’en esl poinl ansi des slolons coniques de l'tscaris suilla; lonl an contraire: lem mpmbrane est la continualion immédiate de la membrane de la cellule. On a beau diehiber les parois du hule sexnel en lons sens arec

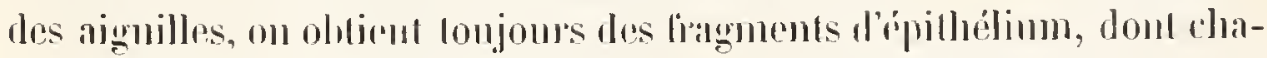
que cellule est sulmontée de sou stolou, mais jamais dr stolon isolé,

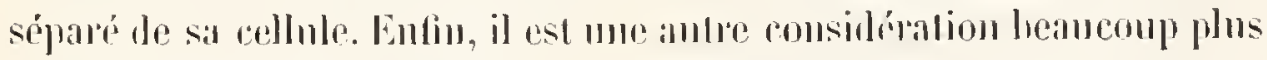

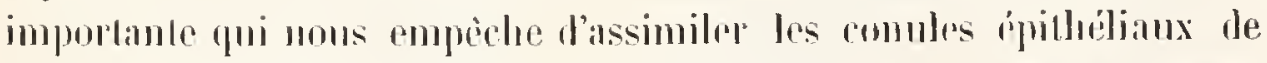
Bischoll a ces produclions évilemment épilhéliales de l'Ascaris suilla : C'est que les soj-disinl romules ípilhcilianx se tronvent en onlre des stolons chez les indivilus fennelles pui ont dé fécondés, ef nons anons loccasion de dimuntrep sultisimment plus loin que res commles nomt. rien ì laire arre l'épithélimm, mais pu’ils sont les vébiables zoospermes, comme Nelson, Meissner a Thompson l'onl driji recounu. Ces zoospermes (V. Pl. V, fig. 10) sont de bien phus pelite taille yue les stolons.

Meissner" mentionne déjì chez l'Ascar is megalocephala du cheval 
des cellules épithéliales munies de villosités, ou prolongementsen forme le langue. Il est fort rraisemblable yue ce sont li des productions tontes semblables ì celles que nous venons de lécrire. Meissner, il est vai, n’en signale l'existence que dans l'albuminogène (Eiweisschlauch), tandis que nous tronvons ces cellules appendiculée, chez l'Ascaris suille, non-senlement dans le soi-disant alluminogine, mais encore dans l'ovilucte proprement dit et dans l'ulérus.

Il est probable rne les singulières cellules de l'ntérus ef de la partie inférieure ale l'oviducte chez l'Ascaris suilla sont doućes llune fonction spécialc. Ce sont elles, sans ancun donte, yui sécrètent la substance servant ì formel l'enveloppe externe des aufs. C'est, en effet, seulement ì partir de la place où l'oviducte est tapissé de ces simgulières cellules épillıéliales que les œufs commencent ì s'entourer de cette enveloppe. On ne peut pas dire non plus que Bischolf ait réuni ensemble deux objets qui n’avient rien de commum, qu’il ait confontu sous un même litre les pronluclions épithéliales et les zoospermes. En effet, e'est en vain que nous avons cherché chez l'Ascaris lu chat (A. Mystax) un épillélium semblable ì celui de l'A. suilla. L’utérus est tapissé de grandes cellules avec mucléns et nucléoles, précisément comme le décrit Nelson"; souvent les cellules ne sont pas dès l'abord trìs-faciles à distinguer, mais on reconnait toujour's facilement les plis de l'utérus semés de nucléns nucléolés (P). I, fig. 5). Que ces plis soient, ainsi que Bischofl l'aflirme², hérissés de pscullospermatozoüdes, c’est ce que nous n'avons jamais pu voir. L'ovidnete est pourv'u d'un épilhélium analogue ì celui de l'ulérus; les mucléus y sont ovales et lonģs de $0 \mathrm{~mm}, 0 \mathrm{~L} 5$ ì 0,022 .

Meissner parle clıez le Mermis nigrescens ${ }^{3}$ de cellules aplaties et nucléées qu'il a tromvées sur la face interne de la tunique propre de l'utérus. Ce sont évilemment les cellules épithéliales qui viennent de nous occuper. Il ajoute que ces cellules ne forment point une couche

1 Loc. rit., p. 371 .

2 Bischofl's Widerlegung, p. 29.

${ }^{3}$ Beiträge zur Anatomie und Physiologie der Gordiaceen. Loc. cit, p. 36. 
continue, mais qu'elles sont disposćes en bandes longitudinales. C'est lì une disposition que nous n'avons rencontrée chez aucun némaltode. Nous avons au contraire toujours vu l'épithélium former une couche uniforme sur tonte la surlace de l'organe. Chez les Gordins, Meissner a du reste trouvé un épithélium contimu. Lieberkïhn a décrit avec beaucoup de soin la manière dont l'épithélimm est distribué chez un nématode, qu'il a tromvé dans le proventricule le la Fulica atra et de l'Anas Boschas². II n’a pas non plus rencontré d’épillélium dans la partie supéricure du tube générateur. Schneider a décrit un épithélium hexagonal dans le tube générateur de l'Angiostoma limacis ${ }^{3}$.

L'utérus et le vagion s'entomrent en général chez les nématodes d’ume tunique externe, de nature musculaire. Les fibres musculaires ne sont pas toujours très-faciles à constater, mais l'adjonction d'une petite quantité d'iode suffit sourent à les faire recommaître, parce que les fibres se colorent d'un brum plus intense que la substance interjacente. Dans les grosses espices, comme l'Ascaris lu porc on mème celle du chat, la nature musculcuse de la couche externe est facile à constater. Mais cest clez l'oxyure de l'ho mme (Oxyuris vermicularis) que nous arons pu étudier le plus commodément les museles de l'utérus et du ragin. Les fibres musculaires y sont disposées transversalement autour du lube génératenr ì une cerlaine distance les unes des autres, et forment des anastomoses entre elles (PI. I, fig. 8). Ces fibres sont si épaisses qu'elles font saillie sur le contour de l'organe. On les roil se raccourcir et sallonger pendant les mourements péristaltiques du tube généraleur. Il est bon de dire que les mourements péristaltiqnes de l’ulérus et du vagin s'exículent avee une viracilé loule particulière chez l'Oxyuris vermicularis.

Le vagin, l'utérus el l'oviducte paraissent jouir plus on moins chez tous les némalodes de propriétés contractiles. Toutefois on ne rénssit point chrz lous ì reconnaitre l'existence de fibres musculaires dams les

1 Beitr. z. A. u. 1. der Gordiacen. Loc. cil. 11. 11 .

2 Beitrïgezur Anatomie ler Nematoden. - Müller's Arehiv 185\%, 1. 314.

${ }^{3}$ Lue. eil., p. 192. 
parois de ces organes. Sonvent la conche contractile ne laisse reconnaitre en fait de structure qu'me consistance gramuleuse, comme c'est, par exemple, le cas chez le Cucullaus elegans. Il est cependant souvent impossible de ne pas reconnaitre que les granules de celte couche sont disposés d’une manière plus ou moins évidente en raies transversales. Pent-ètre que certains róactits chimiques réussiraient à faire voir des filnres répondant à ees stries de gerains. Meissner ' a déja fait mention chez le Mermis nigrescens at chez les Gordius d'une conche contractile de l'uténs, dams laquelle il n'a pas pu recomaitre de structure particulière.

Enfin, nous arous à mentionner des bourrelets longitudinaux el gratunleux qui ont été vus par tous les observateurs au tube ovarique de I'Ascaris Mystax et que nous retrouvons tout semblables chez l'Ascaris suilla (V. Pl. II, fig. 26). Ces bourrelets larges et lígèrement obliques à l'axe de l'ovaire font saillie a la surface interne du tube sexnel, et sont semés de granules qui ressemblent beaucomp aux gramules vitellins. La partie de l'ovaire qui est mmie de ces hourrelets élant précisément celle on le vilellus se forme, le vitellogine, Nelson a suppose ${ }^{2}$ que les bourrelets longitudinaux sont l'organe sćcréteur du vitellus. Meissner, ru sa théorie de la formation des oufs, a rejeté cette idée, et Thompson ${ }^{3}$ ne s’est exprimé quavec beaucoup de réserve à cet égard. Il nous semble donteux que ces bourrelets on còtes longitudinales jouent le rôle qui leur est attribué par Nelson, parce que nous n'avons jamais pu nous assurer que des granules s'en détaclassent pour aller se rémir aux rufs, of il ne nous semble pas impossible qu'il faille y voir un appareil confractile analogue aux stries gramuleuses transversales du Cucullanus elegans.

La constitution histologique du tube générateur màle est parfaitement analoğne ì celle du tube générateur femelle. Ici anssi nous tronvons une membrane propre sins struchure appréciable, dont la surface interne se recouve d’une couche épithéliale, et la surface externe, du

1 Reiträge zur Physiologic und Anatomie der Gordiaceen. Loc. eit., p. 36 el 103.

Loc. cil., P. ว̈

${ }^{3}$ Loe cil., 1. $43 \%$. 
moins dans les régions inférieures de l'apparcil, dune couche musculaire. La parlie areugle du lesticule est souvent épaissie, mais d'ordinaire moins considérablement que la partie correspondante del'ovaire. Il ne nous a jamais été possible de reconnaitre l'épitlélium dans la partie du testicule qui avoisine l'extrémité aveugle. Nous avons représenté l'épiohélium du conduit éjaculatem d'une ascaride de la lotte (Lola vulgaris), ascarile qui est sans doute l'Ascaris moronata de Schrank (V. PI. I, fig. 9). Les nucléus des cellules sont excessivement gros, alteignant un diamitre de $0{ }^{\mathrm{mm}}, 008$ ì 0,006 , c'est-î-1lire plus de la moilié de celui de la cellule. Dans la vésicule séminale du mìle de beiucoup d'espèces, les cellules épithéliales atteignent au contraire de grandes dimensions relativement au nucléus el an nucléole.

La couche musculaire externe se comporte précisément comme chez les organes femelles. Chez l'Ascaris suilla le testicule présente des còtes longiludinales granuleuses analogues ì celles y̧uou trouve chez les femelles. La résicule séminale possède par contre à sa surface externe un róseau de fibres contournées en sens divers. Ces fibres ont une lar-

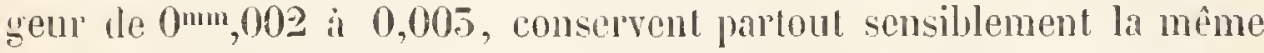
lirgeur et contiennent gà et là des granules. Enfin il est des espèces oì l'on ne peut recomnaitre ancune structure appréciable de la couche contractile, comme c'est le cas pour le Cucullams elegans.

Chezl'Ascaris mucronala de l'intestin de la lote, la conche musculaire revèt une apparence toute particulière. Le conduil ajaculateur est reconvert à sa surface externe de cellules musculaires liusiformes trèsallongées. Les nucléns de ces cellules sont ovales et fort gros, alteignant jusqu’ì $0^{m m}, 016$ en longueur. Ils renferment constamment un ou plusicur's nucléoles. Nous reparlerons plus loin de ces cellules musculaires des nématodes.

Les organes copulatemrs màles offrent quelques particularités intéressantes. Chez diverses especes on trouve ì la base des orgames comnus sons le nom de pénis ou de spicules ume aggonomération de cellules. Ces cellules, an nombre de trois ou quatre, atteignent parlois une dimension raiment élonnante. l.es phus grandes que nous ayons vues 
appartenaient it un ver trouvé daus l'intestin d'un pore el qui paraissail ètre un jeune individu de l'Ascaris sulla. Lal plus grande le ces

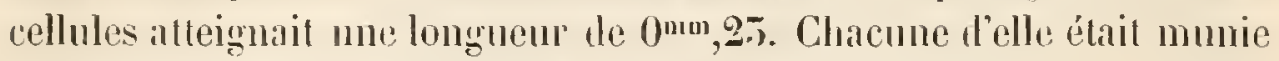
d'un nucléus clair (V. P. II, fig. 2, B), antour duquel étail concentrée une substance granuleuse. L'action de l'acide acétique faisait ressortir plus clairement encore les nucléns et montrait dans clıacun d'eux un uncléole, renfermant hui-même un petit corpuscule (PI. 11, fiğ. 5). Les nucléus avaient un diamétre d'environ $0^{\mathrm{mm}}, 01 \mathrm{~g}$. Le pénis el ces cellules sont enveloppés par un fourreau commm (Fig. 2, e) qui se continue tans le perinysium (Fig. 2, a) des muscles rétracteurs (Fig. 2, c). Chique spicule est muni de deux muscles rétracteurs ou tilıres rétractrices, légèrement bifurqués au point où elles s’insèrent ì l'oregane copulateur. Chaque fibre se ramilie phisieurs tois (Pl. II, lig. 1) avant d'aller se fixer anx parois du corps. Ces filores ont mo structure tres-complexe. Dejji it laide l'un l'aible grossissement on y distingue un cylindre médullaire plus obscur (Pl. II, fig. I, c) et me conclie corticale plus transparente (Fig. 1, c), comme cela a lieu chez beanconp d'animaux inférieurs (ćchinodernes, artlropodes, elc.). A un grrossissement plus considérable on conslate que la substance mélullaire es granuleuse (Fig. 2, e), mais que les granules sont disposés en raies de manière i constiucr des stries Irausversales. Des places ovales el claires (Fig 2 , e), longutes de $0^{\mathrm{mm}}, 01$, sont disséminées dans la moëlle, surtout dans la partie qui aroisine les grosses cellules. Ces places claires disparaissent complétement lorsquion s'approche des ramifications des filures. Sous l'action de l'acide acétique, une ou deux de ces taches prennent des contours décidés (Figy. 2, d) et se donnent i recomnaitre comme des nucléus munis de leur nucléole. Mais la plupart (Fig. 2, c) conservent leurs contours indécis. La substance corticale est strice an long (Fig. $\mathbf{2}$, l) et paraì être formée de librilles longitudinales. A sa place la plus large le cylindre médullaire atteint une largem de $0 \mathrm{~mm}, 06$, el la substance corticale une épaisseur de $0^{\mathrm{mm}}, 018$. La fibre musculaire ainsi formée est cnveloppée d’une tunique légèrement plissée à lapuelle on pent domner le nom soil de sarcolemme soit de perimysium, car on pent anssi 
bien considérer celte fibre comme un muscle à structure complexe que comme une fibre isoléc. Celte tunique se continue, comme nous l'arons dit, dams le fourreau des cellules et ilu spicule. Ce fourreau est muni cà el là l'un nucléus ovale (Fig. 2, f). Celte conformation des muscles rétracteurs des spicules est d'autant plus intéressante que celte ascaride ne possède pas d'autres museles de structure semblable. Les bandes musculaires longitudinales les parois du corps sont de larges faisceaux de fibres très-minces, dims lespuels il n'est pas possible de distingurer les couches que nous venons de décrire. En somme, les muscles des nématodes offrent une diversité de forme réellement incroyable, conme nous le montrerons plus loin.

Le spicule renfermé dans le fourreau se compose lui-mème de cing membranes en forme de tube iplati, emboitées les mes dans les autres. La premire (Fig. 2, gr) est mince et légèrement plissée comme le périmysium. La seconde (Fig. 2, h) est épaisse el lisse. C'est elle qui donne an spicule sa fermeté el quon considère en général comme formée de cette substance si vague à laguelle on donne le nom de chitime. Elle se dissont cependant i clatud dans la potasse canstique. La froisième (Fig. 2, i) est composíe de gros granules. La quatrieme (Fig. 2, k) est lisse et trìs-lịnement striéc en long. La cinquième (Fig. 2, l) forme l'axe du spicule, el il est dithicile de dire si c'est un tube membraneux rempli de liquide on un solide de révolution homogène. l'acide sulfurique colore en jaune intense les granules de la troisiome membrane. En mème temps se produit une coluration d'un violet superbe qui paraì avoir son siége dans la première et la quatrième membrane, mais qui se commminue rapidement an liquide ambiant, par suite de la dissolution de la substance colorée. Du reste, cette coloration n'est que passagère. An bout de dix minutes ou un quart d'henre il n'en restait plus trace.

On trouve à la base des spicules d'un grand nombre de nématodes des renflements assez consilérables, et il est probable que ces renflements seront toujours formés par rle grosses cellules comme celles que nous venons de técrire chez l'Ascaris suilla. Guido Wagener, auquel 
nous commmiquàmes notre dessin, nous dit avoir dẹji reconnu clıez diverses espéces que les rentlements en question renlerment des éléments celluleux, siurs en avoir éludié les délails d'une manière exacle.

Chez l'Ascaris mucromata da li lote les cellules de la base des spieules (Pl. VI, fig. 5, b) sont fort grandes. Elles sont relativement beaucoup plus étroites et plus longues que chez l'Ascaris suilla. Elles ont environ la moitié de la longueur des spicules. Ceux-ci mesurent 0mm, 56 . Chaque cellule est mmic d'un fort gros nucléus (Fig, 5, c) contenant parfois jusqu’à 150 nucléoles el au-dessus. Ceux-ci so dissolvent ou du moins deriennent invisibles dans l'acide acélique concentré. Le fourreau du spicule (Fig. J. c) est excessivement large, paree qu'un corps gramuleux (Hig. 5, i) se loge daus son intérieur à coté du spicule. A la surlince externe du fourreau se trouvent ippliquées quelques cellules musculaires lusiformes (Fig. $\overline{3}, \mathrm{k}$ ) ì long uncléus dont l'action est sans doute antagoniste de celle des muscles rétractenrs, et a pour eflel de laire saillir le spicule. Le spicule ne se compose chez cetle espèce que de trois membranes invaginces les unes dans les aulres. La membrane méliane est celle qui domne an spicule sa consistance. Elle est fincment dentelée à l'extrúmité qui lonche aux grosses cellules (Fig. $\bar{y}$, g). Soit la membrane interne ( Fig. 5, h), soit la membrane externe (Fig. 5, f). sont munies de plis transversaux qui sont plus marqués dans la première que dans la seconde.

Quant à la fonction des spicules, elle n’est pas toujours très-évidente. It ne semble an effet pas possible que les spicules servent dans tous les cas anssi directement fu’on le pense en général à l’intromission de la semence dans le vagiu, ear its ne sont certainement pas toujours canaliculés en gouje. Du reste, cette explication fait délaut dans les genres où il n’y a fưm seul spicule, el où ce dernier n'est ni camaliculé ì sa surlice, ni percé d'un canal central. Clıez les espèces munies de deux spicules inégaux, il n’est cerlainement pas possible que les teux pieces s'appliquent l'une contre l'antre, le maniòre ì laisser un canal entre elles. Chez les cucullans, Dujardin' admet mn spicule sim- 
ple accompagné en arrière par une pièce accessoire plus petite, servant de gaine partielle. Mais celle description n'est point très-exacte, du moins pour le Cucullamus elegans des poissons d'eau douce. Il y a de fail deux spicules semblables (V. Pl. II, fig. 6), dont l'm est considérablement plus petit que l'autre. Lorsque le plus grand est poussé en dehors, il ne glisse point sur le plus petit. C'est tantôt le spicule droit, tantôt le gauche qui est le plus grand, mais c'est plus sourent le ganche. En somme, il parait licite de supposer que les spicules jouent souvent simplement le ròle d'oryanes excitateurs, tout en facilitant accessoirement lintroduction de la semence.

Chez hearcoup de nématodes mâles, probablement mème chez le plus grand nombre, la partie du corps qui avoisine l'ouverture sexmelle est semée de pelites verrucosités. On connaît dẹjà de semblables particularilés clıez heaucoup d'espèces. Meissner s'en est occupé en détail chez les Gorliacés. Mais nous les retrouvons chez des espèces oǹ nons ne croyons pas qu'on les eût mentionnées jusqu’ici. Ainsi, par exemple, chez le Cucullams elegans, à la face inférienre dnquel nous trouvons de claque cité de la ligne médiane une rangée d’une douzaine de petites vertues ou papilles (IP. II, fig. 6, c). Ces verrucosités sont sans dloute les ouvertures de petites glandes. On rencontre du moins des espèces clicz lesquelles il n'est pas donteux qu'elles ne soient percées d'un canal central. C'est le cas, par exemple, chez l'Ascaris suilla, qui présente de chaque còté de si partic postéricure denx rangées de vertucosités, l'une plus longne, l'autre plus courte (V. Pl. II, fig. I, e, e’). Les plus grandes atteignent $11 n$ diamètre de $0^{\mathrm{mm}}, 04$; les plus petites seulement de 0,02 . Immédiatement à côté de l'ouverture anale, qui est la mème que l'ouverture sexuelle, se trouvent des verrucosités munies de deux ouvertures. Un certain nombre de stries transversales du tégument sont interrompues hrusfuement par la présence de ces organes (Fig. 1, e' et fig. 5). Ces petites glandes ont probablement pour but de sécréter une sulıstauce propre à faire adluérer plus facilement le màle ì la femelle pendant l'accouplement. 
Revarove. Nous voulons profiter de l'oceasion pour ajouter quelques mots sur certains points de Thistologie des nématorles qui ne rentrent pas précisément dans notre sujet, mais qui n'en sont prs moins d'un lout intérêt. Dans ses différents travaux sur les Gordiacés, Meissner a décrit chez ces animaux des musples longitulinanx, sans pouvoir reconnaitre l'existence de muscles 1ransversaux. En revanche, il a déconvert un système nerveux très-complexe, et il a figuré la manière dont les fil.res nerreuses périphériques vont se confondre avec les fibres musculaires qu elles coupent presque a angle droit. Sans vouloir contester positivement l'exactitude des observations de Meissner, nous ne pouvons noms empêcher de dire que nous avons conçu certains dontes il leur égard. Meissner lui-même dit que crrtains anteurs, comme Siebold, ont pris les nerfs pripiphériques pour des muscles. Or, il est maintenant aréré à nos yeux que, sinon chez les Gordincés, du moins chez les Nímatodes proprement dits, les organes en question sont des muscles et point des nerfs. Lieberkühn a déja déerit ' exactement la manière dont les muscles transversaux se comportẻnt relativement aux muscles longitudinaus chez un nématode, parasite de la Fulicu atru. Mais il est des espècrs qui conviennent encore mienx ì une étude semblable. Nons arons trouré, par exemple, en grande abondance dans le Tiriton temiatus à Berlin une ascaride non encore arrivée à maturité sexuelle, dont les muscles longitudinaux sont composés de cellules fusiformes allongées et très-distinctes (Pl. IIII, fig. 12). Ces cellules ont somvent une longueur do $0 \mathrm{~mm}, 39$. . Chacune d'elle est munic d'un gros nucléus ovale, atteignant une longueur de $0^{\mathrm{mm}}, 0 \mathrm{z}$, et renfermant un ou deux gros nucléoles. Les cellules musculaires sont unies les unes avec les autres par des commissures étroites et déliées (Fig. 12) qui s'élargissent un peu an point oủ elles s'unissent avec les cellules. Ces commissures jouent le rôle de muscles transversaux. - Chez I Ascaris mucronata le la tote, les muscles longitudinaux ne sont plus formés par des cellules distinctes, mais par des faisceaux de fibres excessivement fines (PI. IIIl, fig. 13). Les faisceaux eux-mêmes sont larges d'environ $0^{\mathrm{mm}}, 016$, et l'on reconnaît facilement qu'ils sont issus génétiquement de cellules semblables à celles de l'Asearide du triton, car on retrouve çi et lì, à leur surface, les gros nucléns (Fig. 19, a) munis d'm ou de plusieurs nucléoles. Cés faisceaux sont unis ensemble par des commissures nombreuses. Si l'on compare notre dessin arec ceux que Meissner donne dn système nerveux périphérique des gordiacés, on reconnaitra quils concordent parfaitement. Cependant chez notre ascaride nous n'avons point affaire avec des nerfs, car on peut poursuivre facilement une des commissures et reconnaitre comment après être issue d'un faisceau 
de fibres longitudinales elle va se perdie dans un autre. De cette ascaris de la lote il n'y a qu'un pas jusqu à l'ascaris du chat (A. Instax), chez laquelle de larges commissures unissent les muscles longiludinaux, et sont si évidemment de nature musculaire gu’il ne viendrait à l'esprit de personne d'en faire des nerfs. La partie postérieure de l'Ascaris mucronula contient du reste encore des muscles composés de simples cellules. Tels sont, par exemple, le muscle rétracteur de l'anus (Pl. V], fig. 2, g) el les muscles flédrisseurs de la queue (Pl. Vl, fig. 1 e, et fig. 2 e). Ces muscles sont munis de gros nucléns renfermant en genéral un grand nombre de nucléoles. Ces deruiers se dissolvent rapidement dans l'acide acétique. Chez le mâle. on trouve une cellule musculaire étoilée (PI. VI, fig. ‘) suspendne librement dans la cavité du corps en arrière de l'anus. Elle émet des prolongements qui vont s'attacher les uns (Fig. 4, a, a' a") á la paroi dorsale du corps, les andres i la paroi ventralc (b) et i l'anus (c).

Nons navons fu trourer ui le système nerreux ni le système circulatoire lécrit par Blanchard chez les Nématodes. Le seul organe yu on puisse considérer, a notre aris, comme de nature nerreuse, c'est l'accumulation de cellules verdâtres que l'on trouve sur la paroi ventrale de charue còté de la lig̣ne médiane chez certaines ascarides (A. suilla).

Les organes décrits par Meissner chez les Gordiacés sous le nom de hoyaux à cellules (Zellenschläuche) paraissent être identiques avec les rubans intermusculaires qu'on trouve chez les r'ais nématodes ${ }^{1}$. Chez quelques-uns, il est vrai, nous n’avons pas constaté l'existence de cellules dans leur' intérieur (A. suillu, A. Mystax), mais dans l'immense majorité des espéces nous les trouvons constitnés à peu près comme clıez les gordiacés. Tantôt ils renferment des cellules munies réguliẻrement d'un seul gros nucléus (Cucullenus elcyens, Ascaride du triton, de.): tantôt les cellules de la plus grande partie da boyan ne renferment igalement qu'un senl nucléus, tandis que celles qui avoisinent l'extrémilé postérieure du corps en renferment un gran! nombre. C'est le cas chez l'Ascuris mucronuta de la lote.- Gabriel a dicrit les boyaux à cellules chez le Cucullames cleyums comme trois glandes découvertes par lui (a me detectis), qui s'ouvent à l'extrémité postéricure du corps i l'aide de trois prapilles. A cela il y a a objecter: I" que les papilles ne se trourent que chez les femelles, tandis que les boyanx il cellules existent chez les deux sexes; $q_{0}$ yue le nombre des papilles n’est point constant et quion trouve a peu près autant d'individus yui n'en ont que denx, une ou point, que de reux qui en ont trois:

1 Il es du reste raisemblable à nos yeun qu'il n'y a ancune raison pour séparer les fiordiacés des Némalodes. 
$3^{\circ}$ que les papilles ne sont point percées d'un canal; $4^{\circ}$ qu'il y a quatre boyaux el uon pas trois. Il est par suite évident pour nous que les boyaux ne s'ouvrent pas it l'extéricur, du moins à cette place-li.

Nous arons encore it mentionner ici des organes particuliers qui paraissent se retrouver chez divers nématodes et qu'on pourrait pentêttre considérer comme des glandes anales. Ce sont de grosses cellules nuclées et nueléolées qni entourent le rectum et qui sícrètent peut-ềre une substance destinée à être déversée dans l'intestin. Chez l'Asruris mucronatit ces cellules sont an nombre de trois on quatre (PI. Vl, fig. I a, et fig. a) a), et atteignent une grandeur de $0^{\text {mm }}, 09$. Che $\%$ une asearide de la grenouille rousse (Ruma lemporteria) pue nous décrirons plus loin, elles sont un peu plus petites et plus nombreuses.

Nous anrions voulu encore parler des organes singuliers ipui tapissent la paroi interne du corps chez l'lscaris suillu, organes qui, il notre connaissance, n'ont pas dé dẻcrits jusquici. Mais nous ne voulons pas itendre plus Ioin cette digression déja si lonģue.

\section{DE LA FORIIITION DES OEUFS.}

On peut des l'abord distingner chez les nématodes deux catégories d'especes par rappol't an mode de formation des aufs. Dans l'une, les aeufs sont groupés, lout au moins dans le vilellogène, autour d'un rachis central. Chez l'autre, il n’y a pas trace de rachis. Afin de mieux délimiler ces catégories, on peut dire que la premirre renferme les especes chez lesquelles le vilellogène contient plusiens wul's dans une mème section transversale, tandis que la seconde est forméc par les espèces cliez lesquelles le diamètre dı vitellogène est occupé en général par un seul out. En effet, il est probable que daus le premier des deux cas il existe foujours mu rachis on son équivalent.

Nous cousidérerons d’abord la formalion des anfs chez les espèces ippartenant ì la première calígoric. C'est ici que vient se ranger l'dscaris Myslax ì jamils célèbre par les débats qu'elle a suscités. A ses côlés se place l'Ascuris suilla. Nous nous ocenperons de préférence de 
celle dernière, parce qu’elle est plus propre à servir de type amx Ascarides dont les auls sont groupés aulour d'un rachis. Ce dernier est en elled chez elle d'un tiamètre beancoup plus considérable que chez l'Ascaris Mystux ${ }^{1}$, et peul être distingué immédiatement sans préparalion à lravers les parois de l'oryane, comme une colonne de couleur noire placéc daus l'axe lu vitellogène cylindrique.

La première question qui se présente est celle relative aux premiers ćléments résiculeux que l'on rencontre daus la partie aveugle de l'ovaire. Arous-nous affaire lì à des résicules germinatives, on bien à des auls véritables, ou enlin ì des cellules-mires les reufs telles que les weibliche Keimzellen de Meissnei?

Nous te dirons rlis l'abord, nous nous joignons ì Nelson, Bischoff et Thompson pour recomnailie dans ces éléments vésiculeux les vésicules germinatives des aufs qui vont se former. Il est possible que le schéma de formation des wuts lécril par Meissner, d'abord chez les Mermis, existe réellement chez les Gordiacés, mais nuus contestons son exactimle prour lous les vais némilodes que nous avons observés. D'après la description de Meissuer, on devait rencontrer, dans la parlic de lovain qui est connue sous le nom de blastogène, des collules dont les nucléns se multiplient par une sorte de génération endogìne. Or, les nucléus (vésicules germinatives) existant seuls ì ce moment, nous n'avous jamais réussi ì trouver aucune de ces cellules-germes (Keimzellen) munic de plusiems nucléus.

Quant à la maniere dont s'engentrent les vésicules germinatives dans le blistogine, nous ne pouvons rien dire de particulier. Nelsour ' décrit la surface interne de la membrane le l'or'gane comme semée de granules qui se détachent. Ces granules sont destinés, d’après lui, à devenir les taches germinatives des auls. L'élément primaire de l'enuf serail, par suite, la tache germinative, comme kö̈liker l'avail

'Le rachis est néanmoins évident chez l'Ascaride du chal, nous ne sarons nous expliffuer comment Reichert a pu en nier complétement l'existence. It. Reichert's Jahresbericht, 18:3, in Vlïller's Arehiv für Analumie und Physiologie.

Loc. cit., p. 572 . 
déji almis. Malheureusement nous nirons pas léussi à nous convaincre de lexactilude de celle observation. Nous n'avons jamais rien vu que mous pussions avec quelque váasemblance considérer comme mu tache eneminative libre, non entore entourée te sa vésicule. Il ne nous semble pas improbable que les vésicules germinatives se multiplient dipetenent soil par division, soit pentètre par génération cndogène. Mais nos eflonts pour observer quelque clıose de semblalule oul été aussi infinchurux que cenx de Thompson.

Le baslogene soil chez I'A. Mystax, soil chez l'A. suilla, est de linit uris-cont el se continue dans la parlic lu tube génératem qui a regu le nom de vitellogène. Il est fort difficile de dire on le llastugène finit el où le vitellogène commence. On peut, il as vai, admetue comme point d’orignine de ce dernier la place ou les parois du lube générateu. commencent à moutrer il leur surfice interne les côts longimbinales granuleuses, par lesquelles Nelson el Bischoff font sécréler lo vitellıs. Mais cette place elle-méme n’est par lacile ì déterminer, parce que les stries gramuleuses commencent comme une trace imperceptible fort dilficile à poursuive. L'origine du vitellogène se tronve de fail lì, on - une substance se dépose pour la premnipre fois mutre les vésicules germinalives, substance qui sera appelée à lare partie intrinsèque llu vitellus. Or, i de point de vate, la distinction du blastorène et du vitellogène noms parait tont an phs justifiée. Déjì dans le soi-elisant haslogine les résicules germinatives sont agrghtinées ensemble par une sulstince transparente intercellulaire, ou, si l'on aime mieux, intermucléaire. La masse de cette substance s'augmente ì mesme que les résicules descentent daus l'oraire; il se forme les granules dans son intérieur, et l'on a le premier rudiment du vitellus.

La partie supéricuredu vitellogine proprement dit est remplie par me colonne à apparence gramulense, dans l'intérieur de laquelle on a déji beanconp de peine ì reconmaille l'existence des résicules germinatives. D'un autre côté, la séparation des oufs les uns lles antres an moyen d'une division de la substance vitellaire n'al pas encore en lieu, de sorte que lá colonne a, en apjarence, une consistance assez homogène. Bientôt 
cependant on voit des granules plus gros que les autres s'ordomner en ligne snivant l'axe de la colonne (Pl. III, fig. 1, a). Cette ligne, encore fréquemment interrompue, est le premier indice du rachis central. Plus has (nous ne parlons ici que de l'Ascaris suilla) Je rachis se montre comme un axe noir ef épais all centre de lit colomne (Pl. III, fig. 2, d). La surface externe le celle-ci est mamelonnée (Fig. 2, c). Chaque mamelon répond à un cuf en train de se former et renterme dans son intérieur une résicule germinative. Lorsqu’on déchire la colonne avec des aignilles, on reconnaìl pue les ouf's sont à ce moment des pyramides allongées (PI. III, fig. 5), à base convexe. Les bases sont dirigées vers la périphérie el forment los mamelons que nous venons de dícrire; les sommets convergent tous vers le rachis. Meissner, qui a ohservi le rachis chez l'A. Mystax, espece chez laquelle il est beaucoup moins évident que chez l'Ascaris suilla, déclare quil n’existe qu’en apprarence ol qu’il es formé par les restes des cellules-mères des aufs, superposées les mes aux aures. On serait tenté de croire que Meissner n’a sontemu celle ophinion yue par amour pour la théorie, s'il n'avait pas flonne hi-meme une entorse grave à cette dernicre en recomnaissant que cerlains nématorles, comme le Strongylus armatus, possedent m rachis réritable an lieu d'un rachis apparent. On comprend difficilement que la connaissance de ce finit n'ail pis sonlevé des doules dans l'esprit dr Meissner sur l'exachilude el la justesse de sal lhéorie de la formation des aul's. La figure qu’il dome ${ }^{1}$ des ouf's du Strongylus crmulus alhérents à leur achis, représente beaucoup mienx le véritahe étal des choses chez l'Ascuris suilla el l'A. Mystax que sal longue deseription si riche en détails. Tous les némalodes qui ont plus d'me simple rangé d'unfs longitudinale dans l'ovaire se rangeront sins ancun donte atu type de ce strongyle, et pour ce qui nous concerne nous ne considérons point comme improbable que les grordiacés doivent prendre un jour le mèmr chemin, bien que ce ne soit lì qu'me pure supposition. 
Lorsque les aruls ont alleint une cerlaine grosseur, on peul, en passant avec précaution arec des aignilles sur la colonme d'eut's sortie du vitellogène, détacher la plus grande prarlie des wuls du rachis, de manière à obtenir ales fiagments de colni-ci analognes a colni yne nous avous figuré (PI. III, fịc. 4). On recommait alors que le rachis forme

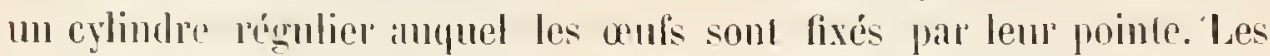
granules vitellins remplissent anssi hien le rachis que les ant's enxmèmes. Ils soul mème an beancoup plus grande abondance dans le premier que dans les seconds. Do pareilles préparalions ne laissent ancun doute sur la conformation du vilollogine. Il es vrai qu'en léchirant la colonme doufs aver des aiguilles daus la partic inférieure du vitellogèue, on ohlient en grand nombre des groupes d'renfs en forme d'éloile (Pl. III, fig. ä) analogues i ceux que Meissner a figurés chez

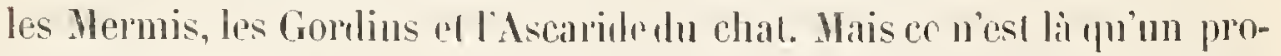
dnil artificiel. I! est évilent que des qu'on déchire to radhis en pelits fragments, les auls encore alliérents par leur pointe ì ces liragments doivent paraitre distribućs en groupes biloilés. Meissner a voulu voir dans ces groupes des neufs à péloncule creux, encore unis ì une cellule-mère placée an centre. Colle soi-disant cellule-mère n'est quiun fragment ilu rachis.

Nelson et Thompson ne disent mot du rachis a propos du vitellogine de l'Ascaris Mystax. Ils se contentent de dicrire les oufs comme allongés en pointr ol pressés les uns contre les antres. Les pointes élant loutes dirigées vers le centre, il en résulte que les ouls prennent une forme pyrmidale. Le rachis nest point, en elfel, aussi facile ì reconnailre chez I'Ascaris Mystux que chez l'Ascaris suilla. Il est heancoup moins large el par suite il se déchire avee une grande facilité dans la préparation. C'est e qui explique pourquoi l'on a beamcomp plus de peine à obtenir chez celle esperce que chez l'Ascaris sulla de longs lirayments du rachis léponillés d'une grande partie dr lours aufs. En revanche, on obtient de nouvean chez colle ascaride, par la préparalion avec rles aignilles, un grand nombre de groupes d'oufs étoilés. C'est li ume preuve que les anfs sont hien allhérents an centre el forment un rachis 
dans l'axp du vitellogène, mais qu'ils ne sont pas simplement pressés les uns contre les autres, ainsi que Nelson, Reichert et Thompson paraissent le croire. La difficulté qu’on rencontre à préplarer de longs fragments du rachis el la facilité arec laquelle celui-ci so déchire, chez l'Ascuris Mystax, en petiles parcelles portant me conrome d'aufs ì lem' pourtour, expliquent la méprise de Meissner el sa llúorie erronée sur la formation des arufs.

Une question qui a suscité un débal assez vil" entre Bischofl et Meissner, est relle ve silvoir si les reufs dans le vitellogène sont mumis l'une membrane enveloppante on non. Si la théorie de Meissner sur la formation des andés étail exacte, ces derniers derraient posséder évilemment une membrane vitelline. Nous avons vn que celte throrie priche par sa base doit être rejetíe. Mais il ne suit point encore de là avec nécessilé que les aul's soient dépourvus de membrane, comme le pensent Nelson, Bischoff et Thompson.

La question rest de lait difficile, d’autant plus difticile mìme quä notre avis elle est assez oiscuse. Avaut de discuter avec ardeur l'existence ou la non existence d'une membrame, il serait bon de s'entendre sur ce qu’on veul désigner par ce terme. C'est lí mme pensée qui naquit dans notre esprit ì la lecture d'um passage d'Allen Thompson. Le savant écossais ' lit en effet qu’il n'est jamais parvenu i reconnaître chez les oufs, aussi longtemps que ceux-ci séjoument lans la partic le l'ovaire où le vitellus se forme, la moindre structure qui cit pu lui faire admettre l'existence l'une membrame enveloppante. 'Tout in contraire, la surface des aufs lui a toujours semblé couslituée comme colle d'un protée. Or, la question n'est point tranchée par lí; lo délat se trouve simplement transplanté sur un antre terrain. Il n’y a pass de question plus controverséc que celle de sivoir si les protées (amcebas) sont limités par unc membrane ou non. Il n'y a pas Jongtemps qu'Anerbach ${ }^{2}$, dans un travail étendu sur l’unicellularié des Amokis, s’ost prononcé

\footnotetext{
${ }^{1}$ Loe. cil., p. 4:33.

${ }^{2}$ Ueber die Einzelligheit der Amwen. - Zeilschrifl f. wiss. Zoologie, I85a.
} 
pour la première alternalive. Si donc Anerbach a raison dims sa manière de voir, il s'ensuit lorcément que la remarpue de Thompson, an lien de servir à justifier l'opinion de Nelson, doit an contraire, sans que son auteur sen donte, parter en fireur de Meissner.

Une menthrane est me conche mince d'une substance, dont la composition chimique ou lien les propriétés physiques (ténacité, densitë, elc.) sont diflërentes de relles des sulıstimces qui limitent relte conche te part el d'antre. La surface d'un imoba est très-probablement formée par me conche plus dense que le reste du corps de l'animal. Mais il n'est pas impossible que la densité du corps de l'amoulba aille en se modifiant par degrés de la périphérie vers la limitr de la cavilé du corjes, à peu prés comme la densité du cristallin va se modifiant insensiblement de la périplérie vors le centre, mais dans un sens inverso (li partic la plus dense du eristallin est, comme l'on sait, la parlie centrate). Dans ce cas, on ne peut pas parler de membrane proprement dite. Le corps est bien limité par mne couche plus dense, mais cette couche ne mérile pas le nom de membrane, parce que sa limite interne est indéterminće.

Ilngo von Mohl a léjà en soin de fiaire cette distinction dams le règne régrital. Il sépare des membranes sous le nom de pellicules ces couches qui ne sont point neltement délimitées d'un côté, mais se foudent avec la substance roisine. Ia distinction est juste, mais le nom de pellicule n'est peut-être pas tris-heurensement cloisi.

Les renfs de l'Ascaris Mystax se comportent, ì notre avis, d'une manière parfailement semhlable. Dans claque ouf il y a deux choses i distingner : d'abord les gramules vitellins qui, vus an microscope, offrent une couleur sombre, paree quils réfractent fortement la lumire, puis me substance transparente, glutinense, qui sert à rémir les granules entre ex. La partic périphérique de l'œuf est forméc uniquement par cette substance transparente intergranulaire; l'ceuf parail en conséquence entouré d'une zone claire tris mince dont Meissner a lait sa membrane vitelline. Si les granules vitellins ne pénètrent pas dans cette conche périphérique de sulstince interọranulaire, c’est parce que 
cette substance est plus densi dans cette région que dans le reste du vitellus.

Tout le monde est d'accord pour recomnaitre l'existence d'une mentbrane antour de l'oul' dans l'ovidnete, membrane qui ne se lorme, d'après Nolson, Bischoff et Thompson, que depuis que l'ouf est fécondé. La formation de celte membrane n'est, m lont cas, pas en relation directe avec l'acte de la fécondation, car olle a lieu aussi bien chez les f'emelles qui n’ont pas été lécondées que chez celles qui l'ont été. Il n’est de firit pas possible de spécilier dans quel noment celle membrane apparaî, comme cela se comprend de soi-mème. En effet, ce u'est point li une produchon complétement nouvelle. Elle est formée par la conche phos dense de la sulsstance intergranulaire du vitellus, conche qui va se diflérenciant loujours davantage du reste de l'oul', el qui, acquérant une densité tonjours plus considérable, arrive à se délimiter par une ligne tranchée du còté du vilellus. Ia conche périphérique plus dense du vitcllus se transtorme peu à peu en me vaaie membranc. C'est pour cela que nous arons taxé d’oiseuse la dispute yui s'est élevie an sujet de la membrane ritelline entre Meissner et Bischofi. De fail, les deux parties ont raison. Bischoft a le droil de nier lexistence de la membrane vitelline dans le vilellogine, puisque cette memhrane n'est pas encore diférenciće comme une nembrane incontestahle. D'un antre côté, Meissner pent jusqu'ì m certain point sontenin l'existence de la membrame, puisque celle-ci est déji virtuellement à sa place, étant en chemin de se forner, de se différencier.

Il y a deux opinions en présence quant à la manière dont les granules vitellins sont engendrés. D’une part, Meissner admet quils sont sécrétés par les parois des cellules-mères, et quils passent de lintérieur de celles-ci au travers des pédicelles ereux jusque dans les reufs. Cette manière de voir u’a pas besoin d'être combaltue, puisque nous nions l'existunce de ces cellules-mères. D'antre part, Nelson et Bischoff font sécréler ces granules par les còtes gramuleuses longitudinales et légèrement contonmées en spirale, dont est munie la paroi du vitellogìne. Thompson, plus prudent, laisse hien les geramules se téposer à l'extí- 
rienr, tont antour, des résicules gemninatives, comme Nolson et Bischoff, mais il préfère ne pas se prononcer sur l'origine de ces granules. Sa description est, dans tous les cas, parfaitement exacte jour la partie supérieure du vitellogène où le rachis n’est pas encore apparent. Là les vésicules germinatives sont aggolomérées an moyen de la sulsstance gramulitre tramsparente ef les gramules commencent à se montrer d'abord en fort petil nombre tout antom' de ces vésicules. Plus loin, l’étal des choses est un peu différent. Le rachis est formé, el sur fout son pourtour sont fixés par leur pointe les aufs à forme pyramidale. Si les gramules vitellius étaient sécrétés, commè Nelson et Bischoff le reulent, par les parois du vitellog̨ine, il fandrail que ces granules se déposassent sur' le pourtom des oufs. Mais ce pourtour en est an contraire toujours exempt; il est formé par la couche plus dense de substance interorgranulaire. D'ailleurs, on ne renconire pas de gramules vitellins lihres entre la paroi du vitellogène et les auls, ce qui derrait aroir lieu si l'organe sécréteur des granules était logé dans la paroi elle-mème. Il est une circonstance qu’il ne linut pas perdre de vue, c'est qu’ì l'époque où les auls sont encore petits el ou la vésicule germinative est encore facile ì recommaitre, c'est-i-dire à l'époque on le ritellus ne contient encore que peu de gramules, le rachis est au contraire tris-richement pourro de granules vitellins (Pl. III, fig. 4). Les granules du rachis sont alor's en contact immédiat avec ceux des aufs. A ce moment le rachis a un diamitre issez considérable. Lorsqu'on descend dans les réggions infériemes du vitellogène, on s’aperçoil que les aufs grossissent par dlog̣és; Jes granules vitellus deviennent tris-abondants dans Jeur intérieur; anssi les oufs perdent lenr transparence el il n'est bientót plus possible de reconnaitre la vésicule germinative, perdue dans la masse du vitelhus. Celte vésicule existe cependant bien toujours, car sons l'action de la teinture d'iode très-ćtendue, elle prent une teinte brume beaucoup plus intense que le vitellus, ce qui permet de la reconnaìtre aisément. Dans cet état il arrive sourent que les aeufs deviennent irréguliers il la périphérie. Il se forme ì leur surface de profonds sillons qui semblent les diviser en plusiemrs lobes (PI. III, fig. כ̌, a). Tandis 
que les oufs se développent ainsi el croissent dans toutes les dimensions, le rachis s’atrophie, devient plus étroit el finit par disparaître complétement. Les granules qu’il contenait ont passé dans les ounls. Des lors il ne nous semble pas improbable que les granules vitellins qu'on trouve dans les uufs proviennent tous du rachis. Ils se loment dans ce dernier el passent an travers des pédoneules jusque dans les aenfs. Celle maniere de voir se rapproche de celle qua soulenue Meissner. Jeissner faisait naîtue comme nous les granules vilellins dans l'axe du vilellogène. Lioryane qui les sécrète devail se trourer, suivant lui, dans les éléments du rachis, dans ses cellules-mères ou cellules-germes (lieimzellen). Nous rejelons l'existence des cellules-germes, mais nous crogons devoir conserver au rachis lui-même la lonction d'organe préparalenr des gramules vitellins. Nons ne ponvons aller plus loin dims notre investigation et dire de quelle maniere ces gramules se forment dans le rachis. C'est ici que l'observation cesse et que l'hypolhrise commence. Il est probable que les parois du vilelloğène sćcrètent le liquide qui remplit cel organe el qui baigne la colomme lormée par le rachis portant les auls sur son pourtour. Le rachis absorbe sans doute ce liquide, el c'est là le matériel aux dópens duquel il forme les granules vilellins.

Comme nons le disions, le rachis s'atrophie par degrés dams la pantie inféricure du vitellogène et finit par disparaître complétement. Les aufs se séparent les uns des autres, tout en conservant pour m temps encore leur ancien arrangement. Les pointes convergent toujours vers l'axe du vitellogine; elles se touchent, mais sills adlórer los umes aux ilutres. L'aul a encore à ce moment sil formo pyramidale, et cest le sommet de la pyramide, le proint où l'ouf s’est délaché du rachis que Nelson à désigné sous le nom de broken edye el que Meissner considire comme une véritable ouverture, comme un micropyle. L'existence ou lit non existence de ec micropyle est mme question capilale, puisque Meissner fomde sur celte ouverture toute l'explication qu'il dome du phénomène de la fécondalion. Celte question semble de noureau se réduire à celle de l'existence on de l'absence de la membrane vitelline. 
Nelson, Bischufl et Thompson, qui nient la membrame, ne reulent piats non phus entendre parter du micropyle. On concoit eependant la jossibilité de l'existence d'un micropyle, mème dans le cas où l’ouf n’est pas enveloppé par tne rarie membrane, mais où la périphérie du vitellus a simplement acyuis me consistance plus dense que le reste. Une déchirure le cette conche plus dense prodnit me véribale ouverture de lis périphérie, et lon comprend aisément qu’m corps étranger puisse pénétrer plus facilement daus l'oeuf à la place oì cette conche plus dense manque que partout ailleurs. Mais il ne nous est pas possible d’admothe l'existente d'un micropyle, mème dans ce sens-là, chez les aut's de l'Ascaris du porr ni de celle du chal. Les exuls pyramidaux ne se détachent pas dı rachis par une véritable déchirure, mais la pointe ou pédoncule de claque urul" se resserre graduellement it son point l’attache. I.e lien d’alhésion levient toujours plus ét’oit, linit pas être pour ainsi dire un point mathématique, après puoi la séparation a lien. Celte séparation ne laisse par suite ancune ourerture ì la surface le l'ueuf; la place nag̨uère allıérente est recouverte par cette même conche transparente et plus dense qui se trouve sur loute la périphéric. Ide. micropyle n’existe donc pas.

Les oul's arrivés dans l'oviduete thangent peu ì peu de forme. Ils se. contractent, leur pointe s'émousse; en un mot, ils s'arondissent par degrés. A ce moment-lit, dit Meissmer, le micropyle devient difficile it reconnaitre. Nous le croyons sans peine. - C'cst aussi a ce moment que les oufs arrivent en contact avec les zoospermes chez les femelles qui ont été fécomlées, el que la fécondation les oufs s'oprire. Nous traiterons ce sujet en détail dans un autre clapitre et nous renroyons ì ce moment-lì lá tìche de poursuire les modifications que subissent les aufs chez les femelles yui n’ont pas été fécondées. En effet, nous tronverons dans ces modifications la clef de phusicurs des erreurs qui se sont glisscés dans les diverses appréciations qui ont été faites de la lécondation chez les nématodes.

Il est sans doute beaucoup d'ascarides tont les auf's se développent de la même manière que ceux des deux espèces qui ont fait le sujet des 
lignes qui précident. Siebold eite l'Ascaris aucta, l'A. lumbricoüdes, l'A. osculata el le Strongylus inflexus comme possédant un rachis daus l'ovaire. Les observations de Meissner nous ont également appris l'existence d'mu rachis chez livers Gordiacés, ainsi que elıez l'A. marginata, A. megalocephala, li Filariu mustelarum et le Strongyhus armatus. Or, nous considérons comme lor't probable que chez toutes ces espèces munies de rachis les aenl's se développent identiquement de même que chez les Ascaris smilla of Mystax.

Aucuu des individus de l'Ascaris mucromata de la lote que nous avons olsepvés n'avail alleint sa maturite sexuelle, soit parce que la saison nélait pas favorable, soil peut-iàte anssi parce que ce nématode n'est pas appelí à alleindre sa maturité dans ce poisson. Le tube générateur des lemelles élait méanmoins toujours rempli d'ovules dont le diamètre ne thépassait pas $0^{m m}, 006$ ì 0,007. Ces ovules élaient groupés autour l'mu ance central dams le vitollogène. Soit l'axe, soit les ovules enx-mèmes étaient incolores, te sorte qu’il nétait pas très-facile de constater l'existence d'un lakluis. Cependant, Jor'sque nous essayions de séparem les ovules les uns des autres par mue légère pression, nous les voyions

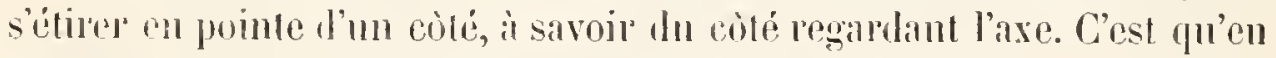
effet là se trouvail un rachis très-délicat.

Lit partie arengle de l'orairre est remplie chez l'Ascaris mucromata pal'

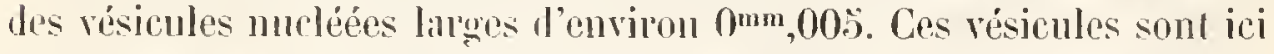
de nonreau les vésicules germinalives arec leur lache caracléristique, el il pranit certain que chez tous les nématodes la vésicule germinative est l'élémunt primarire de l'oent. Le blaslogìne n’ayant chez l'Ascaris mucronata qu'me largent denviron $0^{\mathrm{mm}}, 01 \mathrm{~s}$, ne pent comprendre plus de deux vésicules germiutives dans si largeur. Ces vésicules s'entourent d'une mince couche d'mne substunce ghlutineuse et ineolore. C'est là le premier rudiment du vitellus. Nous n'avous pas rencontré d'individus chez lesquels les mufs arssent atteint un développement phus considérable.

Dans l'intestin du Tritom temiatus nous avous rencontré assez fréquemment une ascarille dont nous arons déjà décrit ailleurs les muscles. 
Nous ne la trouvons mentionnće ni dans Dujardin ni dans Diesingy. Les miles paraissent être beaucoup plus rares que les lemelles, comme c'est te cas chez lant de némilodes, du moins n'avons-nous rencontrí que des femelles. L'amimal enlior est fort grèle, il alteignait me longrleur d'envidon 7 i 8 millimèlres. Lomverture du vagin est siluće entre le second et le troisiome liers de la longueur. Celle ascanide est munir comme l'Ascaris mucronula dant d'antres d'un organe glanduleux qui s'ouve à lextérieur dans la parlie antérieure du ver, sur la fare ventrale. Elle vaattegnait jomais la maturié sexuelle dans les conditions où nous l'avons rencontréc. Cependant l'ovaire élail rempli d’ovnles.

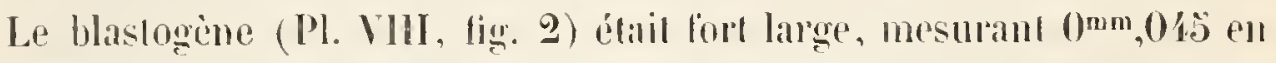
diametre. Il était plein de résicules geminatires langes d'muiron

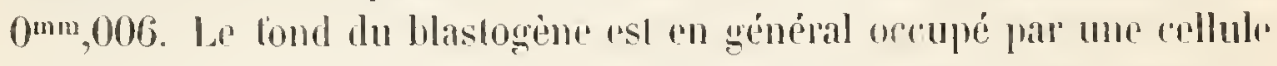
ovale, trois fois aussi longur que las vésicules germinatives et munir d'un gros nucléns. Malhoureusement il ne nous rs pas possible de dire si cotte collulo est dans un lappont génélique quelcongur avec les vésicules germinatives. Ces depnieres sentoment d'um rudiment de vitellus, précisément romme chez l'Ascaris mucromete do la lote.

Parmi les nématorles dont les auls sont groupés autour d'un lachis dans le vitellogìne, nous mentionmerons oncore le Cucullamus elegans dr l’intestin des poissons d'rau douce. Siebold cite' dejà co ver an nombre des nématodes elrez lesquels il a constaté l'existence d'un rachis. Il es à regretter qu'il ne soil entré dans aucun délail à ce sujel, can to rachis du Cucullames elegaus n'est point facile it recommaitre, et les deux seuls auteurs qui se sont occupes particulierement the la formation des ouls chez cel animal, i savoir liölliker ef Gabriel, nont su le voir ni l'un ni l'autre?

D'apris Gabriel, les oufs se montrent d'abord sous la forme de simples risicules : les résicules gemminatives. Celles-ci sont, d'après lui,

\footnotetext{
'Handhuch der vergleichenden Anatumie, 1, p. 121. Anmerkung 2.

2De Cucullani elegantis evolutione. Disser. inaus. Berolini MoCCCLIH, Auclore Benun Gabricl. p. $\ddot{3}$.
} 
dh́pourvues de tonte espèce de nucléus, car le nucléus u’aparaît, dit-il, que dans le liers inférien du tube ovarique on il est engendré par voie pndogìne. Bagge avait, il est vai, dija décrit ol figuré la lache germinalive dims les vésicules lu blastogène chez d'autres némalodes (Strongylus auricnlaris of Ascaris acuminala). Nais Gabricl ${ }^{2}$ léclare que la lache vur par Bagege n'étail qu'me formation fortuite, protuite fiur me pression due à la plaque de verre dont átail recouvert l'objel (Arbitror autem hane maculam tantum fortuitam formationem fuisse productam pressione quadam in rem observatam exercila). Nónmoins l'observation de Bagge est parfintement exacte pour ee rui roncerne le Strongylus amricularis el l'Ascaris acuminata. La description de cet inteur pent môme stre ćlendur, malgré les ilonmées de Gabriel, an Cucullamus elegans hui-même. Nous avons examnion me quarantaine de Cucullans femelles, et dans chacm nous avons trouve le blaslogine rempli de cellules molétes, r'est-i-dirr de vésicules germinatives munies de lemr tache de Wagner. Kölliker fait naitre l’abord dims le blastogino les taches germinalives, de la même manière que Nelson l'a représenté plus lard chez l'Ascaris Mystax. Pour re yni nous conceme, nous n'arons rien vu que nous enssions pu interpriter avee vaisemblance comme des laches germinatives encore libres; nous avons an contraire tronvé les résicules germinatives jusqu’an fond du blastogène.

II est encorr moins loisible chez Ir Cucullanus elegans que chez les repéces que nous avons consilérées jusqu’ici de tirer mo ligne de démarcation positive entre le blastogène of le vitellogène. Le vilellogène s'ótend de fail jusqu'an fond de l'extrémité aveugle du lube sexnel. En effor, les vésicules gemninatives sont séparies les mes des antres par me substance transparente, qui est le premier rudiment du vitellns. On peut même recommaitre déjà dans la partie avengle de l'ovaire des lignes très-déliées qui indiquent le contom des ovules (PI. IV, fig. 1).

\footnotetext{
1 Joc. cit., p. 8.

2 Joe. cil., |, 5 .

${ }^{3}$ Loe cil., 1. 7?
} 
Cenx-ci sont pressís les mos contle les antres de manive à prendre une forme polyédlique, of ils allòent les uns anx antres an point de ne pouvoir ètre fireilement isolés. I ses vésicules germinatives onl dims

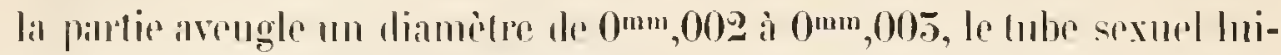

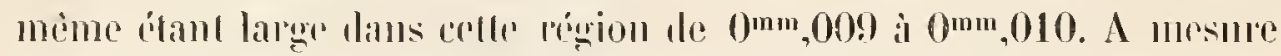
que les aufs deseculdent daus l'ovaibe ils croissent en dimensions. La vésicule germinalive elle-méme angemente rapidement de diamidre. -

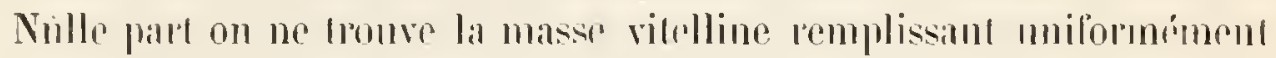
lo lube sexmel, avee les vésicules germinalives dispersios dims son intériem, bien que Gabriel ail merresenté les choses ansi. Parlont, an

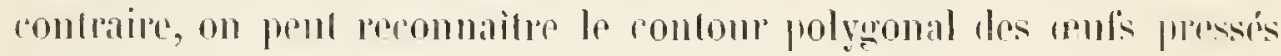
les ums contre les antres.

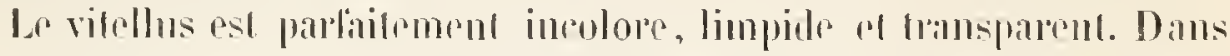
son interient on ne recombit quin fort pelit nomber de minimes gra-

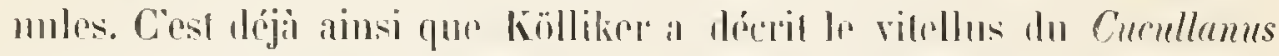
elegans. Cependant Gabriol est venu contester l'exaldude des domóes

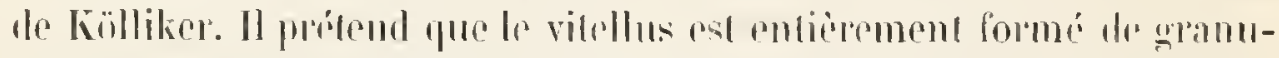

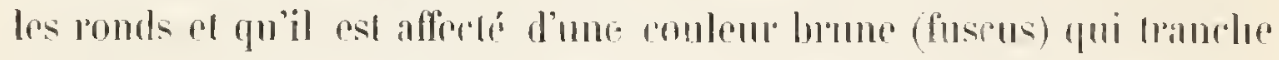
sur la membrame incolore enveloppante. Il as posible que la saison ou replaines antres circonslances plus on moinsmormales intluent puol-

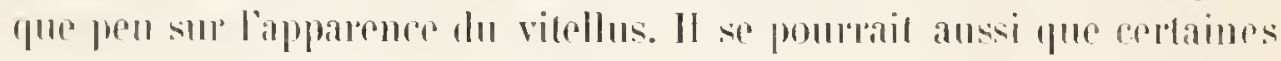
différences dépendissent de l'pspiere de poisson habilép par lo Cuenllan. Cependant tous les individus que noss arons examinés provenaienl de

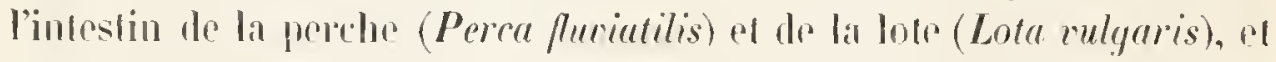
Gabriel parail avoir anssi pris poun objed de ses observalions des farasiles de la perehe. Nous ne silvons done trop comment explifpuet dedlo différence dans les résultals. Nons alvons loujours lromve les dommes de

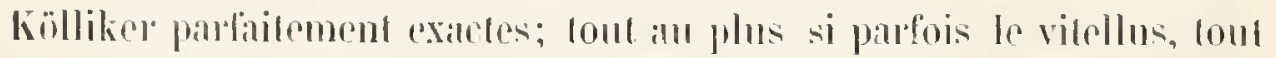

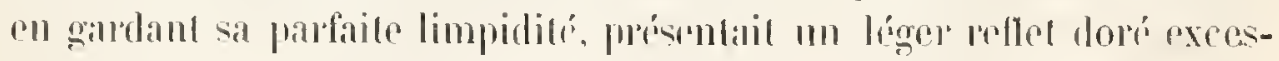

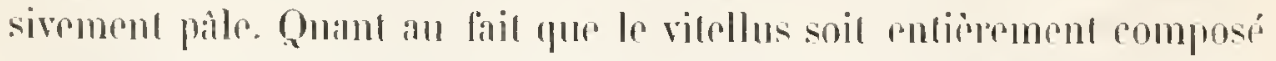
dregramules, nous davons dire que la phupart des réaclifs chimirgues font prende cheffet à la substance vitelline ume consistance gramuleuse. Mars nous ne samrions dire si ces réactifs nout dautre effot que de faime. 
micux ressortir une structure próxistante, ou bion s'its cansent un précipilé chimique dans le liquide vitellin. Tonles hes fo is quon obsenre les ceufs dn Cucullamus elegans dans de l'ean pure, on mêno dans une dissolution saline pen concentrér (atin drompecher la dilluence), le vitetlus so montre clair et transparent, presqua sans garanules.

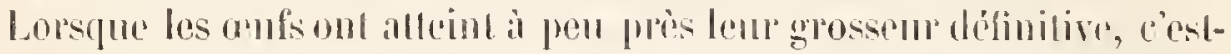
i-dire colle quits ont an moment de la fécondalion, ils sont pho faciles a séparer les uns des andres. On reconnaîl alops quiks sont exactement

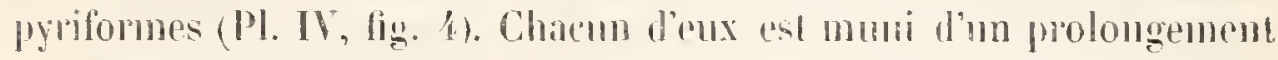
romparable an pédicelle llum poire. Lus aufs sont disposés dans le

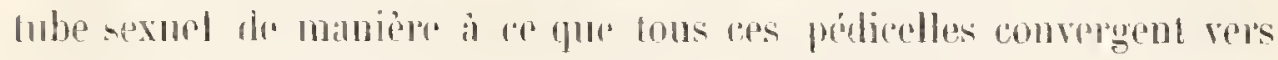

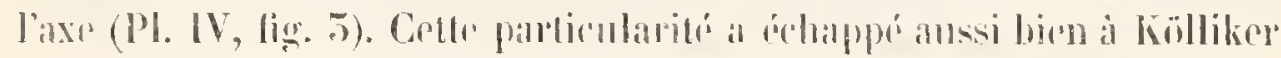

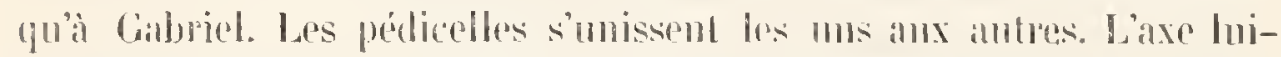
mème est acenpé par nn bilament délié, duqued bes pédicelles des auls partent comme aulant do rameanx. Corst la un lakchis fort délical. Lors-

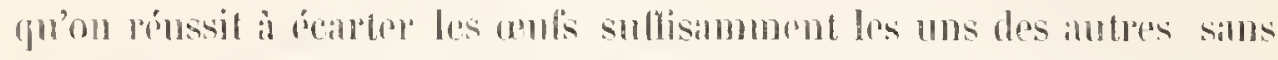

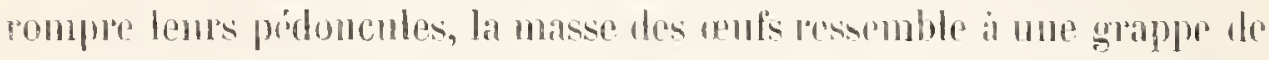
raisin, dont los ramilicantions seraicnt excessiventent gretes par rapport

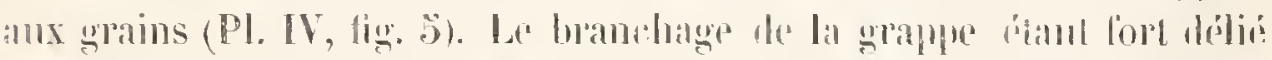

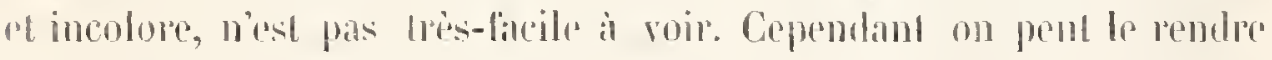

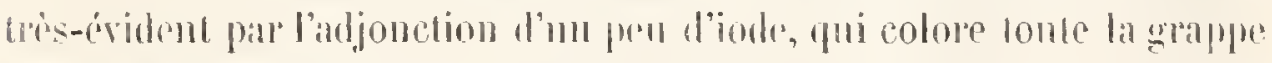
llin brom intense.

Il anve sourent que la lacho germinalive disparait dans le has de

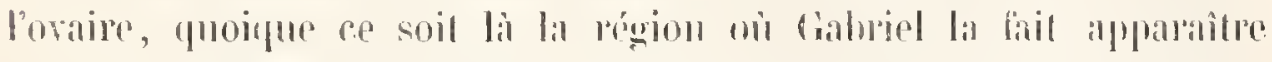

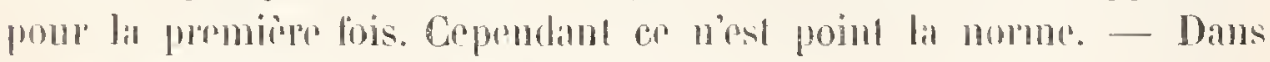

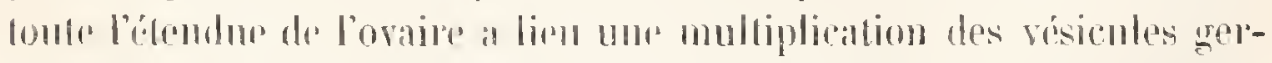

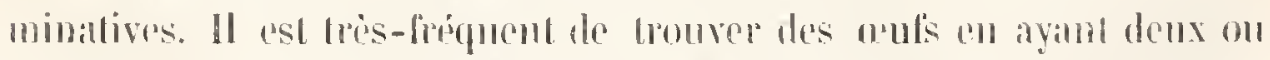

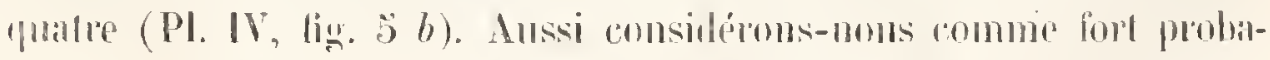

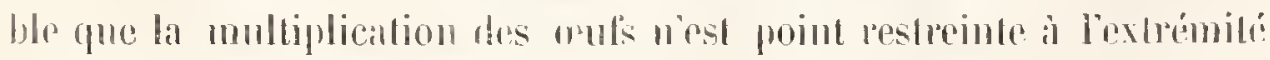

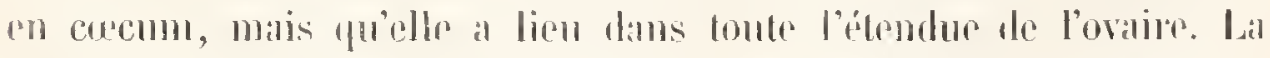

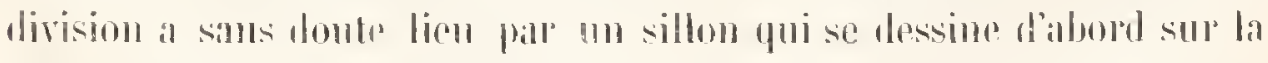

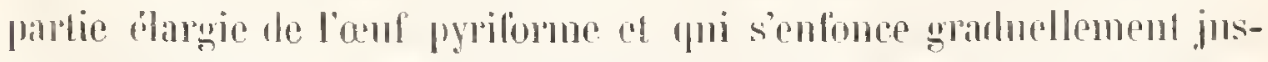
qu'au prodoncule. - Cai of li se trouvent anssi entre les auts des ve- 
sicules germinthive libres, nom entumbers de vitellus (Pl. IV, ligg. 2 al).

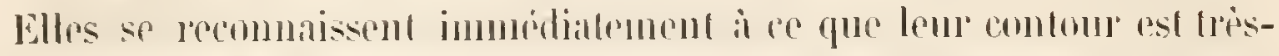

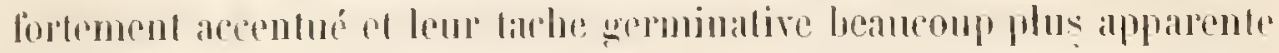

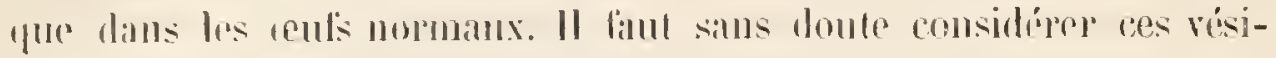
coles commme des ovules altophliris.

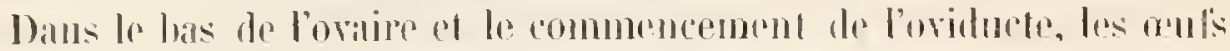
qui viennent de se ditacher du rachis lamifir conservent encore lent lome de proire. mais peu à peu ils śmrondissent a finissent par prendre none forme parfaitement sphérique (PI. V, fig̣. 6). Its ont alors un

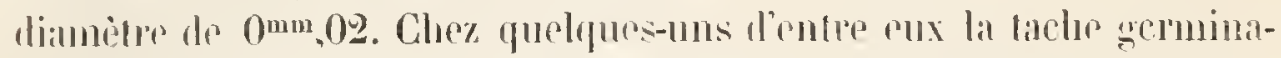
tim a dispart. Chez lo plus grand nombre elle existe encore. Elle a mème en général alors une ipparence vésiculeuse el renferme un pedit rorpuscule. On trome partois encope ici des ants munis de deux on de quatre résicules germinatives (Pl. IV, fig. 6, a), dre sorte quil nest pas impossible quime multiplication pall division ail lieu chez les ipule déjà arrivés à maturilé.

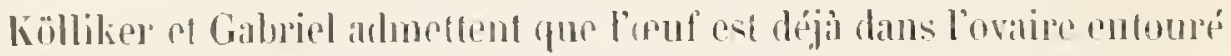
dome membrane vilelline. Cotfe monbrane résiste meme, dipris Galuiel, anx acides les plus concen!res. Cependint nous aimerions mioux ne pas nons prononcer ansi positivement it cel égard. Nons royons hien phuot tronver ici la meme disposition tue chez l'Ascaris suilla et

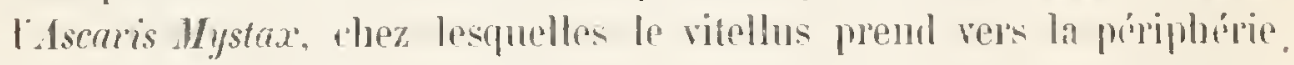
mo consistance un peu plus dromer. Corst, dams lons les cas, à bort que Kölliker el Gabriel admettent dewx membranes autom de rauf apris la lécondation, 11 thorion of mon memb!ane viteltine. Nais cest un sujel que nous reprendrons aillems.

la seconde catégorio pue nous aroms établie chrz les mématodes par rapport it la formation ales aduls comprend les especes chez lesquelies

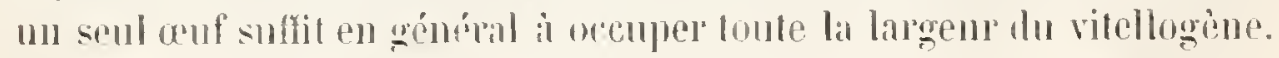
Le morle de formation des auls chez les nematodes appartenant it of

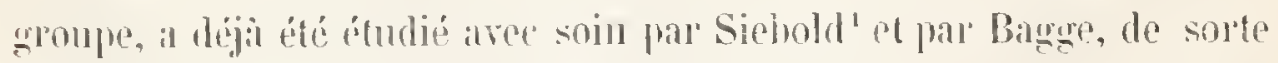
que noms ne nous en ormperons qur brièrment.

I Rurlach's Physiologie. Bd. 11, p. 209. 
Las especes que nous avons particulicrentent choisies pour to sujet de mos observaltious soml b. Strongylus auricularis, l'Ascuris nigrovenosa, l'Ascaris commutata el un mématode tromvé en assez gramde abomilance dans l'intestin do l'Hydrophilus picens, mimatode que Györy a lécril l'an lemiter (1800) soms le 110m d'Oxyuris spirolheca".

Cliez loms ees vers, le blistogène est oceupé parr des vésicules comme dims le groupe précédent, ansi que Sicbold et Bagge l’ond téji recomnu. Ces vésicules sont dr momran les vésicules germinalives arec lem tache te Wagner. Chez lo Strongylus auricularis te fond le la parlie areugle

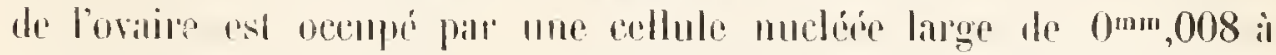

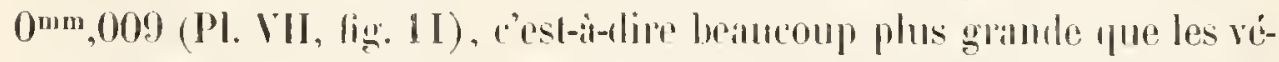

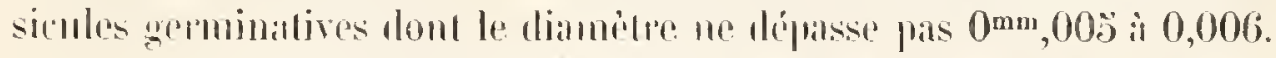

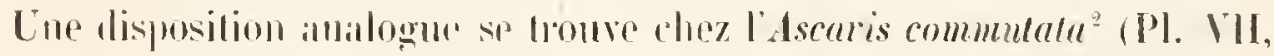

'Silzungsberiehte der h. h. Akatemie der Wissenselathen in Wien, Ba. XXI. Heft II Juli 1856, p. 327 .

${ }^{2}$ L'Asearide yue nous alrons désignée dans ee travail suls le nom d'A. commulata se troure en abundance à IBerlin dans l'intestin du crapaud commun (Bufo cinereus). Nous l'avous également trousée dans l'inestin de la grenouille ronsse. Elle ne coüncide exactement arec ancune les especes décrites par Diesing et Dujardin. Elte est de la ralle de l'A. acuminata. mais la yueue du mâle est mumie d'épines larges el conrtes. Des épines analogues son disséminées sur toute la surface du corps. La queuc de la femelle est d'ordinaire sans ápines, mais le corps est le plus souvent muni d'élévations pointues semblables à celles du mâle, quoique moins saillantes. Parfois aussi ces élévations manquent. Le earactere le plus saillant consiste dans lexistence d'une aile membrineuse de change coté du eorps. Cette aile, assez large dans la partio pustérieure du eorps, va en diminuant gradueltement jusque vers la tête, oì elle disparaît complétement. Diesing ne mentionne cetie aile ni chezl'A. acuminata ni che\% l'A. rommuata. Dnjardin signale bien deux membranes latérales chezson Ifeterakis brexicandato, mais la ligure yu'il donte ne concorde pas du tout avee notre ver, et de plus les deux membranes ne doivent exister que chez les mâles, tandis yue clez notre Ascaride elles existent dans les deux sexes. Nous arons choisi le nom d'A. rommulata parce que Diesing attribur au mîle de cente espere une queue munic de deux rangées de papilles. Cependant la descriplion de ces papilles ne concorde pas non plus précisément avec ce gue nous royons chez notre $A$ scaride. Aussi n'est-ce qu'arec doute que nous appliquons à cette dernière te nom d'Ascuris commutate. Diesingr a rencontré son asc. commulata dans l’intestin du crapaud rend (Bufo) ciridisł. Le mâle de notre espèce atteint environ le tiers de la longueur du corps de la femellr. Les spicules sont longs de $0^{\mathrm{mm}}, \mathbf{2}$. L'onverture vaginale est située à peu près exactement au milieu de la longueur du corps. 
lig. 7). Nous ne pourons pas plus ici que dans lis las précédents dire

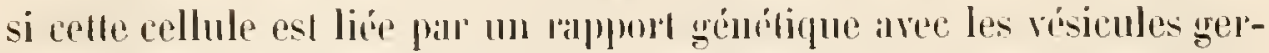
minafives, si elle les engender, en un mot. En fout cas, elle parait appantenio lien décilément à la paroi do lube générateur el ne doit

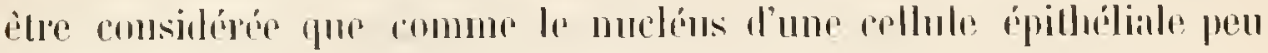
distince.

Pirlois, surtout che\% liscaris nigrovenosa, il linst pas pussible de dislingne les taches germinalives dams le blaslogene, mais on peut

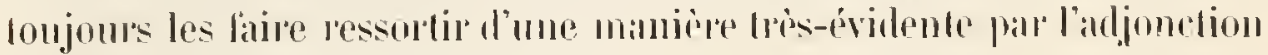
d'un peu d'acidle acélique éleudu.

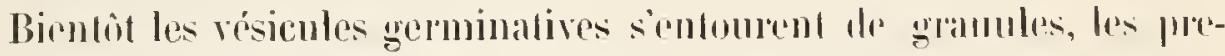
miers granules vitellins. Malis ici, pas plus qua dins le grompe précé-

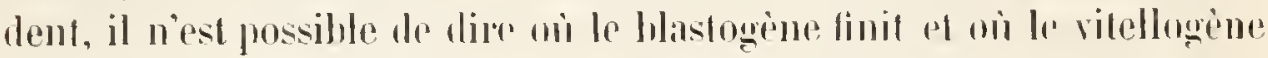

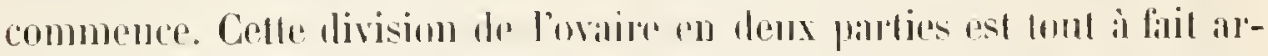
hitraile. Lat sulsstame incolore qui rntome les vesicules germinatives dans le blaslogène est dejà le premier rubliment du vilellus, de a de

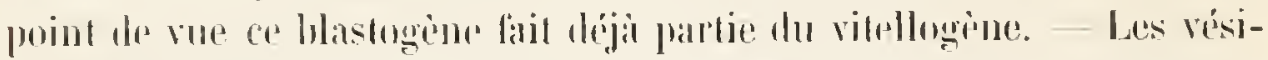

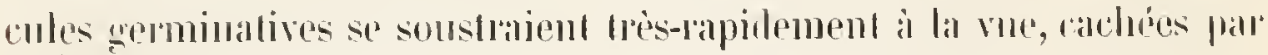

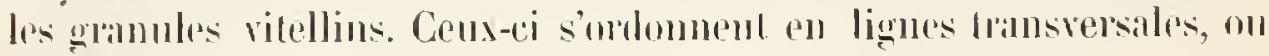
plutot en disques transiersintx forl minces dans l'ovaire (Pl. VII, fig. 12 et 15, du Strongylus anricularis). Ces disques si minces sont les jounes ants. Dabord indistincks, ils se dessinent par degrés d'ume manière phus ivilente. Dans la région on ces disques commencent it itue fort

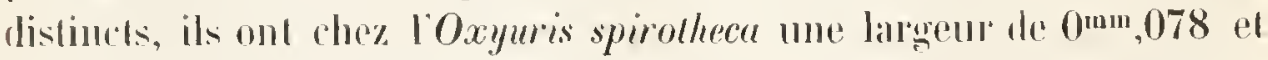

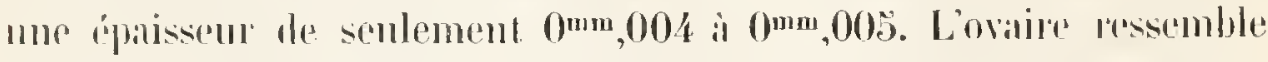
alors à une pila ì colonme, telle que colles qui furent construiles daus lorigine par Volta. Le plus somvent les éliments de la pile, c'est-

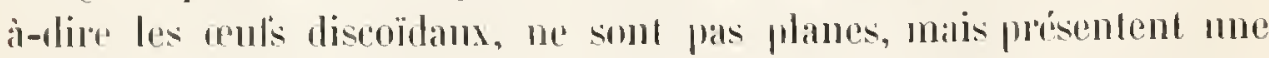

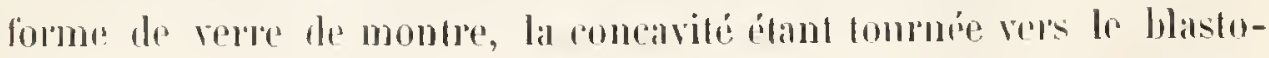
gene of la convexití vers lontérus. A mesure quon gagno les rógions inferieures de l'ovaire, on roit les disques choitre en ápaisseur. Parlois ils sont si pressés les mns rontre les autres quil est fort difficile de reconnaitre les limites de chatue aul. Mais il est mn moyen fort 
simple de démontrer que les aufs sont à ce moment parfaitement distimels les mos des antres. Il suffit en elfol de plonger lovaire dans une dissolution de sel mariu un pen roncentréc. Clıaque oul'se contracie alors pour son propre compte of so sépape de ses voisins, tout all conservint sil lorme générale. En mòme temps la coulenr sombre de licent śrelaircit, of il en risulte que la vésicule gepminalive, quon ne pouvaif réussir i dislinguer anparavant, se laisse subitement voir de la manioure la plus évidente. Les figures 9 ef 10 de la planche VII representent fo mome fragmont do lovaire l'mor Ascoris commutata

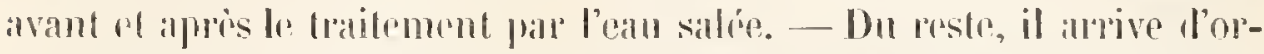
dinaire que la résionle gemmalive se montre d'plle-mone dams le bas dı vitellogène sams l'emploi d'ancun réartil. - Las disques mérilent ì w monent i juine rnere re nom. Ils se somt considerablement ćpaissis an centre, tomt rat restant relativement mịnees à lenr pépiphérie. La

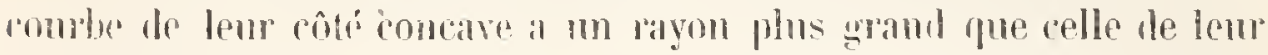

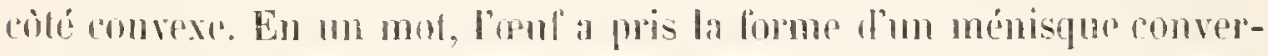
gent (PI. VII, lige. 14, Strongylus auricularis).

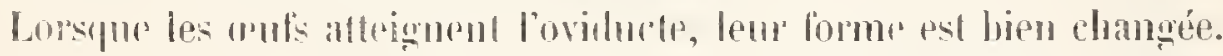
Dans les tregions supépicures dn vitollogène ils rtaient 20 on 50 fois anssi larges pue longs; maintenant, an contrabre, ils sont phos longes

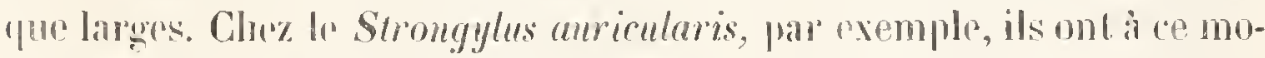
ment li) lopme do rylindres hantis de $0^{\mathrm{mm}, 09}$ of liarges de $0^{\mathrm{mm}}, 05$ (Pl.

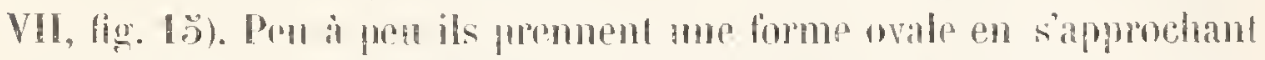

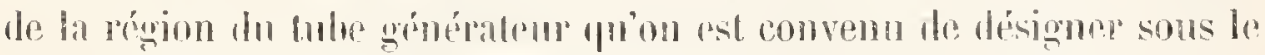

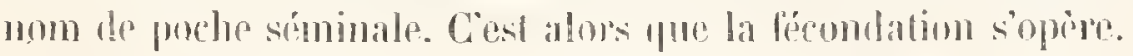

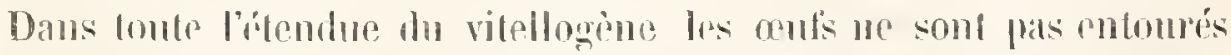
d'me membiane proprement dite, lien que la surface pxterne du vitellus pusse aroir une romsistance plus dense que le reste. Ce nest quapres que la fícondation a ru lien du'on voit apparaitre une memlowne incontestable. Bagege panail copendant avoir admis me membrane autour de louf des fo commencement du vitcllogène. II dit en rlfet: Postquem vesicula germinativa evunuil et granula ad centrum magis sinl conferla, vilellus preter eam que nuiversum onm circumdans adhuc 
conspiciebatur. propria inchudim Iunicu, quam cerisimile est jum antea subfuisse, sed intermo lateri membranu commuis ovi ita adhorentem ut oculis distingui ommimo non posset. Bangge anmait sans doute en the la

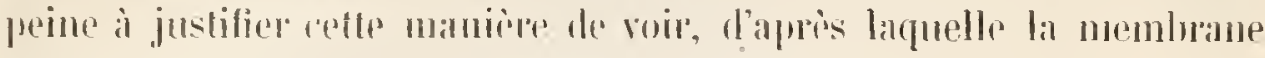
vitelline apparaitrait plus land fure lo ehorion.

Soit ehez l'Ascaris commuluta soit cluz. l'Ascuris migrovenosa, l'm ronrontre fiéquemment phusieurs rents dans une mème section transversale de lovaire, sourent deux, parlois trois, et mème quatre. Cependant il ne peut y aroir de doute sur la cácígorie à laquelle appartiement ces especes, panre quon tronve tonjours une partie plus ou moins longue du vitellogènc qui n'adnet quin cenf dans sa larweur. La partie inférieure đlu vitellogène s'élarọil considérablenent, et il en résulte que l'empilement rógulier des rufs so dérange ef que plusients ant's se plarent à colé les mo les autres an mème niveau. L'adjonetion d'eau salée qui fail contracter les unds, de manière à ce quim espace libre se lorme entre eux, permet facilement de recomaître que les apul's ne sont pas adlérents les mo aux autres dans ce cas, ef quil n'y a pas trace de rachis. - Nous avons remarqué que les Ascaris commulala de petitr taille rout jamais quome seule rangée d’rufs longitudinale dans toute l'étendue de l'ovaire. Les individus plus gros, chez lesquels les organes sexuels ont pu acquérir une plus grande langeur, montrent en général plusicurs oufs dans une seetion transversale des parties inlérienres du ritellogène.

Du reste, les deux catégoriesque nous arons distinģuées daus le mode. de lormation les wuls, ne sont pas anssi essentiellement dillërentes l'une de l'anlre qu’elles en ont l’air. Bien qu'on ne troure pas de rachis dans la secomle catégorie, eel orwane y existe rependant virtuellenent. l'agglonération même des ovules encore peu différenciés dans la partie supérieure du vitchlogène s'expliqgue en quelque sorte par l'existence liun ratehis diffus. 


\section{DE LA FORMATION DES ZOOSPERMES OU CORPUSCULES SEMINAUX.}

Nous refrourons, à propos de la formation dles zoospermes, un déhal parlaitement analogne à celui que nons avons lajji vol se téronler au sujel de la lormation des aufs. Les mus veulent que les corpuscules séminaux soient dis fent premirre olignine enveloppés l'une membrane; les intres, in rontaire, prétendent quils sont pendant me grande partie de lenr développement dépourvus do lonte memlurane cxlérienre; les uns trouvent l'extrémité areugle du leslicule (l'aualogue du blastogrinc) remplie le víribales cellules mucléées el mucléolées, les autres

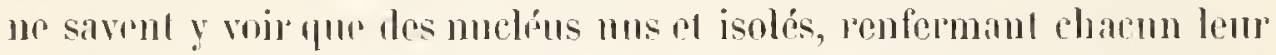
mucléole. La première opinion a trouré des défensems dans Reichert

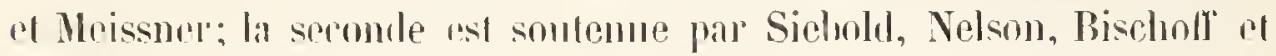
Thompson.

Le center de la disomssion est de nomvean ocenpe ici par l'Ascuris Mystux. Mallhemrusement, d'un wòté, les chats ne sont pas tris-abondants i Berlin, el d’un autre coite, chez la plupart dres nématodes, les miles sont relativement rares. Il est rísulté de lì que nous n’avons pas en l'occasion áfudier l'Ascaris Mystax male, les quolques chats que nous avous ens à notre disposition śétant tromvés ne renformer que des femelles. Mais ce n’est là que demi mal. En effir, nous avons en entre les mains plusieme indivilus miles appartenant à l'Asceris suilla du pore. Les drux Asearides enl yuestion sont riddemment assez proches parentes pour qu'il soil licile détendre il l'une les observalions

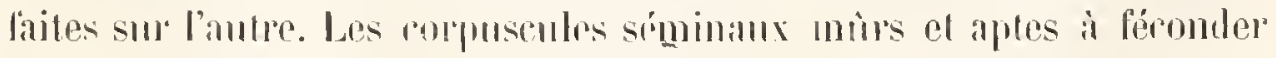
sont si semblibles antre mx chez des demx espreces qu’il n'est pas possible de les distingmer (V. PI. V, fign. 10 les zoospermes mùrs le l'A. suilla el tig. II cenx de l'A. Mystax). Anssi n'est-il pas dontenx que ces corpuscules ne se développent d'une maniere parfailement illentique dans l'une el dams l'antre espèce. 
Il y a dans le fail mo homologgie complite ontre lo développement

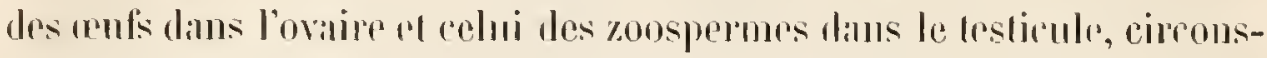

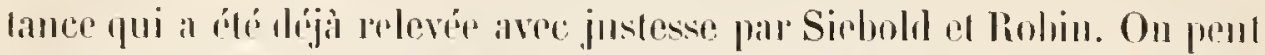

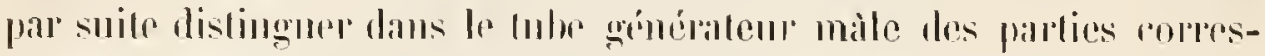
pomlant exalement an blaslogène, an vilollogine, à l'ovidurle, ì l'ulé-

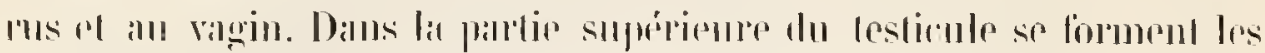

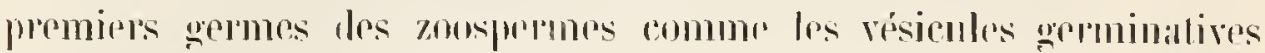

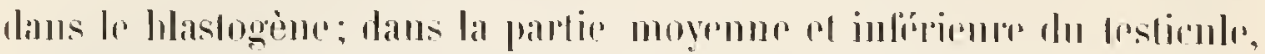

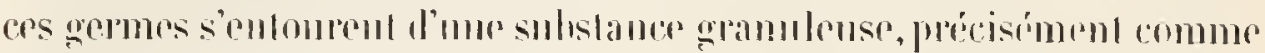

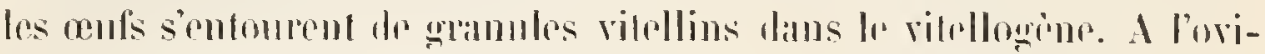

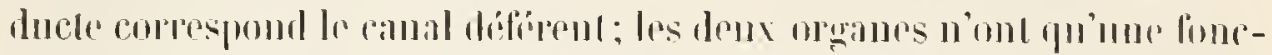

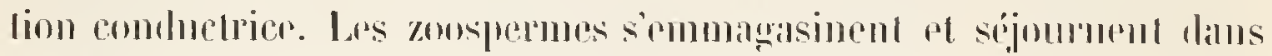

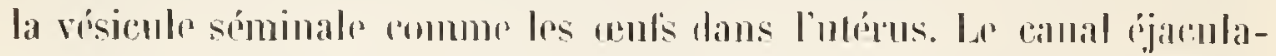

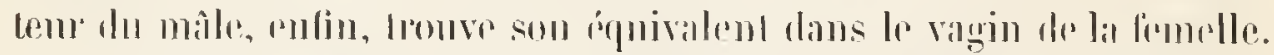

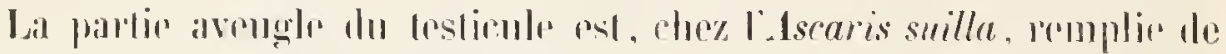

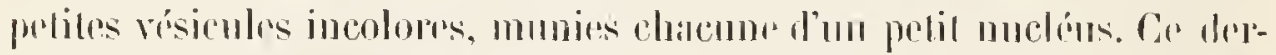

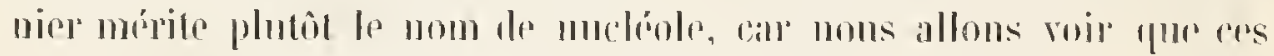

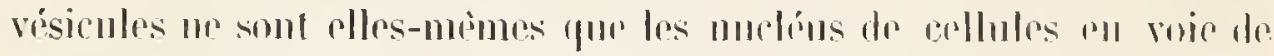

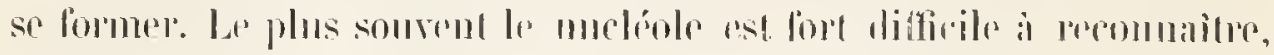
of lou rooit n'avoir devant soi que de simples résicules, romme des

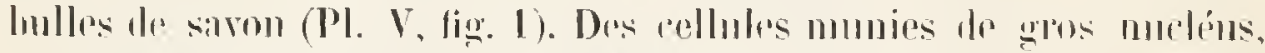

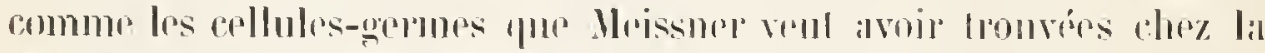
Mermis albicans of l'Ascuris Myslax:', nexistanl tris-centainement pas dams te teslicule de l'Asemis suilla. Anssi ne doutoms-mons pas que

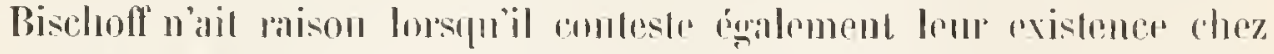

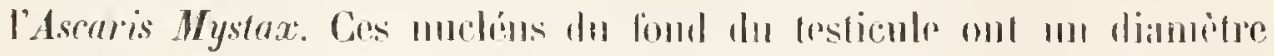

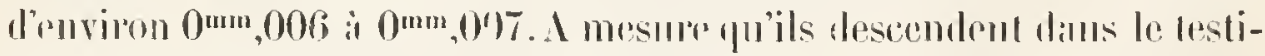

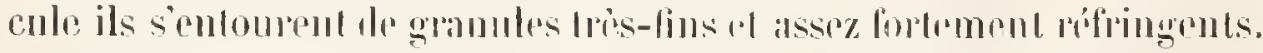

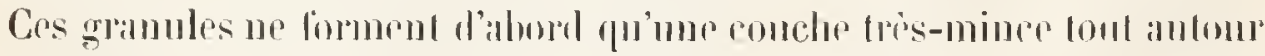
du mucléns (Pl. V, fig. 2). La (purslion de savoir si res gholules ainsi

\footnotetext{
1 Beitrïge zur Analonie und Physiologie von Mermis albicans; loc. cil., 1. 239 et suiv. Beolachlungen über das Eindringen dar Samenelemente in den Dotter; loc. cit., p. 209.
} 
formes sont limiles par une membrane externe on non, est de nouveau ici prufatement oiscuse. Meissmer lablirme, Bischolï le nie. Pour ce

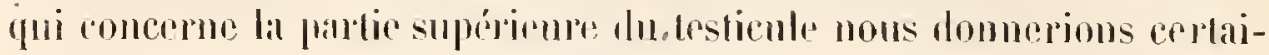

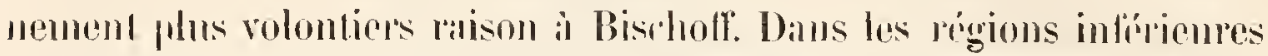

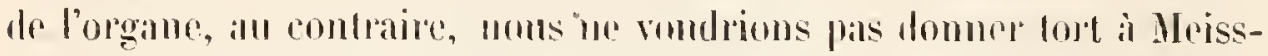
nur. La sulstance qui rofome les muchéns se comprose précisćment

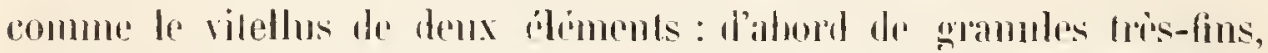
analogmes anx gramules vitellins, phis d'me substamer tramsparente el

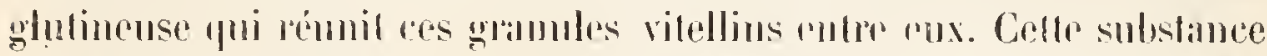

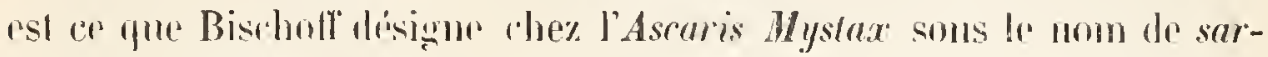

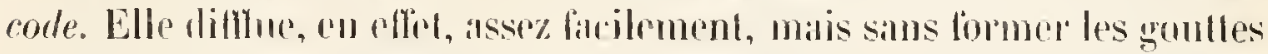

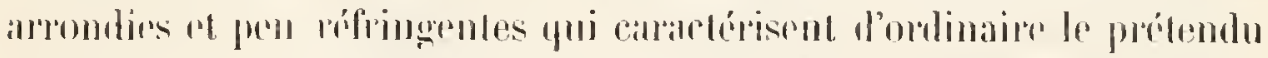

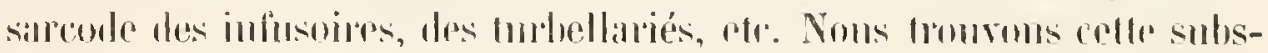
lance plus dense il la pépiptréric quan centre, at si nous ne ponvons

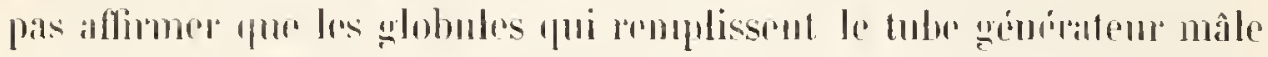

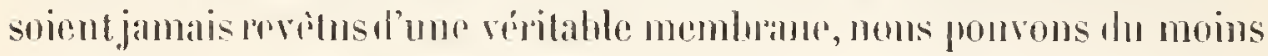

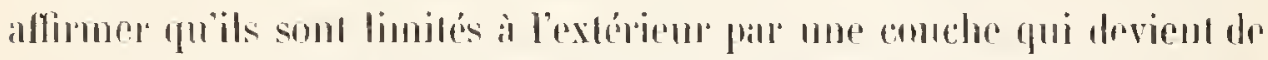

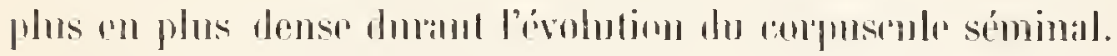

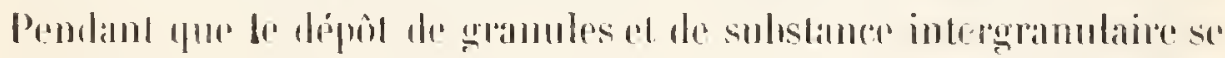

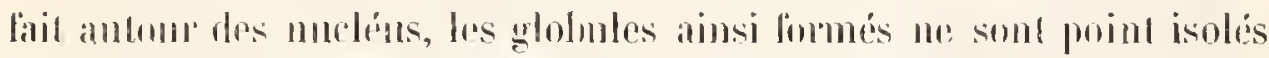

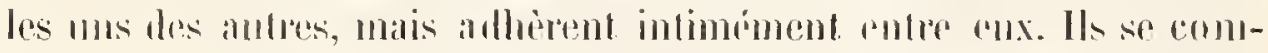

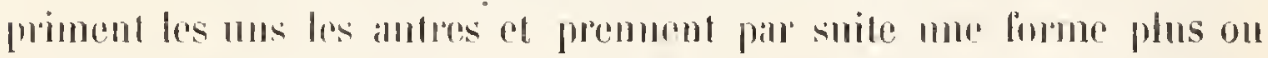

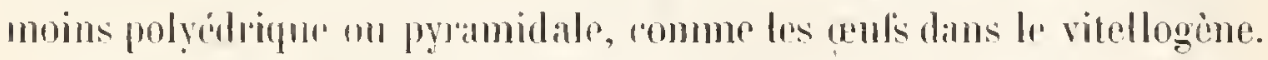

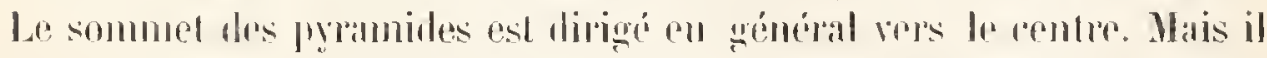

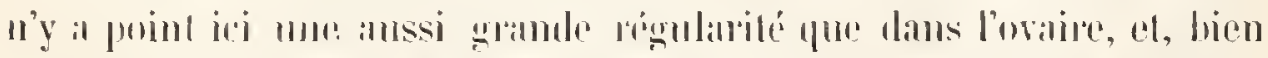

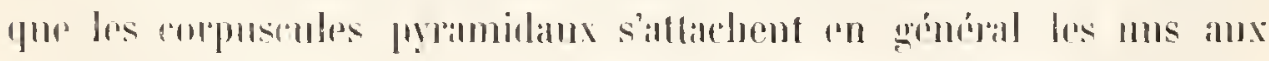

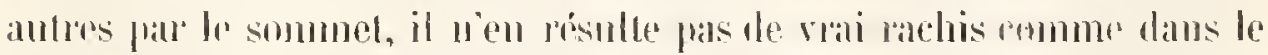

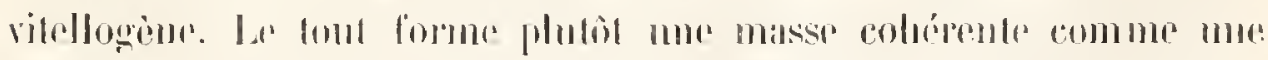
mìn on 110 framboise, masse dont les éléments so séparent eependamt asseg farilement les uns des antres (Pl. V, lig. 2). Ceffr formation

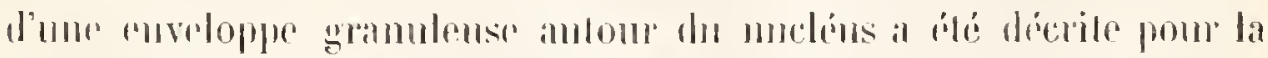

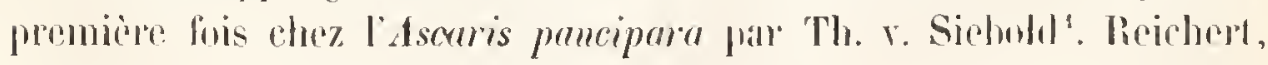

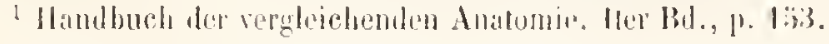


fall snite de ses observaltions sur lo Strongylus anricnlaris of l'Ase. acuminata, a cru devoir contester l'exactitule de la description dommer par

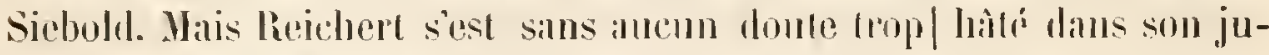
gement. L'Ascaris pancipara renfirme, an dire de Siebold, des éléments

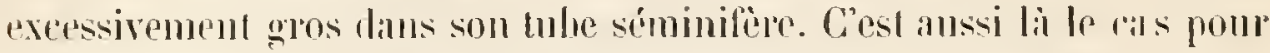
l'Ascaris suilla, ou ces díments atteignent dans le milien du testicule

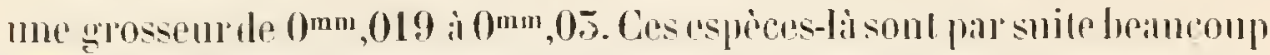
plus fiurorables i l'átude du sujed qui nous oceupe que te Stromgylus anricularis of l'Asc. acmumata. Nons croyons pouvoir alfirmer ave une

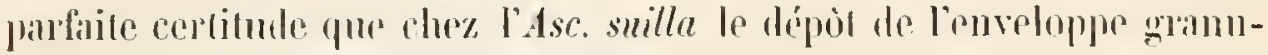

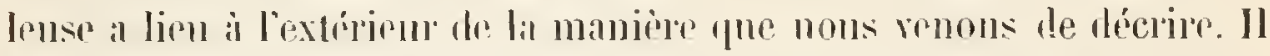
déconle do li mime yur les ohsepralions amalogues de Siebolel chez

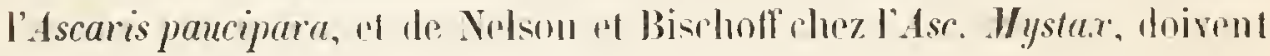
acquérir une grande vaisemblanor.

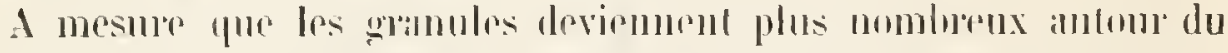
unclés, celui-ci devient plus diflirile i recomaitre. Une teinte phos

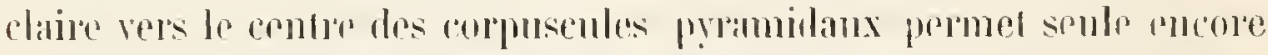
de constater sil présence. Cependant, l'adjonction l'me goulte d'aciele

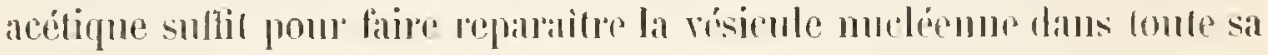
splendem (PI. V, fig. 5). Les gramules perdent considerablement de

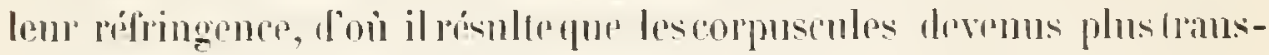

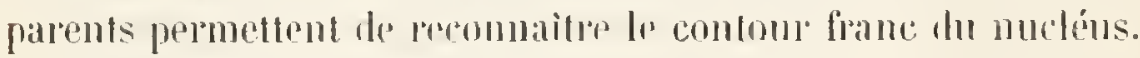

Plus bis les corpusenles pramidams se séparent les mos des antes, l'adlérence mutuclle asl délruite; an mime tomps la forme de ces cor-

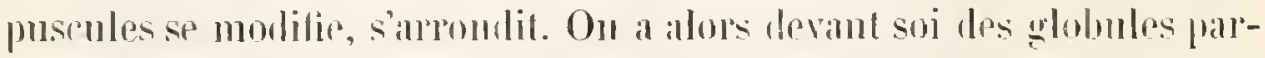
faitement sphépiques (Pl. V, fig. f). Ils sont plus pefits fun les pryalmides de maguèe, parrer que l'arrondiscement du roppusenle a été accompagne dime espero de rontration, de rondensalion. Anssi n'y a-t-il plus possibilití de reconmitite trace du nucléns perdu dans la masse gramuleuse. On pent cependant dímontrer encore prombant in certain temps l'existence de re nucléns avec le secoms de l'alcide acelique (PI. V, fig̨. ̈) . Il se dessine alors vaguement comme ume tache fâle, sans contonl's définis. Bientôt cependant il disparaît compléte- 


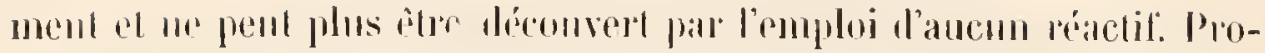
baldement que la résiculd bilate ou se flissont, al que son contenu se mébluga aree la sulstance mêner du corpuscule. Cest à ce moment

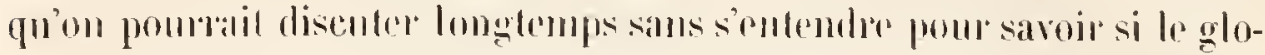

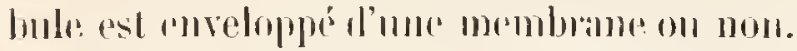

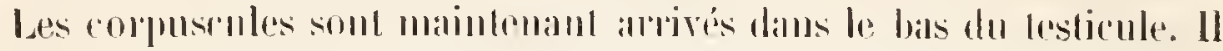

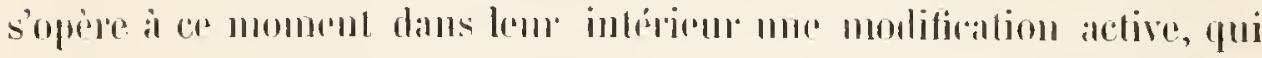

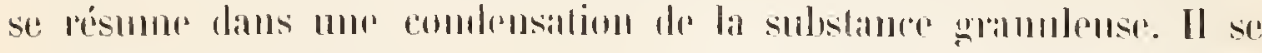

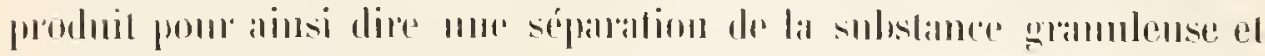

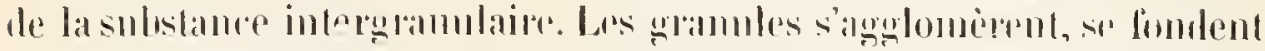

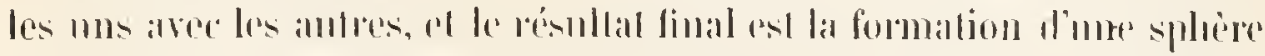

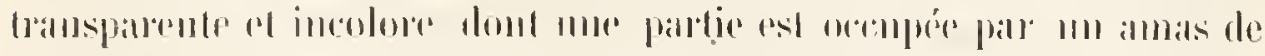

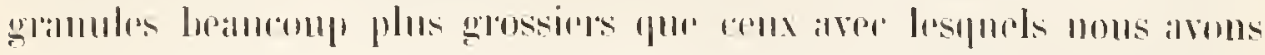

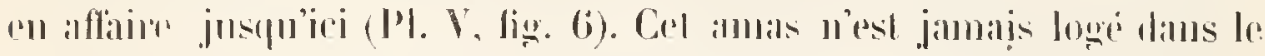

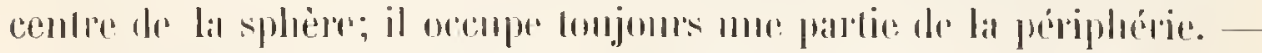

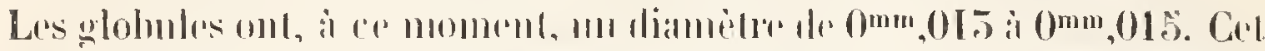

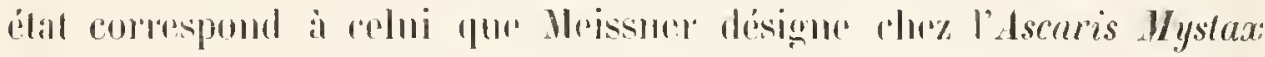

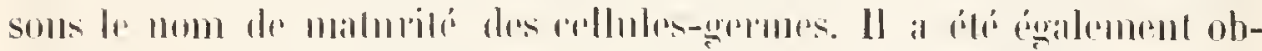

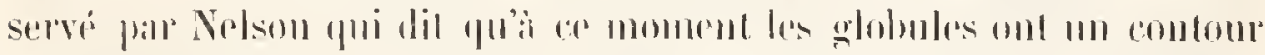

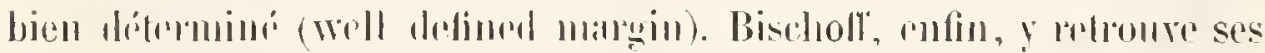

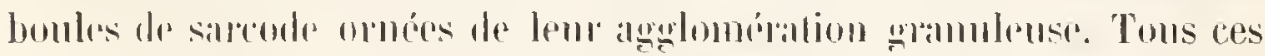

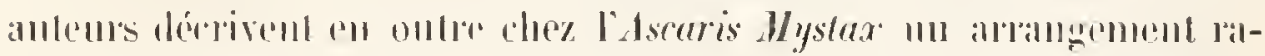

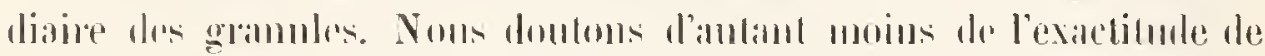

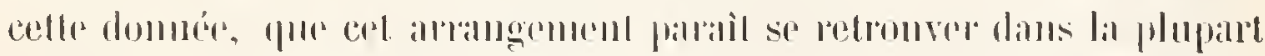
des mómalondes. Cependant il ne nows pas ré possible de constater d'une

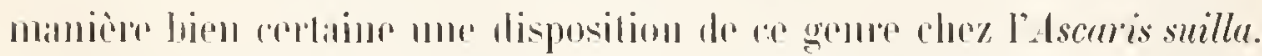

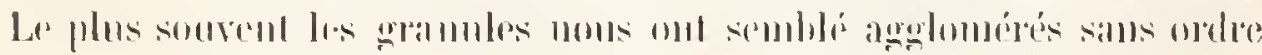
bien recommaissilule

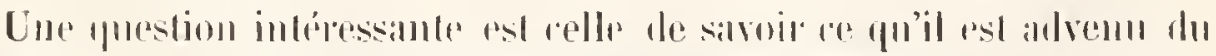

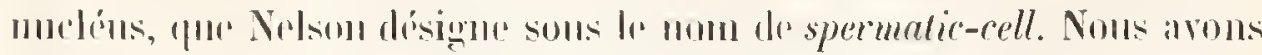

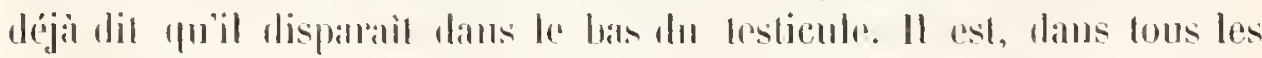
cas, cerlan quil n'an exisle plus later an montent où noms sommes. Noms pensoms dome que Nolson so trompe lorsquil adnet la persistance 
de ses spermatic-cells. A son avis, la formalion de l'enveloppe gyantuleuse lies que lemponaion; ollo disparait plus lard lomsque le corpuscule est arrivo dans les organes génitanx femelles, et met ainsi on liberté lat pretite vésieule, si lomgtemps prisomniere. Cest mo point sur lequel

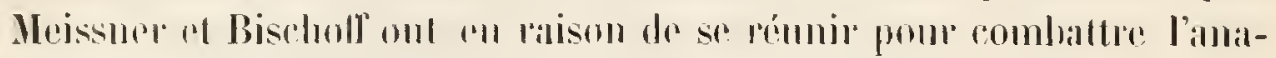
lomiste anghlis.

Les globules, dims l'élat mì noms venons de les dérire, sont suscep-

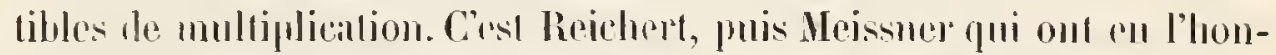

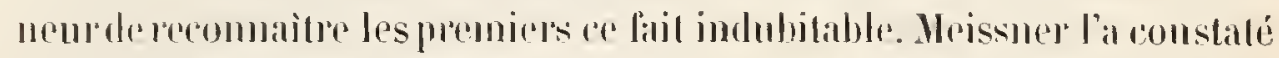
soil chez la Mermis albicoms, soil chez l'Ascaris Mystax, mais dans les

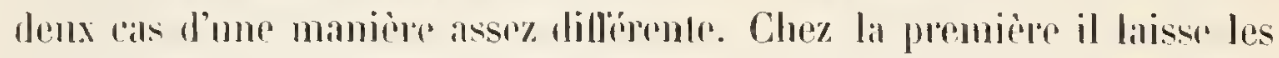
"uucléus " (évidemment l'analogne dre l'amas des gavanules chez les Ascarjes) se multiplier dans la cellule-germe. Il s'en lormo d'abord

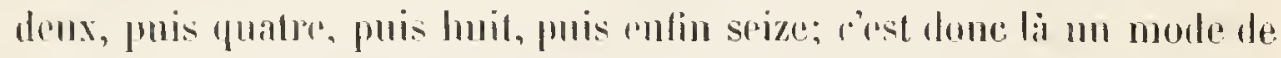

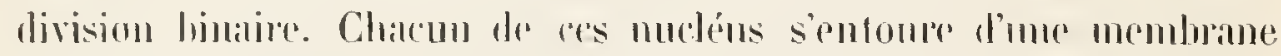

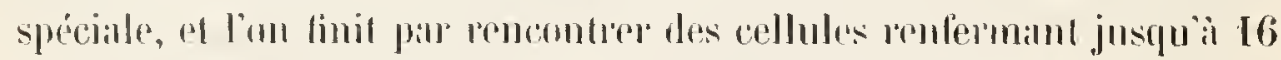

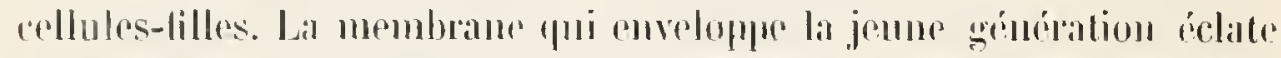

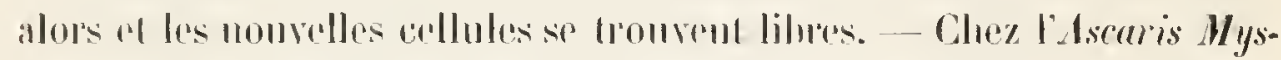

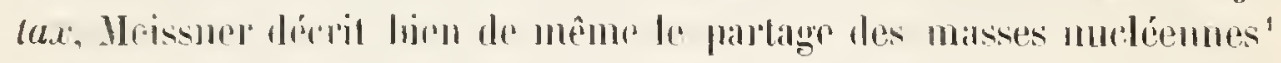
(amas gramulenx) en deux parties, el davintage, jusqu’i lunil parties;

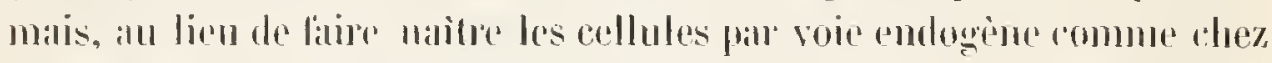

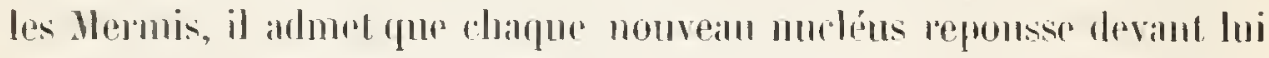

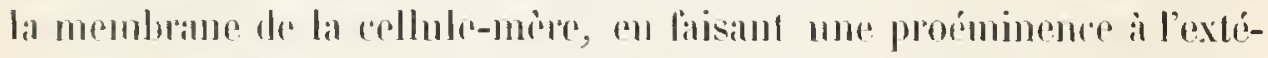

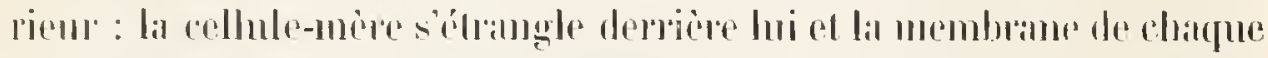

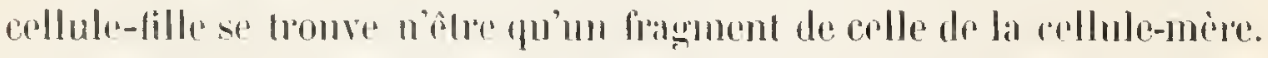

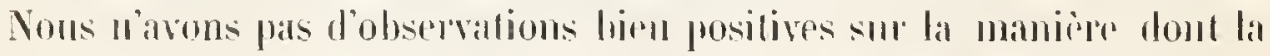
lormation des rellules-filles a lien chez l'Ascaris suilla. Mais d'apres ce

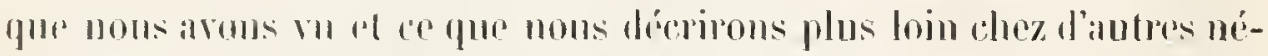
matodes, nous pensoms que te modr de multiplication des glohules

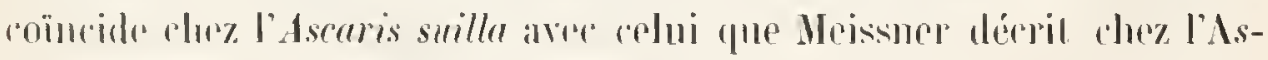

\footnotetext{
1 Sous ne woyus du reste pas trop pumpuni Meissner assimile ces amas granuleus a des uucléus.
} 
caris Mystax, arec celte modification que la soi-disant membrame u'est pent-itre pas complítrment différencire du globule comme membrane.

Les difléreuts observateurs s'ilcordent à dire que le développement

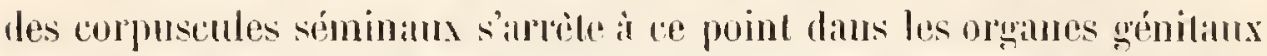
miles, quithe a proursuive sil marche me lois que as corpuscules sont

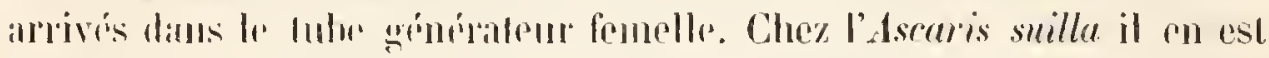
antrement. Les ghobules gur nous venons de decrire at qui sont l'ho-

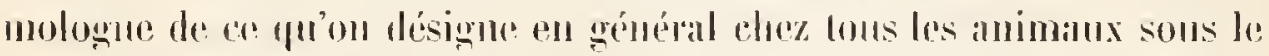

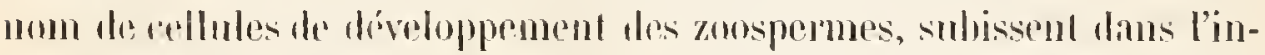

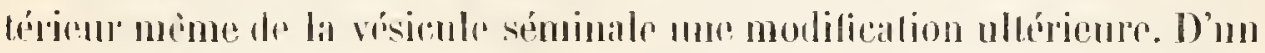
point queleonyue do la misse gramuleuse, on voil sélever mor légère

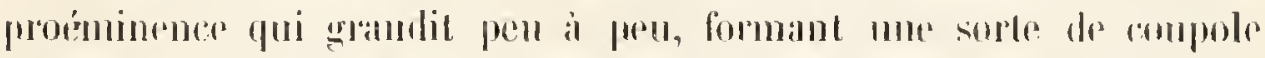

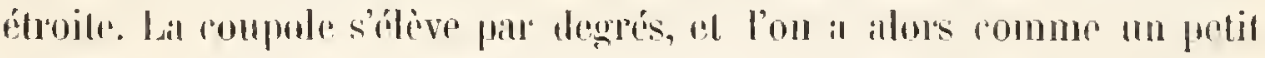

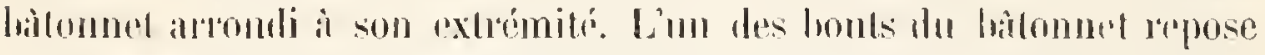

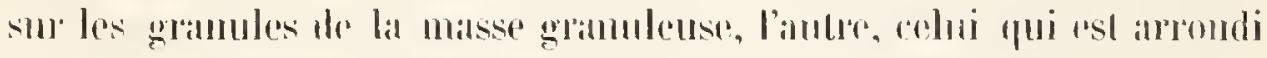

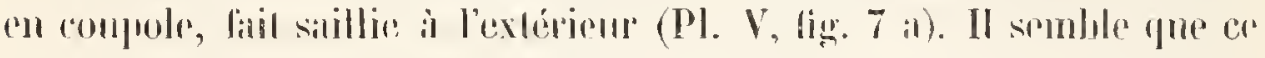
doje bure dei un moment propice pour décider si lis rellule de dévelop-

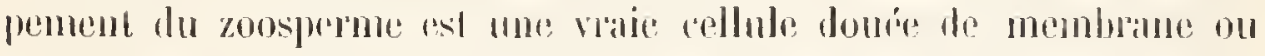
simplentent me boule de sarcode. Sil existat me membrame, on de-

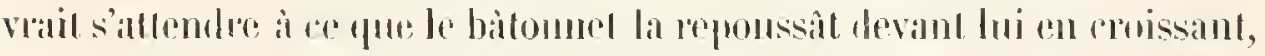

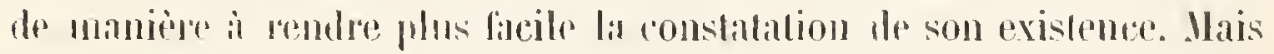

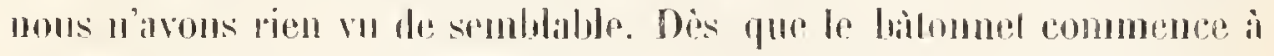
se développer, la partire trinsparrente a incolore de la cellule de déve-

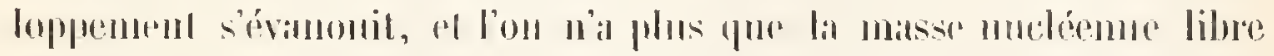

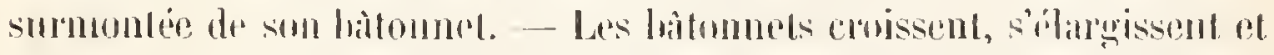

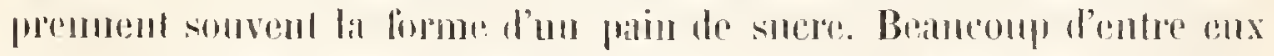

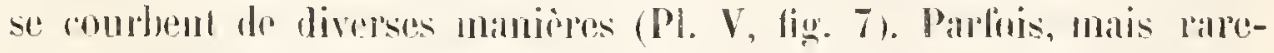

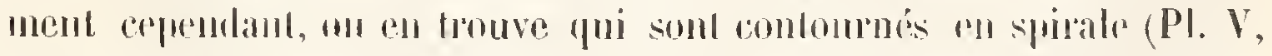

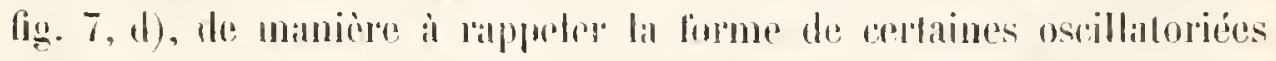

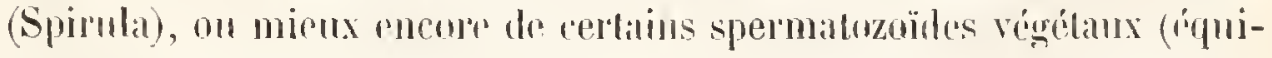

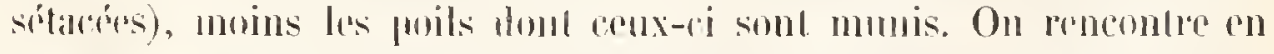

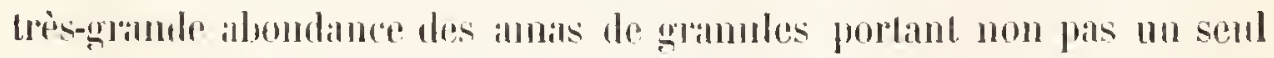

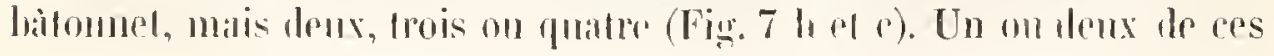


derniress sont somrent pacesirement maighes, comme alrophiés. Nous

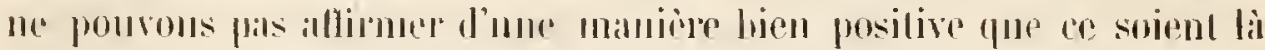

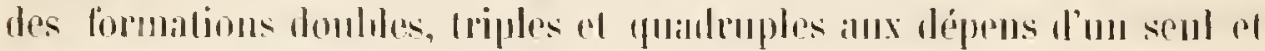

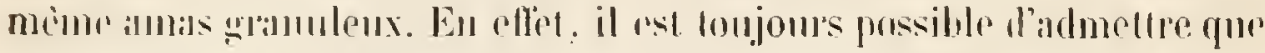

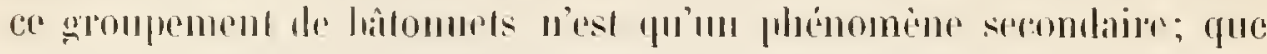

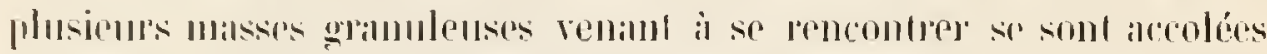
les mes anx intres el firment des Jors comme un loul connpiat. Cepen-

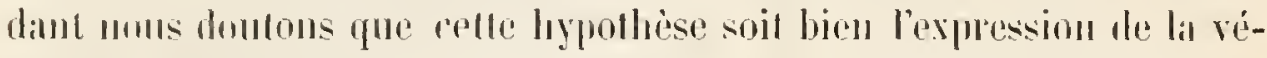

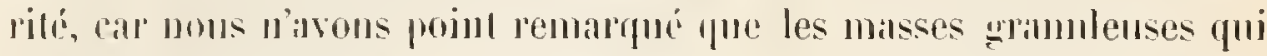
portaient deux bitommets ou davantage liussoul plus grosses que celles qui n’en portalent qu'un. - Entin. on trouve dans la vésirule síminale des rorpusenes de forme direse, en général plus ou moins baculitor-

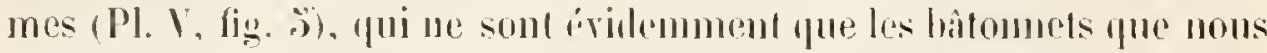
renoms de décride, mais doul la base es détarrassée de son amas de granules. Quelques-mus sont bizarement contommés (Fig. Z, b), mais la phupart rippellent par leur conformation lat lorme d’un doigh lum-

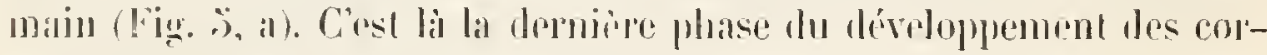
puscules siminanx que nous aỵons obscrée daus l'intérieur des orgáu's miles. De ces corpuscules baruliformes aux zospermes rels que nous las retroureron pho loin dans loviducte des lemelles, il n'y i

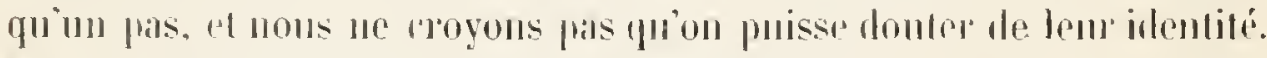
Mais cest mor qurstion que nous reprendrons.

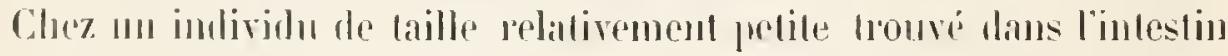
du frote a pur nous considéroms comme un jeme individu mâle de

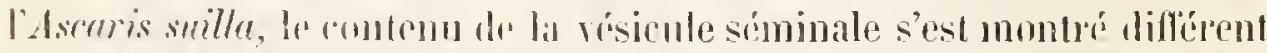

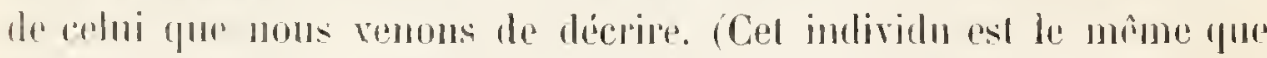

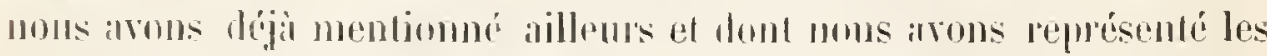

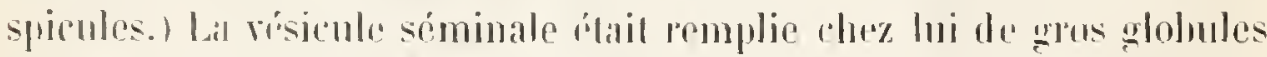

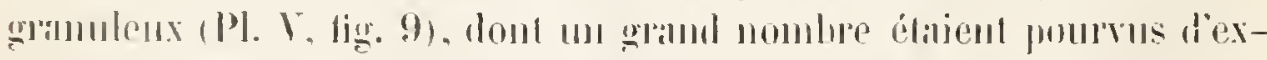
parsion incolores, non gramulanses, assez irrégulières. Ces glolumles

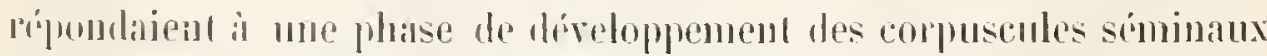
que nons arons dijji décrile el que nous arons représentée dans la fi-

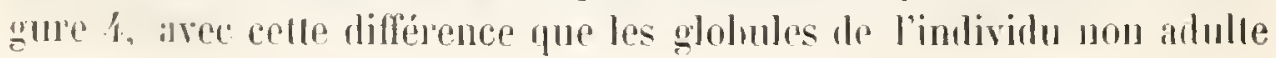


étaient munies des expansions prócitées. Il serait peut-c̈tre possible que ces expansions no fussent pas une produclion normale, mais qu'clles eussent été engendrées par l'action du liquide dans lequel les globules furent olssepres. Bischoff" décril in eflet des formations foul analognes chez les corpuseules síminamx de l'Ascaris Mysax sous l'influence de l'eau pure, el il les consilère comme dus émissions de sarcorle. Ce serait lì, suivant lui, le premier starle de la dissolution du corpuscule. Ceprendant il est juste de dire que Mleissner a déclaré n’avour rien pu voir de semblable rhez l'Ascuris Myslax, et nous-mèmes nous n’avons jamais vu se formur de simblables rimissions de sillcode chez les corpusenles síminaux de l'Ascaris suilla, re qui s'explique pent-ifre par Ir fail que nous avoms mesque tonjours observé ces corpuscules dans mor dissolution de sel marin on de sncre de camme. Les glohules de notre jemme Ascaridr présentaicnt, an contraire, leurs expansions mime dans une dissolution salver, sims quill fiil cependimt possible de constaler des phénomines d'émission ateliro of de rátaction, semblables ì cenx que Schmoirlor a constatés chez les eorpuseules séminam de divers nématodes. Aussi croyons-nous devoir immoflle qur los globules éainnt normalement pourvus des expansions an question. Sous ne prótendons point par la susperter l'exaldilude des observations de Bischotr, call il est facile de voil chry certains nomatodes (Ascaris rommulata, Cucullamus elegaus, ele.) des phónomenes parfailement identiques ì coux

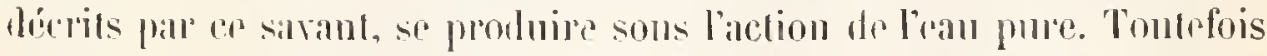
nous considrírons les globules an question commo des rorpuscules sémiman an milien de leur développement, rorpusenles qui u'araient pas

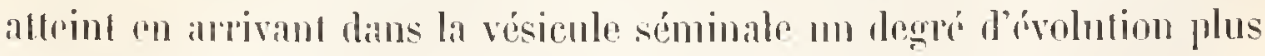
araneé qur celui quion reneontre d'ordinaire dans le has du testicule.

Bischoll rapporte avoir trouve pour ha premiè fois, en mars 18:34, chez plusieurs miles de l'Ascaris Mystax d'mutres corpuscules que les globules ordinaires. Ils riaient, d'apris sa description, lortement riffingents, jiunitres, luisauts, longs de 1/150 ì $1 / 255^{3 m m}$. Ces corpuscules étaient cylindripues el existaient aussi dans les organes génilaux des femelles. Bischofl les iefromva dr nourean en Arril el mo .tullet, mais 
sans oser décider si ces corpuscules sont des zoospermes, des psemdoplasmes ou des psorospermies. It paraìt cependant incliner plutôt ì y roir les rais zoospermes. A notre avis, il n'est pas impossible que res corpuscules soient précisément les bàtommets que nous arons décrits che\% l'Ascaris snilla, et dams ce cas Bischoff anrait parlaitement laison dans son hypothèse. Cependant ces zoospermes ne serairnt point mùrs el aptes i féconder, mais seraient seulement me forme de transition entre les globules ordinaires du tube génératentr mâle ot la forme définitive des zoospermes donl Bischoff a fait ses comules épithéliaux.

Nons avous étudié comme terme de romparaison la formation des zoospermes chez d'autres nématodes que l'Ascaris suilla. Les corpuscules séminaux de beameoup de ees ver's sont te trop petite laille pour permettre de seruter avee avantage leur morle do dévoloppement. T'el est le cas, par exemple, four ceux du Cucullamis elegans. D'antres, sans alteindre des rlimensions bien ronsidérables, oftrent cependant déjà des contitions phus propices. Nous arons choisi pour sujet de notre description les corpuscules séminaux du Strongyhs amricularis, te l'Asraris commutata el de l'Ascaris mucronata.

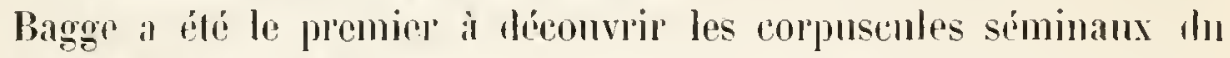
Strongylus auricularis, mais e'est à Reichert qu'il élait réservé d'étulier pour la première fois leur développement. Comme on le verra, notre deseription s'écarte considérablement de celle lonnéc par ce dernier savant. Les causes qui ont amenć ces lifférences dans les résultats sont lle diverse nature. En particulier, Reichert s'est peut-îte parfois laissé trop facilement dominer par tes irlées throrigues sur la formation de la cellule. Puis ce savant était préoccupé par la pensée do l'existence d'une homologie morphologiqur entre les corpuseules séminatx les nématodes et les zoospermes filiformes de la plupart des antres animaux. Or, nous ne croyons point ì la réalité de entle homologie morplrologique, ou du moins nous ne pensons jas qu'on puisse la retrouver jusque dams les plus petits détails, comme Reiehert l'a voulu. Il existe, nous le croyons, une homologie physiologique parfaite entre les corpusenles séminaux des nematorles et les zoospermes filibormes, mais 
l'homologie morphologique doit être réhuite à une simple homologic génétique, qui ne repose pas mêne sur des bases bien solides. Il est possible, en effet, que les parties rssentielles du corpuscule séminal définilil soient dérivées du nucléus de la rellule de développement, re qui indiquerait une analoģie d'érolution avec les zoospermes filiformes, pnisque cenx-ci paraissent se former en général anx dépens du nucléus de la cellule de développement. Cependant il s'agirail encore de démonher jusqu'i quel point on pent domner, arec Meissner, le nom de

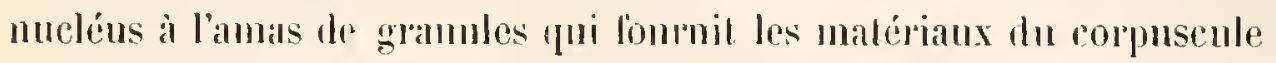
séminal cléfinitil. - Reichert distingue rhez los zoospermes du Stromgylus auricularis mo tète et me pucue, précisément rommo chez beancomp de zoospernes filiformes. Mais la romparaism do ces parties avec celles pui portent le mène nom chez les zoospermes filiformes est-clle bien justifiable? Nons ne le pensons pas. La quene, en eflet, est che\% le zoosperme fililormu l'organe molum; clle s'agile romme une anguille el produit prar la la nalation du corpuscule. Coes, en mu mol, la parlie aclive an point de vue de la locomotion. Chez les zoospermes du Strongylus auricularis it en est foul difléremmonl. Nous amons phos loin l'necasion d'ćbulior les mourements de ces corpuscules, et noms reprons que la partie que Reichert appelle la quene, bien loin d'être active dans la locomotion, rst trainéce en arrière d'me manire purement passive. La partie motrice dans te corpuscule est précisíment celle que Reichen appelle la tête. Nons savons d'aillemrs que la têlo on la quenc ne sonl point denx partics essenticlles des zoospermes; nous n’avons qu’ì rappelor les zoospermes simplenent filiformes, sans ancun renllement i l'extromite, de tant de mollusepues of d'articoles.

La partie avengle du lesticule, Jarge l'environ (0mu,02, est remplie, deez le Strongylus auricularis, de vésicules jonissint d'im diamètre de

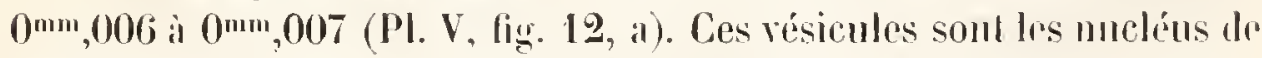
cellules en voie de se former. Chacume d’elles est mumic d'un mucléole. Le fond du coecum est l'ordinaire ocupé par unc vísicule plus large (0mm,01 environ en diamètre), qui paraîl apparlenir à la paroi du lube génépatenr (Fig. 22, b). On doit probablement la considérer romme” 
une cellule épithéliale'. L'espace libre yui subsiste entre les nucléus est rempli par me sulstance ì pen pris incolore légèrement gramulense. Cette substance devient toujours plus abondante ì mesure qu'on s'éloigne du fond du resticule, et Inrsqu'on dischire la paroi de l'organe on reconnail quelle forme mue enveloppe antom de chinge nucléus. Cette enveloppe us parait pas a ce moment etre limite par une membrane proprement dite. Elle lait bien plutiot l'impression diune conche de mucosité déposée sur le mucléus (Fig. 15). C'est unc cellule encore sins membrane. Ces cellules ou corpuscules ne sont d'ordinaire point sphéripues, mais plus ou moins pyriformes, étant munies d'un prolonsement susceptilsle de s'étirer antificiellement d'me puantité considélable.

Reichert almet que ces cellules sont des l'origino rntomies d'mne membrane susceptible d'ètre détruite très-rapidement par difîsion sous l'action de l'ean pure. Nous ne voulous pas renouveler à ce propos le débal, carr nous aurions les mèmes alrguments à rípriter ici, qur nons avous déji exposés au sujet des cul's ol des zoospermes de l'Asc. Myslax et de l'Asc. suilla. Meissuer serail tres-certainentent ici le l'avis de Reichert, limblis que Bisehoff ne comsentirait a aucum furix à roir la menloratne en litige. Nais les observations de Reichert diffirent des nòtres à est remplie, suivant lui, par des cellules plus gramdes que celles quon trouve plus bas. Il u'a pas en loccasion de rien observer sur le mode de genise de ces dernieres, mais il considire comme raisemblable qu'elles sont engendrées par les premières, dont la taille est double. Tous arons vaimement dherelıe i nous convancre dre l'exactitude de ces dommées; il ne mous a pas été possible de constater me lifférence réelle dans la ggrosseur des cellules des régions indirnées. Aussi, sans vonloir exprimer de doute sur l'existence d'une multiplication par division des cellules on globules dans le teslicule, pensons-nous qu’il

\footnotetext{
'Peut-ètre cette cellule est-elle senle chargée d'engendrel par sa division les cellules qui remplissent le lube générateur. Yous n'avons loutefois ricn observé sur ef sujel.
} 
n’est pas possible de distinguer une région occupée par les cellules-mires plus grandes el une antre par les cellules-filles plus petites. La muliplication qui sopèce ne paraît pas avoir lien far génération endogène.

A mosme que les corpuscules descendent dims le lestienle, l'enveloppe gramuleuse gagne en épaisseur, et les gramules qu'elle contient devientent fulus ívilents (Fiğ. 14). En même temps le nucléole disparPaît, le nucléms devient indistincl, formant mo lache daire an centre de chaque corpuscule sans limites hien détermines. Enfin le moléus disparail complétement sams laissor de trace. Les corpusenles pux-mêmes prennent fiar confre des contours phos décilés; um forme de massue on de poire assez allongér (Fig. 13̈), beancoup plus évilente quanpalrasant. Ils alleignent à ce moment-là une longuent moyenne de $0^{m+011,0 l}$ sont groupés parlaitement régulièrement dans le teślienle, la pointe lournér vers l’axe de lobgane. Les pointes alluèrent, bien que fablement, les mues amx antres. En $111 \mathrm{~mol}$, on a iei une répétition parfaile de l'arrangement des arufs dinss le vilellogène le l'Ascaris Mystax. - Dams lo bas du lesticule les corpuscules pyriformes se séprarno les uns des antres el s'arrondissent en sphires rigulières. Lenr conche externe somble sêtre conlensée mantenant en tune vaie membane. Cepentant il as probahlo que Bischoff ne romdrail voir dims relle ipparente membrane que le contonr d'une houle de sarcode. Les gramulus, jusqualors irrégulièement distribués dams la cellule privio le nucléns, se portent vers la périphérie el sordonneul en rayons aulour d'un centre flus clair, non granuleux (Fig. 16, a). Les cellules sont ici précisément dans un slade de développement analogne à celui que nous avons dejai ru rhez les corpuscules de l'Ascaris suilla, lor'squil so forme chez cenx-di un amas de granules périphériques. Cost le stade que Meissnor lésigne chez les Mermis " chez IAscaris Mystax sous le now te malurité des cellules-germes.

Meisster nomme cet amas maliaire de gramules le moléns de la cellule, mais il ne faul pas le confondre avec le mucléus primilil yui a disparu depuis longlemps. Il n'a rien de commmn arec ec dernier, puisqu’il ros formé par lá condensation les gramules qui étaient 
originairement déposés antour de ce nucléus primitil. Dans cet état, les cellules prolifïrent. Le nucléus se divise, non pas d'après la série binaire habituelle, mais indifféremment en deux (Fig. 16, l, c, d), ou en trois (Fig. 16, e), parfois en quatre. On voit se former d'abord deux ou trois taches claires atl centre de l'amas gr'muleux. Peu ì peu ces laches s'éloignent les unes des imbres, les gramules s'orlonnent en rayons antour de chacune d'elles spécialement. De cette manière se forment plusieurs amas on mucléus a structure layounce. Lal formation de l'amas granulenx radiarre of son partage en phusieurs segments a souvent lien à 111 moment oi la cellule ne s'est pas cucore arondie en sphire, mais conserve encorre la forme ale porro de naguère (Fig. 16, b). Lil cellule s'étrangle antour de chatcm de ces nncléus, at se divise enfin en illtiul de cellules-filles (Fi心. I6, l', g, h) yu’il s'esl lormé de nouveaux moléus. Les cellules-mìres ont rn moyenur un diamètre de $0^{\mathrm{mm}}, 013$; les cellules-filles ont seulement une langent de $0^{\mathrm{mm}, 007}$ is $0 \mathrm{~mm} .008$. L'ilction de l'arile ancélique lail ressorlir, soil whez les unes soil thez les antres, mu nucléte loge an rentro du nuténs, dims la tache claim nou granuleuse (Fig. 17). I d's cellules-filles sont les cellules de développenent des zoospermes.

Les exllules de développement sont à peu pres complétenent renplies firr le nucléus radiairm. Elles słallongent en lorme de poire, tandis que l'arrangenurnl ladiane des gramules disparail (Fig. 18 a). Pendant un cerlinin temps le nucléole est rncore démontrable par l'emploi de lacide acélique. (Fig. 18, h), puis il s’évanouil complétement. Lil queur de la cellule pyriformo s'allonge motablemont (Fig. 19), w la cellule de développement a daus ce mommont (daus le camal déférent) une analogie de torme el dispeet liappantr arec les corpuscules pyrilormes du testicule. De celte forme il n'y a qu'un pas ì celle des corpuscules séminaux lels qu’on les trouve dans la vésicule sćminale. Ceux-ci ont l'apparence d'une cloche surmontéc d'un suspensoir pointu limtiol droit, tantót recoumbé, on colle d'une coupe à boire (Fig. 20). Ils sout mèlés à d'atutres malogues, mais relativement plus longs, plus étroits, tantòt droits, tantòt courbes. Ces derniers resemblent tout à 
lait à une corne de chamois (Fig. 21). Ils représentent un degré plus avancé que les autres diuns le développemont des zoospermes, à savoir le degré le plus avancé quon rencontre jamais dans les organes mâles. Bagge ef Rorichert ont driji distingué deux formes de corpuscules séminaux dans la résicule síminale dı Strongyle anriculane, l'me pyriborme, l'autre cumóiforme. La première paraît ète la forme de notre Fig. 19; la secondp semble correspondre à eolles que nous atrons représentíes dans les fig g. 20 ot 21 . Ces deux auteus remarquent que, sous l'aclion de l'ean pure, la première forme passe instantanément i la seconde. Cependant il est indubitable que ce changement a lien également dams l’intríreur de la vésicule sćminale, indépemlamment de l'action dr l'cau, minjuement en conséquence de l'évolution mẻme du corpusculr simimal. Nous avons dn reste chudir en général les zoospermes dans de l'eau salée. Reichert a dẹjà remarqué avec raison que les zoosprimes prement lous l'aspect cuméilorme dans los organes génitam limelles. Nous summes par contre tris-elisposó a voir une lormation acridentelle. suite dr l'action de l'ean dans l'hémisphère transparent ef incolore quim trouve somvent allhérent a la base du corpuscula campamuliforme (Fig. 20, a). C'est me formalion qui peut rentrer dalls la rafrgorie des expansions de saldeode signales par Biseholf, mais uous noscrions rependant alfirmer qu'olle soil anormale.

Reirhert a distingué daus ee qu’il appelle la tètr du zoosperme me pièce méliane gramuleuse o deux pieces lerninales plus transpareutos. Au centre de la piece médiane so bouve logée, suivant hii, me vésieule qui échate sonvent par l'ellet le la dilfusion. Nous n'avons rien pu constater de semblable, a moins que l'me des pirces terminales plus trauspanente ue coüncide avee cul hómisphère que nous signalions comme expansion de sareode. Quant au corps logé dans la pièce médianc, nous croyons ponvoir affumer qu'il n'existe pas.

Cetle évolution des corpuscules síminaux du Strongylus auricularis est dẹji hien compliquéc, el cependant nous sommes loin de l'avoir parcourne dans toutes ses phases. Néammoins le développenent ultérieu de res corpuscules ayant lieu toujours dams l'intérieur du tube géné- 
rateur lemelle, nous nous aroterons ici dans notre exposition, car nous ne considérons dius ce chipuitro que le développenent des zoospermes chez les mâles.

Si nons passons à l'Ascaris rommulala, nous tromvons que le développement des corpuscules séminims a lien chez elle précisément dlaprès le même schéma. Le lomd du testicule est occupé parr les muclíns, larges d'onviron $0^{\mathrm{mm}}, 006$ ef ormés dhatem d'mu moléole (PI. VII, fig. 2).

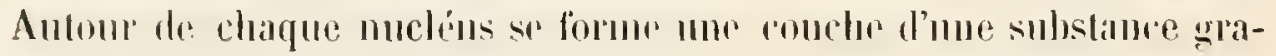
muleuse qui croit par degrés, tambis quo le mucléole, puis le moléns disparaissent, at ainsi de suite. Dans le bas du testionle, les cellulesmòres descellules le déroloppement deszospermes prennent identipuement le mime aspeet que chez lo Strongylus amiculais. Lenr contenu

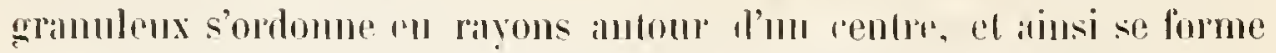

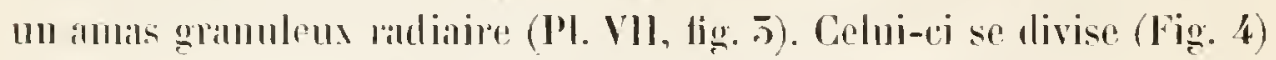
el charm de ses segments devient te mucléns d'une cellule-lille, d'me cellule de dreloppement d'un zonsperme. Les choses se simplitient ici considirablement, paree quil ne so forme jamais alsez l'Ascoris rommutate de zoospermes en lorme de bitomet, de cloche ou de come de chamois, comparables a reux de l'Ascaris Mystax, dr l'Ascaris suilla ei du Strongyhs anricularis. Lal seule molitiention que subissent meore les rellules de développement dans la vésionle séminale du mâle consistr dans la lait qu'elles prenment me apparence plus homogeno, qur leur nucléus disparaîl u que lentrorme peril de sa régularité (PI. VII, lig. 5). Les corpuscules sémintux ont dans cef chat mu diamètre de

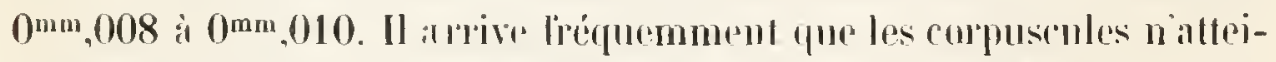
guent pas ce degré do deroloprement dans les organes mîles, mais que les cellules ì mudéus radiatre sont introduites pendint l'ácouplement dans l'utérus de la lemelle, oǹ elles contimuent à se dévelopiner.

Chez lascaris mucromala de la lote, le développentent des corpusculles séminanx est tout parreil. Dans la partio supérienre du testicule, les unclíus, larges de 0 mm, 006 et munis de gros uncléoles, ne sont encore eureloppés que d'une mince couche de sulsstance gramuleuse. Les corpuscules ansi formós adhèrent tous les uns anx antres. Dams le bas du 
lesticule, l'adhérence respective rles corpuscules séminaux cesse. Ceux-ci ont atteint une grosseur considérable $\left(0^{\mathrm{mm}}, 019\right)$; ils sont pressés les uns contre les autres el acquièrent par suite une forme polyédrique (PI. VI, fig. ̈̈, b). Nucléus ot nucléole existent eneore dans leur intérieur. C'est là le degré d'évolution le plus avancé que nous ayons observé chez les corpuscules séminaux de celle espice, de sorte que la forme définitive de ces corpuseules nous est inconnue. Nous avons déjà en l'occasion de dire ailleurs, qu'ancun des individus de cetle espèce que nous avons eus entre les mains n'avait atteint sa maturite sexuelle.

\section{Y. DE LA FECONDATION ET DU DEVELOPPEMENT DES OEUFS.}

Nons arrivons an chapitre le plus important de ce travail, à celui qui ¿ la plus grande portéc physiologique. Inspu’à ces derniers temps le ròle que jonent les zoosprermes dans la fécomlation élail resté un profond mystère. Pour la première hois durant ces dernières années, on a paru aver quelgur cortitude avoir soulevé henrensement un coin du voile. Ce n’est pas que l'essence mème du plrínomine ne soil restée anssi mystripionse qur par In passi; mais la plysiologie a constate un fail importint : la pénćlration du zoosperme dans l'ouf qu’il fíconde. Jusqu'ici cetle furmótration n’a bé observée que rhez un forl petit nombre d'esperes appartrnant, il est via, i divops embranchements du rigne animal. Mais ce momlore est eneore si limite puril ast permis de se demandre si l'on n’a pas tort de vouloir généraliser ces observalions isolies, si la pénétration directe du zonsperme, eonstatée daus cerlains cas, a hicu lien dans tous. Il semble, en effet, fuc dans certaines circonstances une parreille pénétration soil bien difficile. Nous ne roulons pas parter des gros zoospermes des salamandres, parce qu’il n’y a pas entre eux ef les ouls ì fúconder me disproportion qui antorise 


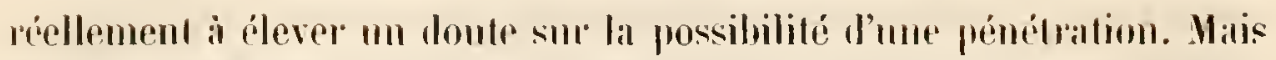
noms citeroms, pall exemple. les zoospermes des Cypris, qui, dijji rela-

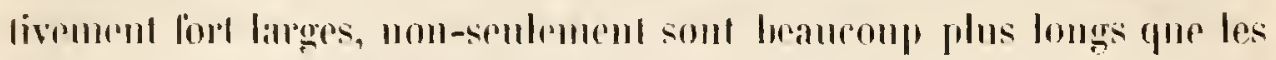

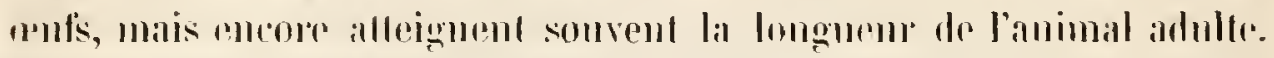

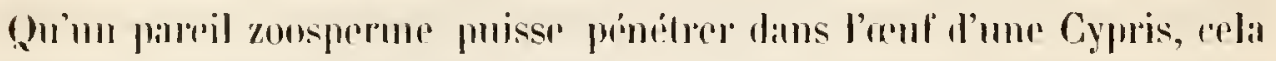

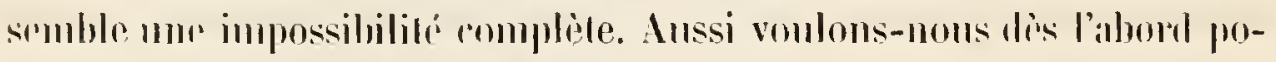

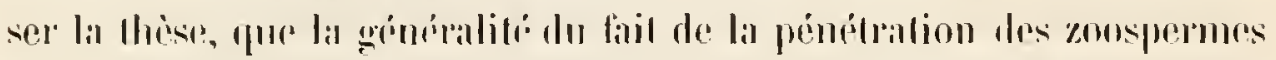
dans l'ipulan moment do la lácondation nest point suffisamment démonlrér. Il est fort pussible pur dams certains cas co soit non pas le

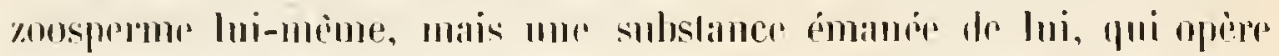
directement la fivondition.

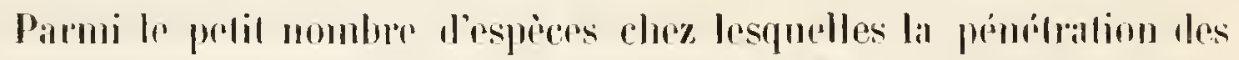

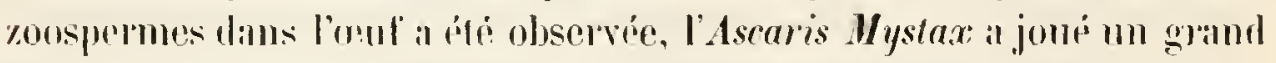

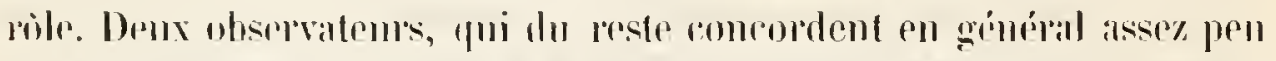
dans les résultals do leurs olsepralions, Nelson el Meissner, ont dieril

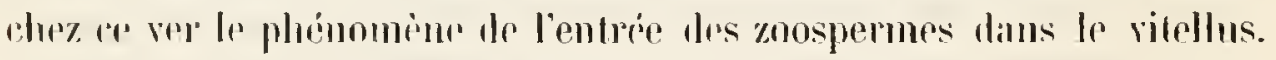

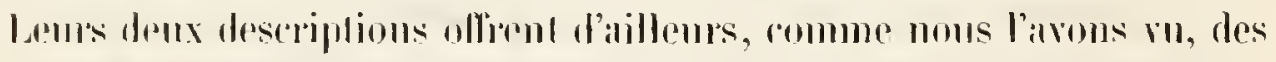
dillibrures notables, mais némmoins les points de rontact soml asse\%

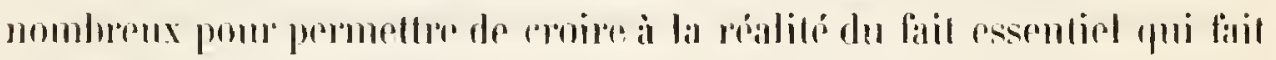
la bitse de cess descriptions.

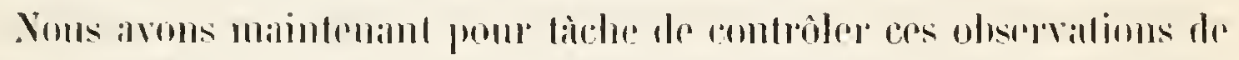

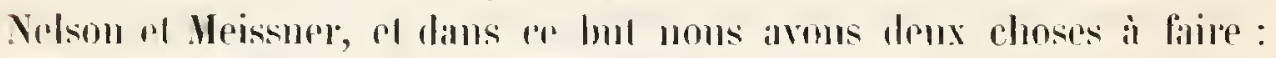

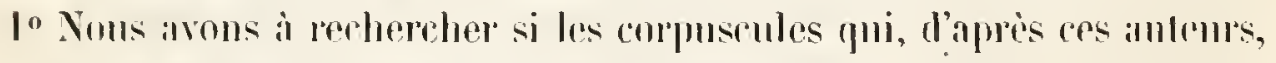

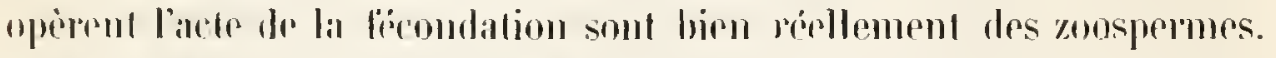

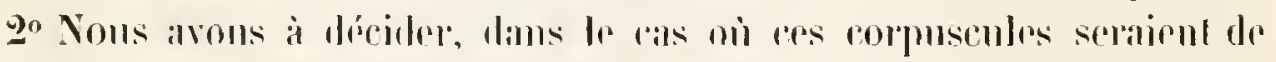
rais zonspromes, si la fícomblalion de lient est hien un résultat de leur prombalion dans lr vilellus, om tout an moins si les obsepvalious dr Nelson o de Heissmer sur re sujel sont dignes le créance.

Noms avons dẹà incliqué fréquemment notre réponse ì la première dre ces questions. Elle usl dr lout point lavorable à Nelson u a Meissner. Reste a lémontrer pourquoi. - Nous avons díji vu que les corpuscules campranulilormes, on, pour mieux dire, en forme de dé ì coudre (c'est l'expression consil'pée), qu'on rencontre dans la trompe de Fallope, 
ne sont pas des producljons épithéliales. Mais de ce résultal négatil il ne

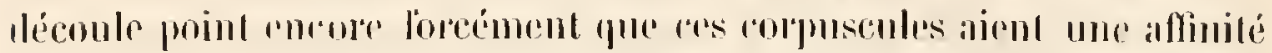

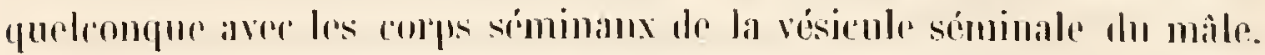

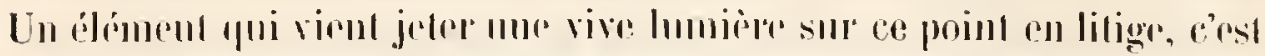

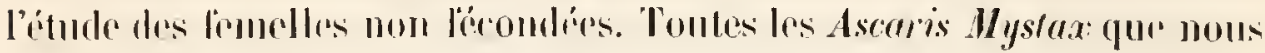

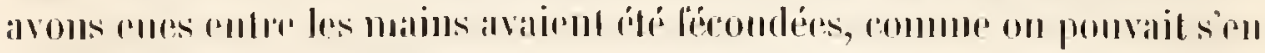

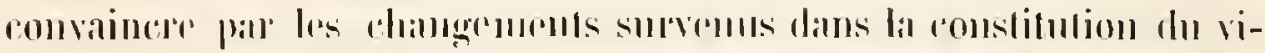

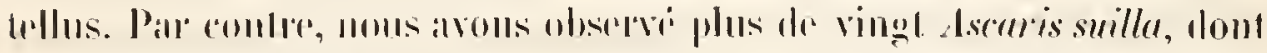

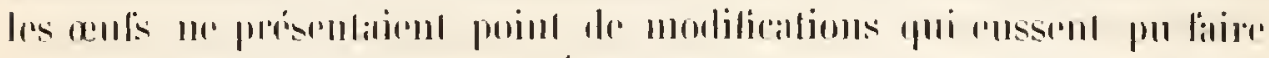

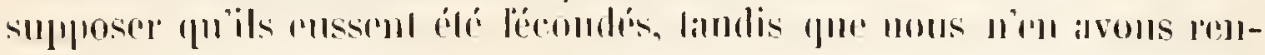

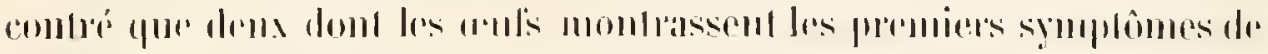

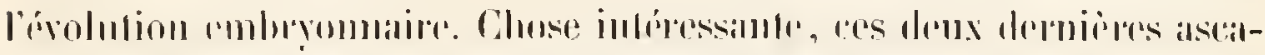

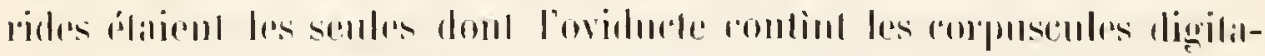

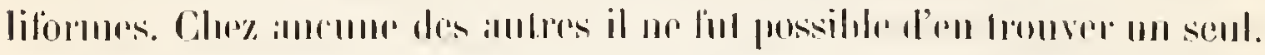

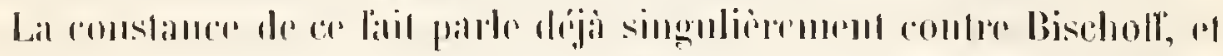

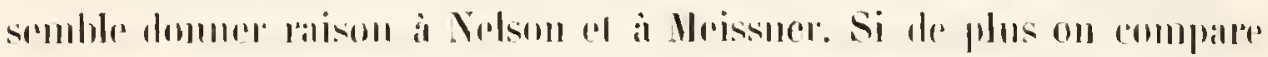

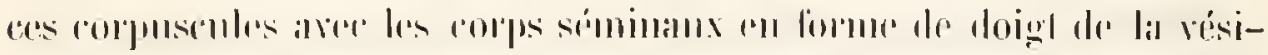

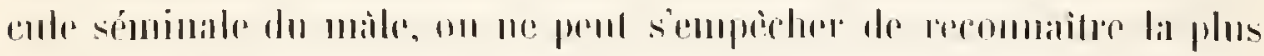

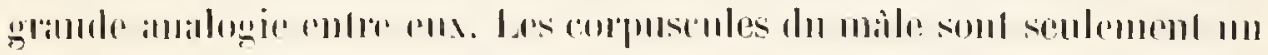
年

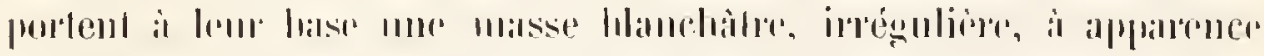

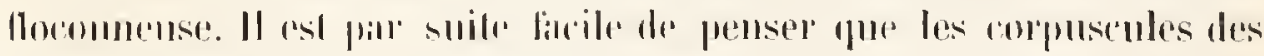

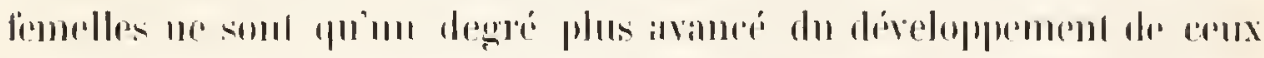

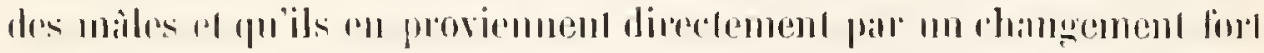

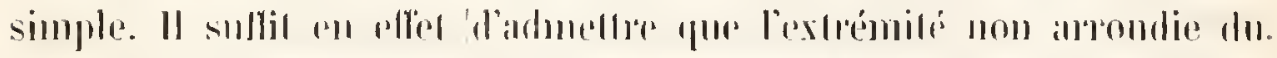

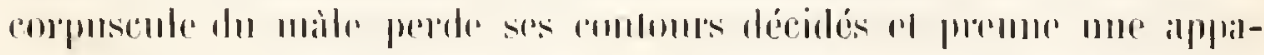

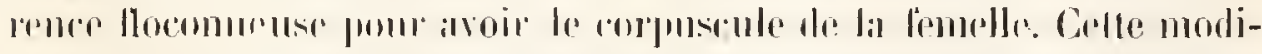

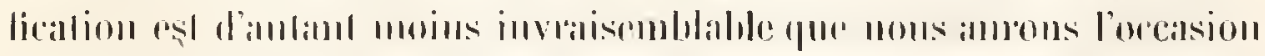

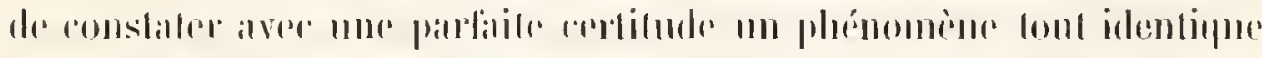

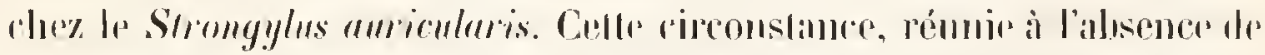

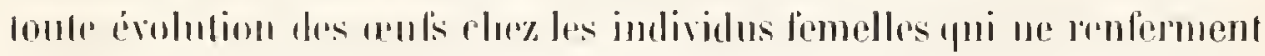

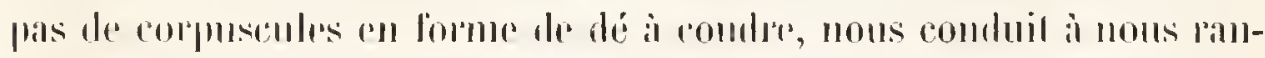

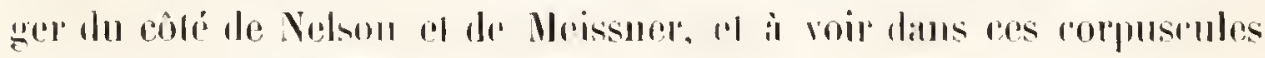
les viais zompermes. 
Nous devons ajouter pue notre ami A. de la V'alelle a lait sul l'Ascaris Mystax lles observations qui concordent parfintement aver celles

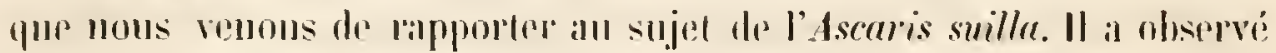

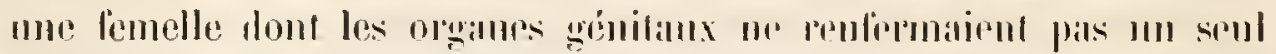

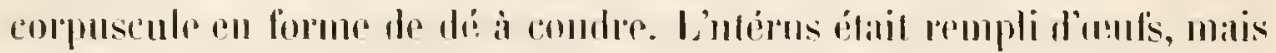

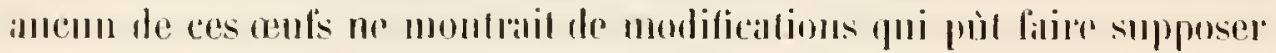

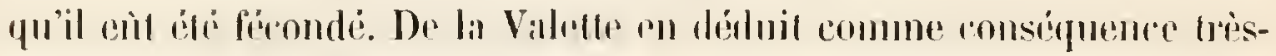

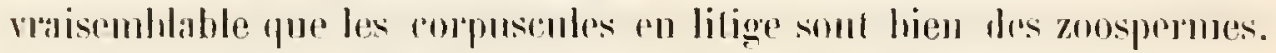

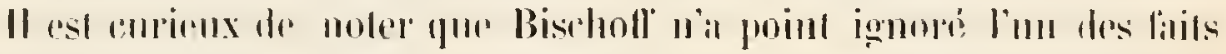

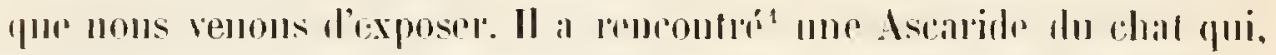

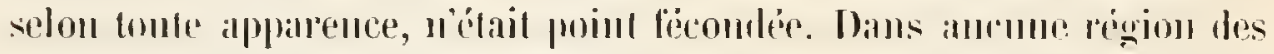

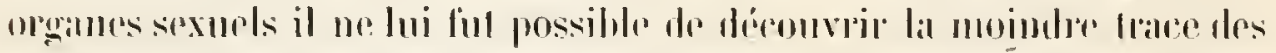

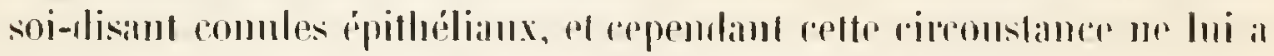

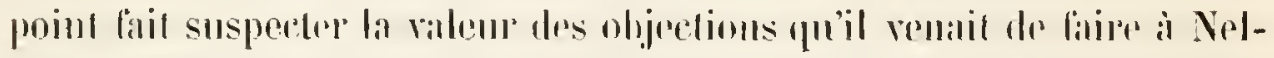

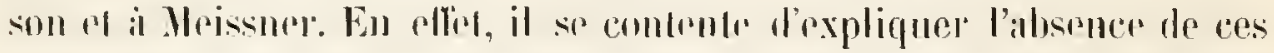

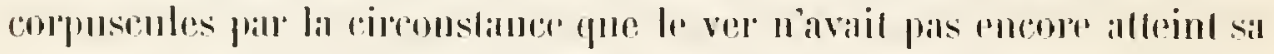

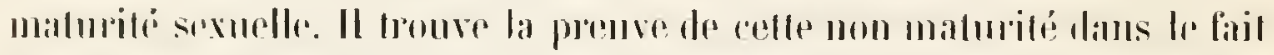

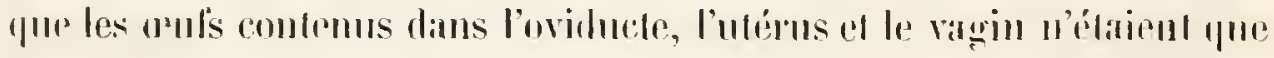

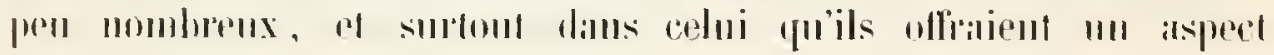

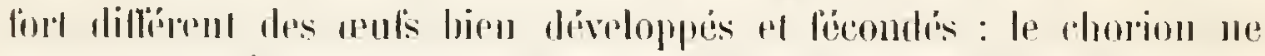

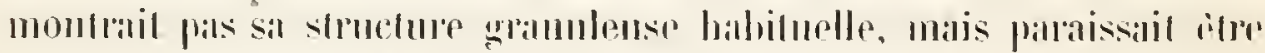

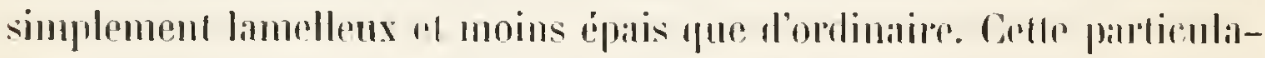

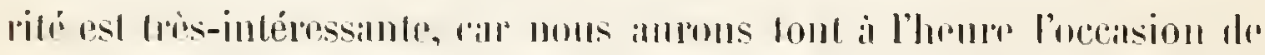
montrep que l’absence drécoudation enstaine la formation d'un cho-

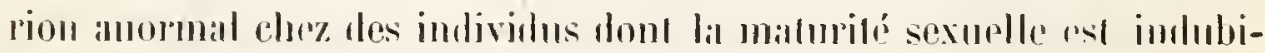
lible.

Les ascarides dh port: non lécondées que nons arons enrs à nohre disposition étaient pour la phupart de grande haille; quelques-mues défassaient ueme notablement le maximum do longuem yu on leur assigne en général. Nous appuyons sur refte circonstince pour montrer que nous riarons pis en a faire à de jemnes individus non encore en

"Ceber Ei- und Samenhildung und Befruchung bei Astaris Mysta L. Lue cil. p. 287-388. 
élal do se reproduine. Soil les oviductes soit les utérus et le vagin,

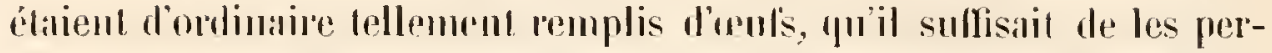

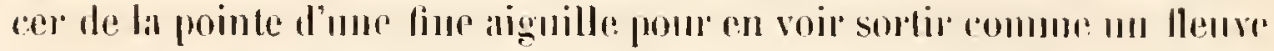

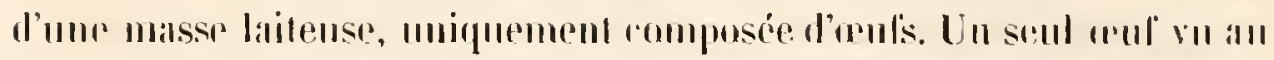
microscope promeltail de déciler arver la plus parlaite rertimbe si lou

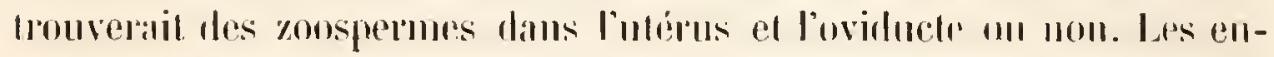

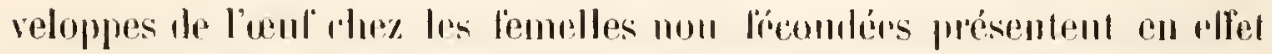

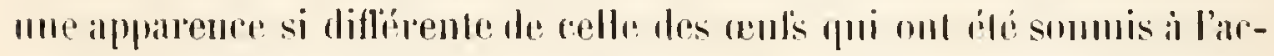

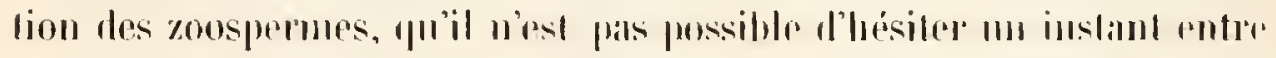

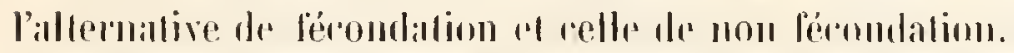

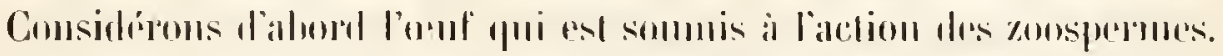

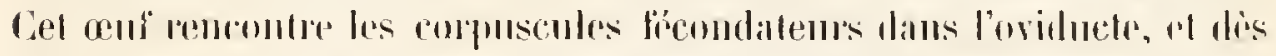
quil a

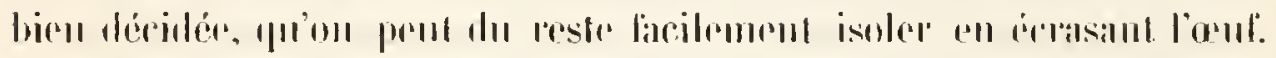

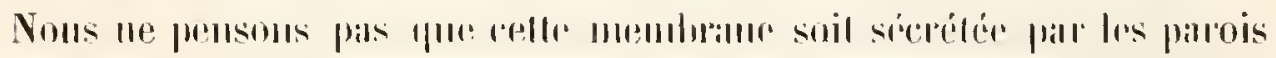

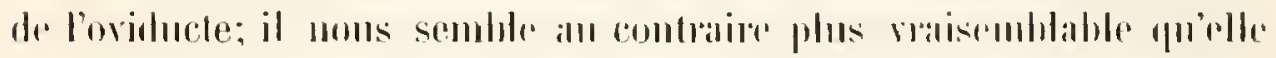

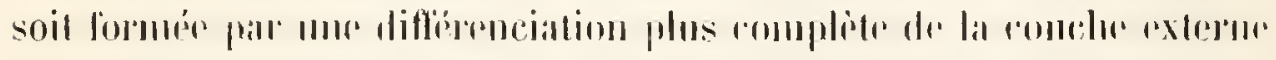

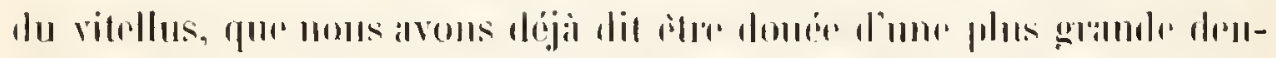

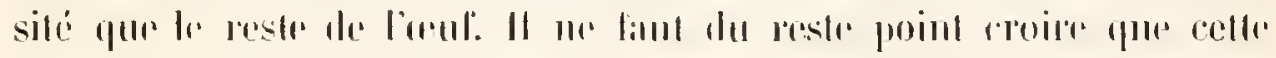
dillérencialion de la membrame soil me romsciquener de l'adion des

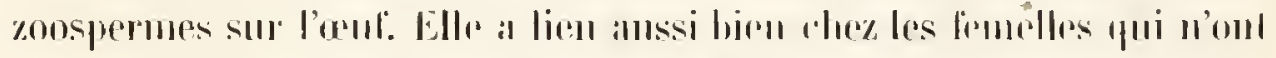

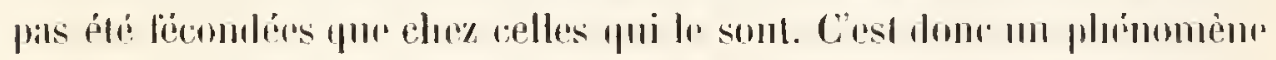

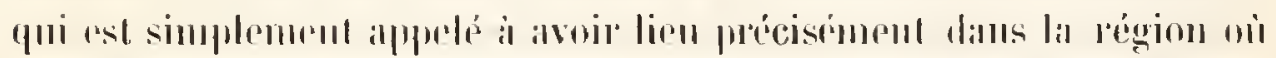

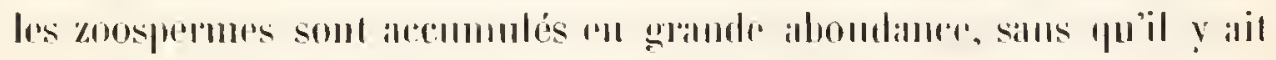

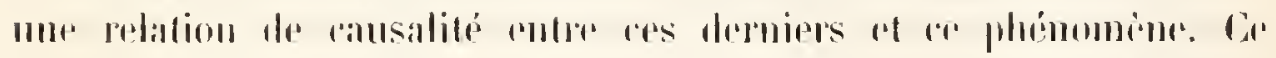

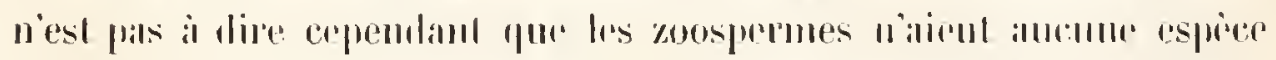

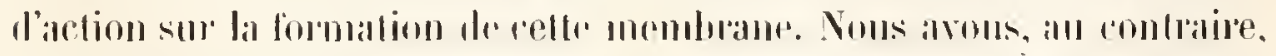

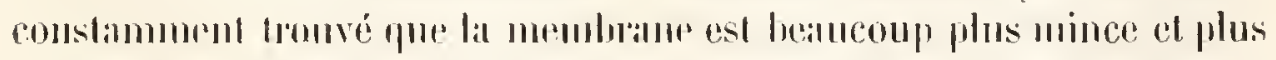
délicate rlum les muls nom fëeondés que chez les aulis lícondés. An-

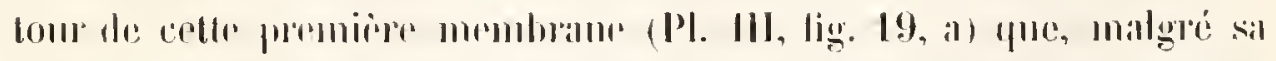
formalion landive, nons droyous ponvoir nommer membrane vilellint: s'en lorme une secomle, lo chorion (Fig. 19, b). Ce chorion est crillen-

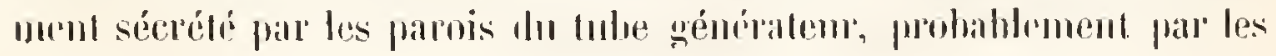


singulieres collules a stolon que nous avoms décrites dams le chapitre scroml. Il alteinl me épaissem asse\% considérable. En mème temps il se produil dans le vitellus mu momement inlime qui indique que l'oul'

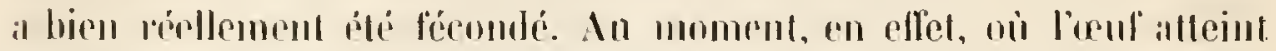

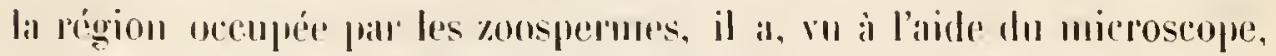
mor roulenr sombre, har a la rébingence inforse des granules vitellins. Anssi rrest-il plus possible de pedrouver dans son intérient la

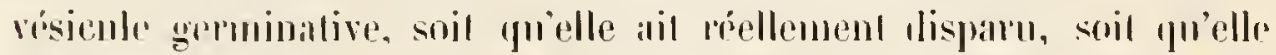
soil trop bien cachée an milien des grammes vilellins. Mlas lamblis que

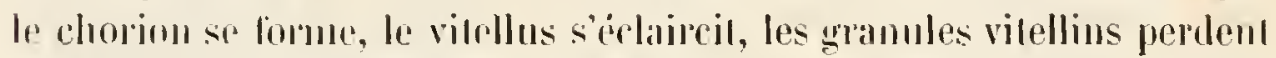

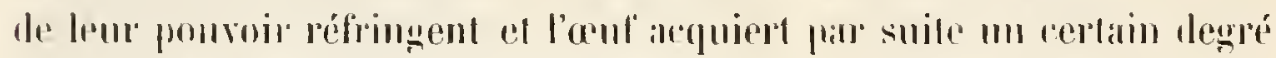

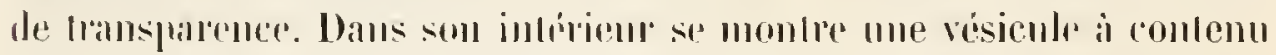

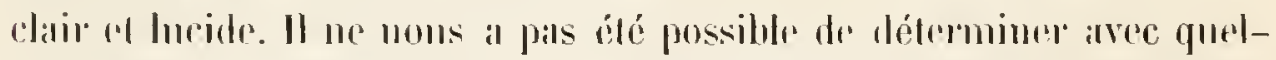

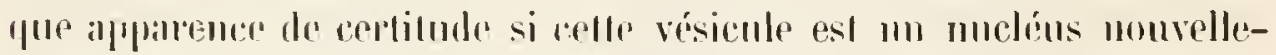
menl lormi, on hien si r'est simplement l'ancienne vésionle germina-

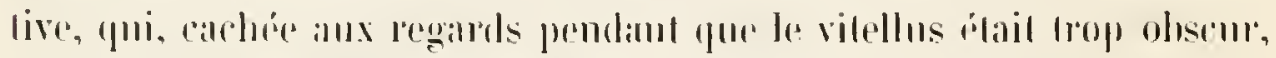

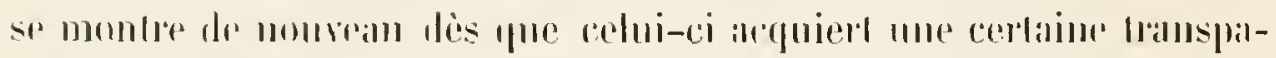

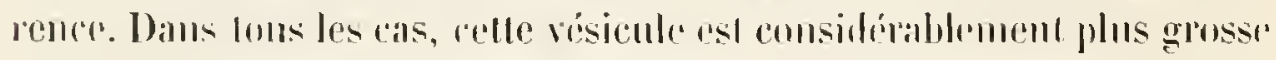

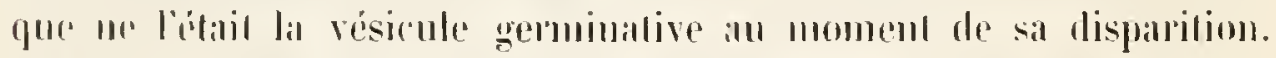

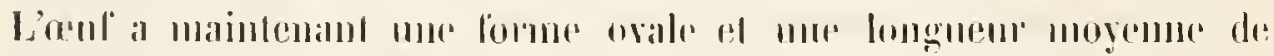

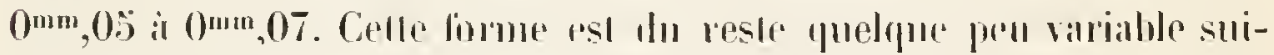
vant les individus, hirn fur frolatement coustante che\% un sent

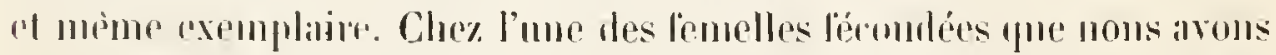

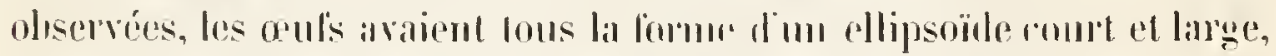

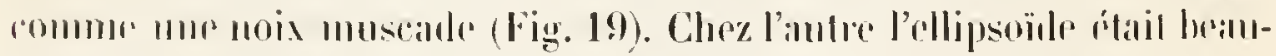

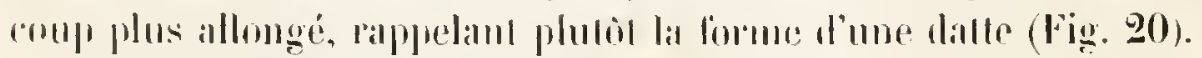

Clez les fomelles nom fécondés, la membrane vitefline se lorme

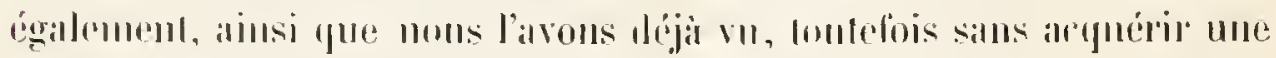

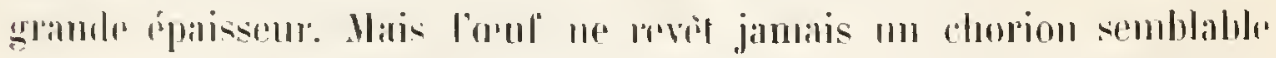

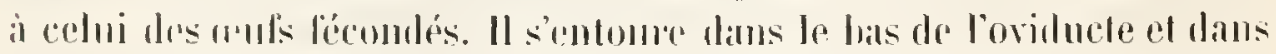

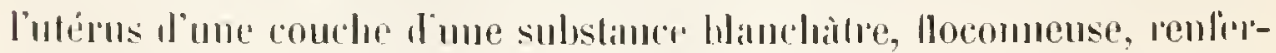
manl da el la de pelits granules lris-réfringents (Fig. 14-18). Cellu

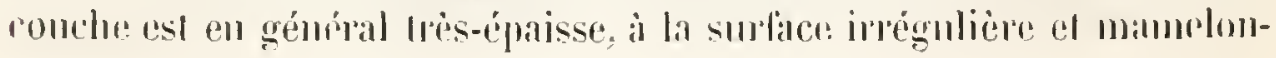


née. Elle est sans nul doute súcrétéc, comme le vari chorion, par les parois du lube génératem, "l l’on trouve cà et lì entre les oul's de grosses masses irrégulières de celle substance blanchâtre. Les espèces d'enfoncements ou de recessus qui existent entre les cellules de l'épithélium sont également fréquemment remplis far une accumulation de celle substance. C'est sins doule la mème sulstance qui lorme le chorion dre l'uul' lécondé, senlement elle ne se rondense pas en me membrane résistante lorsfur la lécomlation n'a pas eu lieu. Les auls des ascandes non lécondées n’ocquièrent du reste point en gémral la lorme régulière el calractéristique des auls aptes à se développer. Un grand nombre d'entre enx conservent me apparence phs on mois irrogulièrentol polyélrique. D’antes prenuent hien mo lorme ovale, mais e'est movale inditerminc, trés-variable suivant les auls quon consildere. Enfin, le vitellus de ces auls ne süclaircil jamais. Il conserve sa couleur sombre, et il n’est pas possible de déconvrir la vésicule germinalive dans som inlérieur. Un grand nombre le ces aul's présentent une série de particularités que nous exposerons plus loin el qui semblent rentrer dans les plénomènes d'une mélammphos rígressive.

Cetle andion des conpuscules licondatenrs, ou pour parler plus exactement, do l'acte de la lícoudation lui-mime sur la formalion des membranes do l'mul' est des plus intéressintes. Un finit du mème ordre est dẹji conmu, mais sur mu intre domaine, smr colni de la holanigur, oi il a élé déconvert en 1853 flar Primgshein. Les spores immohiles des Vaucherial sont originairement dépourvones de membrane dams les spo-

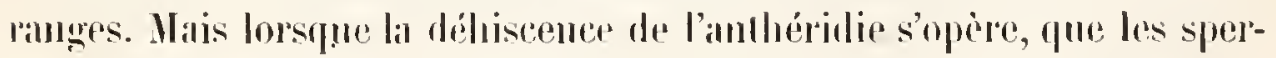
matozoüles ru soptent, et, pénétrant pall le micropyle du sporange, opèrent la lécondiation, la spore lícondér s'entoure rapilenent d'une membrane'. Des olservations toul analogues ont fié faites en 1856 également far Primgsheim sur les spores les Oedognomium².

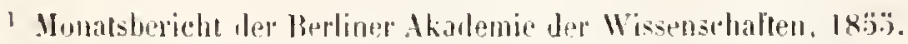

Ibill., 1855. 
C'est sous cettr mênur rublique que doit venir se langer l'observation de Bischoll dajji mentionmér. Le chorion des oul's non fécomlés

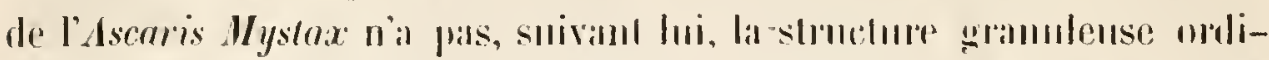

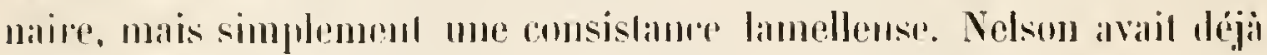

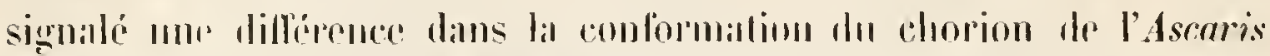

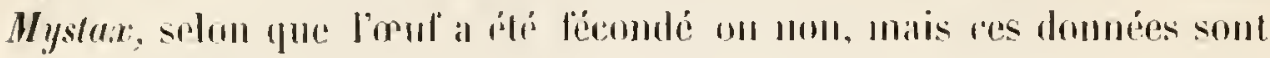

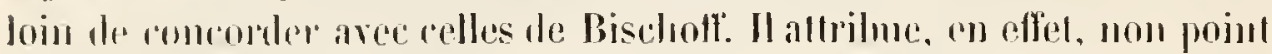

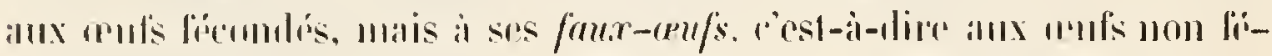

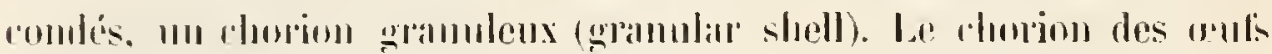

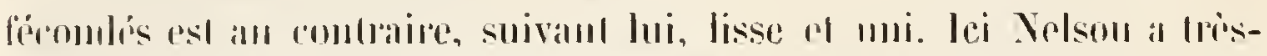
decidrment loul. Toules les Ascaris Wystax que noms arons anes it

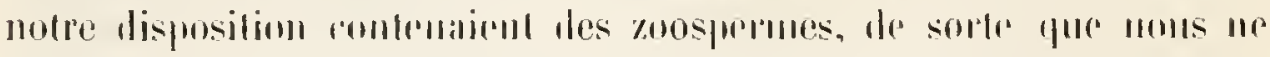

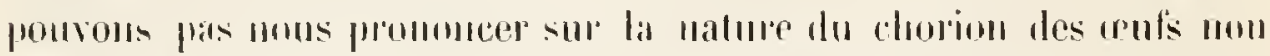

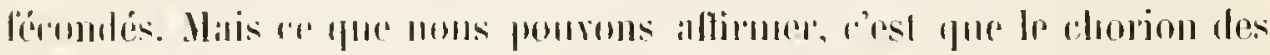

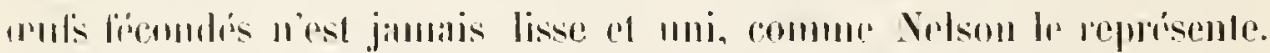

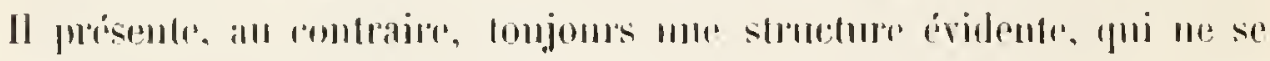

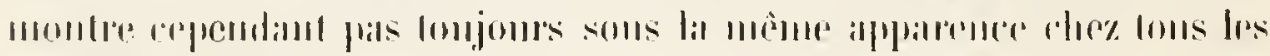

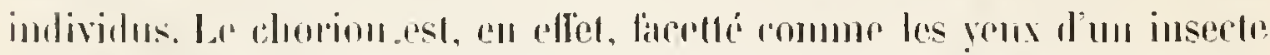

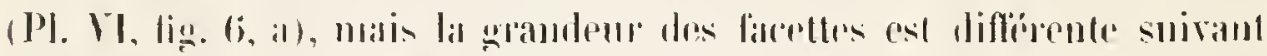

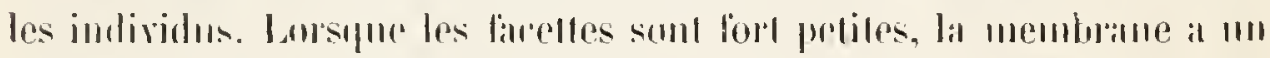

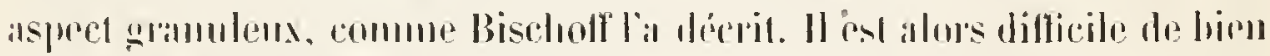

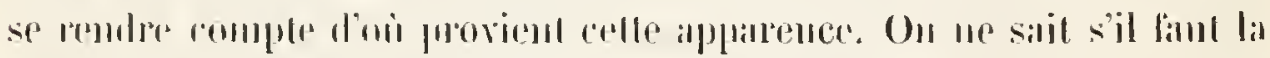

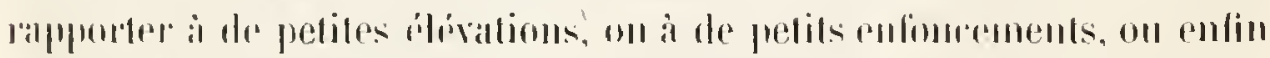

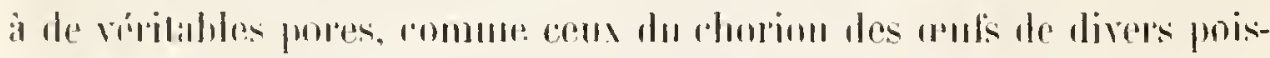

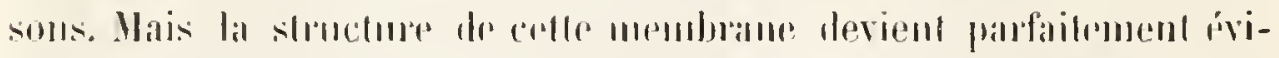

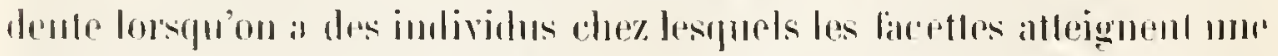

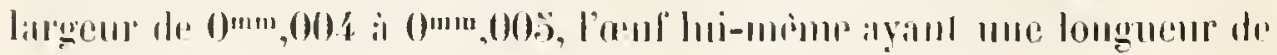

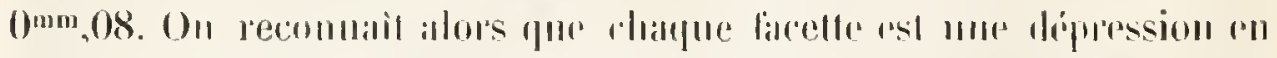

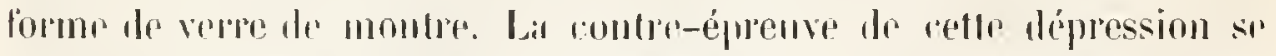

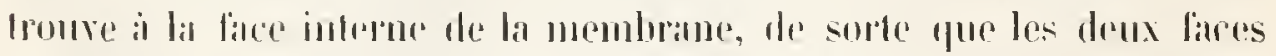

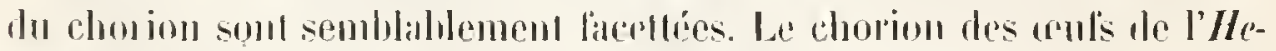
druris amdrophora (PI. VIII, tị̆. 1) prísente anssi une structure qui, all

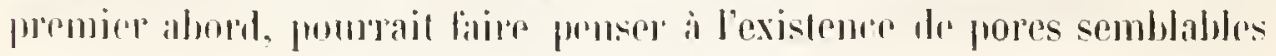


à ceux des oufs de poisson. Mais cette apparence n'est produite que far de petites dépressions de la surface. Ce chorion est relativement l'is-épais, cal les aufs n'étant eux-mèmes longs que de $0^{\mathrm{mm}}$,0今̈, le chorion a une épaisseur de $0^{\mathrm{mm}}, 005$. Il est mumi d'une onverture à claque pòle de l'aul, ouverture qui paraì n'ètrér fermće que par la membrane vitelline. La grande épaisseur de ce chorion permel de s’assurer avec certibule qu'il n'existe pas de camiux traversinl la membrame de part en part.

Il ne laut pas croire do reste que l'acte de la lécondaltion oprive parlout sur la formation a chorion ume achion amalogne ì celle que nous venons de voil chez l'Asearis suilla el l'Ascaris Mystax. On n'observe, pill exemple, rien de semblable chez l'Oxyur is vermicularis de l'homme. L'aul', qui dans l'uraire a la limme d'mu disque tres-mince, preml dans

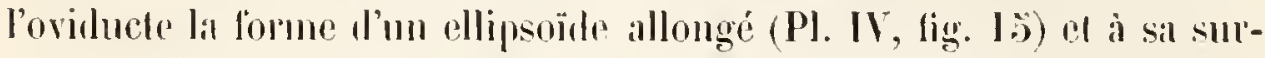
lace se dillérencie une membrane vilelline nes-apaisse. Puis se lomme

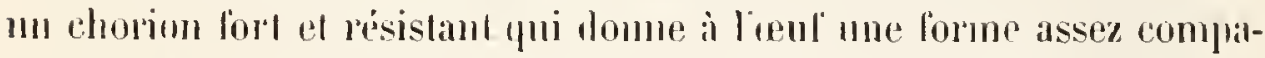
rable a celle d’une whaloupe prontée. C'est un ovoüle aplati en long sur l'un des cotés (Pl. IV, fig. 16 et 17). Ce chorion est tres-fragile. Il se Lrise fréquemment en travers lorsqu'on exerce une légère pression sur la plique de reme qui recourre lobjed (PI. IV, fig. 19). Soms l'action te l'acide acélique, il se distend considérahlement el acquient une amplem trois on quathr fois anssi considérable que celle de l'reul'. Lal constilution de re chorion esl pandiatement identipue chez les ants lécondés et chez cenx qui me le sont pas. Il est meimmoins lacile le savoir dis le premier coup d'ail si l'on a a lirire à une fomelle fécondée on non. Chez les lemelles fécondées, les ntérus sont remplis de milliers d'aufs, dont chacun renferme $11 n$ embryon déjà tout formí. La lace ventale du ver el sil quene (Pl. IV, fig. 17 et 18 a) regardent sams exception du còté aplati de l'neuf. L'embryon est fort large de corps el remplit lont l'espace intríieur. Un embryon tel que celui que kïchemmeisler a représenté ' sous la forme l'un petil ver filiforme, conlourné sur

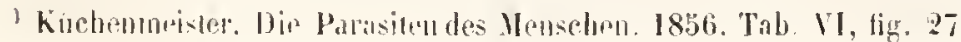


lui-mème et n’oceupant qu'une très-petite partie de la cavité de l'oul, ne se présente jamais. Chez les femelles non fécondies, au contraire, les utérus sont remplis d'oufs qui, an lien d'embryon, renfernent un vitellus non seg̨menté muni d'une grosse vésicule germinative (Pl. IV, fig. I6). Cette vésicule u’es pas visible aussi longtemps que les oufs ont la forme de minces disques; elle ne se montre que lorsque l'ouf conmence i prendre une forme ellipsoide dans l'oviducte. Il est cependant probable que cette vésicule est la mème qui étail originairement visible dans le blastogine.

Nous ne savons si les appendices dont est muni le chorion des rufs chez les Gordius, l'Ascaris dentata et divers autres nématodes se forment par suite de la fécondation, ou indépendamment de celle-ci. Nous arons tentẻ d’étudier au point de rue de l'action de la fécondation m chorion a slruclure fort singulière, i savoir celui des aufs de l'Oxynris spirotheca yu'on rencontre dans l'intestin de l'Hydrophilus piceus. Ce chorion a une structure tout i fail comparable is celle d'une traclice d’insecte (Pl. VIII, fig. 9 et I0). C'est une euveloppe composée d'un seul fil emroulé en spirale. Ce fil se laisse dévider précisément comme celui d'une trachée, et donne les réactions de la chitine. Nous novons flas réussi à recomnaitre la manière dont le fil se forme. Il est probable cependant que les parois le loviducte sécrètent la substance du chorion, el que le fil ne se forme que secondairement par une scission spirale de lit farsse-membrane sícrilée. Parfois le fil passe d'un reuf i l'autre de manière à ce que le chorion des leux aufs soit formé par un seul et mème filameut spiral. Mais ce n'est point la règle. C'est probablement un cas qui ne se présente que lorsque deux oufs sont accolés l'un à l’autre par la substance, sins doute originairement glutineuse du chorion. Nous ne sommes malheureusement arrivé à aucun résultat relativement au rôle que l'acte de la fécondation peut ètre appelé à jouer dans la formation de ce singulier chorion.

Malgré l'aboudance de ce parasite, nons n’arons jamais eu la chance de trouver un seul mile, fait qui s'explique soit par la rareté réelle, soil par la petitesse probable des individus du sexe maseulin. Parmi 
les nombreuses femclles que nous avons observées, les mnes étaient encore jeunes et non fécondées. Leurs oufs n’avaient pas de chorion, mais comme ces oufs citaient encore dans la partie supérieure de l'oviducte ef que pas mn seul n'avait atteint l'utérus, il n’est pas inupossible que refle absence de chorion doive être mise sur le comple de la jeunesse de l'animal el pas sur celui de l'absence de fúcondation. Les autres lemelles étaient adultes, mais toutes ćtaient fécondées. - Les fails que nous vemons de rapporter sur les aufs de celle Oxyuris nous sont connus depuis le printemps de 1855. Györy', qui dans l'intervalle a décrit l'animal sous le nom d'Oxyuris spirotheca, a également signalé celte singulière strncture de l'enveloppe externe rle l'ouf.

Nous nous sommes efforcri avee beancoup de soins d'observer chez divers nématodes le mode suivant lequel la fécondation des couĺs s'opère. Mais nous ne sommes arrivé à ancun résultat. Nous n’avons rien vu fui phit nous laire présumer avec quelque vaisemblance que les zonspermes pínètrent daus le vitellus. Nous savons qu’une observalion mérative ne peut jamais en renverser une posilive, anssi nous garderons-nous d'arguer de ce lail que Nelson et Meissmer soient arrivés à les résultats erronŕs. - Cependant il nous est permis de venir éclairer dn llambeau de la eritique les domées le ces denx observateurs, de peser mìrement jusqu’i quel point elles somt dignes de foi et jusqu’ì quel point elles faiblissent levant un cxamen raisonné. Nous aimons it plarer dles points de donte devant toutes les observations capilalus anxipuelles les preures lont dílint, et nous ne croyous pas que re soil un mal.

Et d'ahord, ì supposer que les zoospermes rampaniformes pénètrent bien réellement dams le vitellus, il s'agit rncore de décider s'ils y pénètrent do la lacon que décrit Nelson on de la maniere quindique Mrissnrr. Nelson a hourí des zoospermes en grand nombre appliqués contre les orules. Nous le croyons sans peine. Ces corpuseules ont la propriété d'athérer facilement anx objets étrangers an moyen de leur

${ }^{1}$ Gÿ̈ry. l.oe. cil, 11. 327. 
parlie floconneuse. C'est même là ee qui explique l'erreur de Bischoft, de Leuckirt el d'Eekhard, qui, voyant quelpues zoospermes aldhérer a la paroi de l'ovaire, ont roulu firro de ees zoospermes une partie intígrante de cette parni. Mais Nelson va plus lon. Il a vu les zoospermes déprimant la surface des ovules, cenx-ci se déchirant irrégulièrement i leur surface et admellant les zoospermes dans leur intérieur. Nous crogons certainement à l'exaclitude de la deseription el des figures do Nelson. Reste ì savoir seulement s’il a eu ì faire à un phénomène normal ou purement artifieiel. Lorsque nous considérons les planches de Nelson, nous ne prowons nous défendre de pencher phutot vers la seconde alternative. Nous n’avons jamais vn dans la partic de loviducte oì la fëeondalion s'opère les aufs prendre une forme scmblable à relle que Nelson représente. Nous ne powrons reconuaitre dins la figure donnóp par ce sivant que des oufs éerasés. Que, par aventure, des zoospermess pénètrent dans le vitellus par suile de l'écrasement de celui-ci, e'est chose facile à comprendre, mais c'est naturellement un événement tout fortuit. Thompson a été plus prublent que son ami à l'égard de la question de la fécondation. Il reconnait l'exactitude des figures re Yelson. II a vu les zoospermes s'attacher aux reufs par un point quelconque de lenr surface, par le còté, ou par la partie arrondie aussi lien que par li bise floconneuse; il a vu aussi les déchirures les anls, mais il n'ose alfirmer avoir bien réellement ru des zoospermes dans l’intérieur du vitellus. Il doule mème qu’on puisse consilérer ces phénomènes conme des circonstances qui accompagnent nécessairement la fécontation. D’après lat deseription soit de Thompson soit de Nelson, il nous semble que ces observatenrs se soient contentés l'observer an travers des parois de l'oviducte. C'est là un mode d'observalion qui n'est certes pas à négliger, pour s'assurer de la position respective des parties. Mais il est évident que ce n'est pas non plus le seul auquel on doive avoir recours. Le peu de transparence de l'ohjet nécessite en effet une compression assez forte, font le résultat immédiat est la déformation des aufs. Lor'squ'on lend les parois de l'oviducte et laisse les oul's s'échapper librement soit tlans l'eau pure, soit dans 
de l'eau légèrement salée, on ne rencontre jamais de ces oufs déchirés dont parlent Nelson et Thompson. On s'apercoit aussi alors que l'adhésion des zoospermes aux oufs n'est point aussi fréquente que ces anteurs le prétentent. Nous doutons en particulier beaucoup que les zoospermes puissent alhérer normalement aux oufs par une autre parlie que leur partic llocomeuse, bien que Thompson affirme positivement le contraire.

Nous nous rangeons donc du còté de Meissner pour contester à Nelson l'exactitude de sa lhéorie sur la pénétration des zoospermes tans l'cut. Yoyous maintenant jusqu’à puel point la théorie de Meissner hiimême mérite plus de confiance.

Nous avons déji montré qu’il n’existe pas de micropyle dans le sens the Meissner. Mais cela ne suffit point à démontrer que les zoospermes ne prínètrent point dans le vitellus, précisément a la place où ce sivant admet l'rexistente d'une onverture de sal mombrane vilelline. Les zoosprimes, dit Meissner, adhirent beanconp plus sonvent à la région nifropyliemne que partoul ailleurs, el il lui semble probable que cetle allıésion est facilitée par la mrmbrame qui enveloppe le zoosperme. Meissner a donne, en effet, me description de la formation des zoospermes qui lilliere notablement de la notre. Le zoosperme se díveloppe, suivant lui, dams l’infériur de la cellule de développement; il devient phus long qu'cllo, tellement quili est obligé de se courber dans son intérienr; puis tout d’un eonp il se redressa en déchirant la membrane an point qui correspond à l'extrómité floconneuse. Mais la mombrane most point détruite pour ala. Elle sulsiste, sclon Meissner, conme une roille sur le corpusenle en lorme de cloche. Cepentant nous n’avoms su trouver ni che\% l'Asraris sulla ni chez l'Ascaris Mystax trace de cetle coille. Le ropps lui-mine du zoosperme avait une apparence asser lomogène, et réfructait notahlement la lumiore; an centre do l'extrimité flocommense se tronvait le plus sonvent le granule que Nelson "t Meissner considprent comme le nucléole de la cellule de développement (P. V, ligg. 10 o I1, al). Mais de mmuluane enveloppante, point. Une senle fois chez l'Ascaris Mystax parmi des milliers de zoospermes 
nous en avous tomé un qui repondait parfaitement anx ligures de Meissner (Pl. V, fig. 11, A). Mais nous ne pomons considérer ce fait que comme une exception.

Sans vouloil nier précisément l'exactitude des fignres dans lestuelles Meissner représente mu zoosperme adhórant à sou soi-disint micropyle (il n'y a, en eflet, ripn d’uvaisemblable a ce que les zoospermes arrivent parfois en contant arre cette place de l'ouf comme avec toute autre, el y athèrent légripenent), nous devous dim que nous n’ams jamais rien vu de semblible, mais que, pal contre, nous avons fréquemment observé chry l'Ascaris sailla mo phénomène qui permet pent-étre d’intrepréter tont antrement les ligmes en question. On rencontre trèssomvent dans l'oviducte des femelles non fícondies des oufs non encore arrondis, mais déjì pourvus d'une membrane distincte, aufs dont l'apex est considérablement allongé (PI. III, fig. T). Un tel exul ressemble font a fait anx unfs que Meissuer a figurés avec un zoosperme placé sur le micropyle. Parfors le vitellus se retire légèrement de la menbrane dans le prolongement apicial, et celui-ci y gagne une analogie de forme d'autant plus grande avee les zoospermes de Meissner revelus de leur coille (Pl. III, lig. 8 et 9). Cependant il est rertinin que cet apex n'est point un zoosperme adhérent à l'wenf, car on rencontre des aufs dans ret état chez des femelles dont les organes génitaux ne renferment du reste pas un seul zoosperme. La poursnite du phinomíne montre d'aillenrs qu'il s'agit ici de l'exclusion d'un fragment du vitellus. Liapex se sépire de l'ouf proprement dit par un étranglement pui devient de phus en phrs profond, si hien que le fragment de vitellus linit par ne plus adliérer ì l'ouf' que par un isthme tubuleux formé par la memlurane vitelline (Fig. 10). Il a en général alors me forme ovoüde, ef comme il est revêtu par un fragment de la membame vitellime, on croirait volontiers avoir sous les yeux mo oeul lilliputien adhérent à l'uruf normal (Fig. 11). Dans cet état, l'oul' el soll appendice oroüle s'ontomrent le plus souvent simultimément du fant chorion des aufs non féroudés. Puis listhme tubuleux qui s’est étranglé tonjours darintage, finit par se rompre, et le corpuscule ovö̈le se sépare de l'ouf 
(Fig. 12). Voili pomrunoi l'on trouve dans l'utérus parfois en assez graude abondance, outre des oufs normaux, des corpuseules de petite taille (Fig. 15), rappelant tout à fait les véritibles oeufs par leur apparence extérieure, puis(puils sont formés d'un vilellus, d'une membrame vitellime et d'un finu chorion. Nous ne prétentons point alfirmer que Meissmer ait en sous les yeux les images que nous venons de décrire, mais la chose n'est pas impossible. Nous ne mentionnons dont ce fait que parce quil est pent-ètre une des circonstances qui ont poussi Meissner à admettre son micropyle. Cettr supposilion est d'autant plus vaisemblable que Heissner rapporte avoir trouvé des oent's dans lrsquels un zoospertme avail pénćtré en laissant sa coiffe posée comme une cloche sur le micropyle. Lil coiffe du zoosperme n'existant pas, selon nous, la membrane vue par Meissner ne peut pas avoir appartenu au zoospernur. Il est forl probable que c'átait simplement la pantio de la membrane vitelline qui revit l'apex. - Celte expulsion d'un fiagment du vilellus n'est du reste point un filit nouveiu dans la science. Nous en comnaissons des exemples nombreux décrits en particulier par F. Müller, van Beneden, Lovén, Koren et Danielssen. La vósicule directrice de F. Müller n’est, en cellet, pas autre chose qu'un tel fragment de vitellus. Sans vouloir entrer dans ume discussion de la théorie émise par Mïller sur le ròle que jour re fragnent de vitellus relativement à la segmentalion de l'uuf, nous devons dire cependant que dans le cas spécial qui nous occupe il ne peut pas ctre question d'ume relation intime entre l'exchusion de ce fragment ef la segmentation le l'oenf. En premier lieu, en efiet, l'expulsion du fragment de vitollus n'est point un phénomène général qui se présente chez chaque oeul' do l'Ascaris suilla. En second lieu, nous ne l'avons constaté précisćment que chez des femelles non lécondées, chez lesquolles il n'ptait pas question de segmentatiou des oeuts.

Le point capital dans cette critique est celui qui concerne la présence de zoosprermes dans l'intérieur des oufs. Meissner affirme avoir vu les corpuscules campanuliformes dans le vitellus lui-mème, en dedans de la membrane vitelline. C'est une affirmation que nous n'avous pas le 
droit de rontester, el si les aufs dans Iesqunels Meissner a observé des corpuscules síminaux u'offraient pas de déchirme arcidentelle, c'est là une prenve incontestable que les zoospermes pénètrent dans le vitellus d'une manicire on d'me antre. Nons n’avons malhemensement pas été dams le cas de rruncontrer noms-mèmes des aeuls qui présentassent rien le sembliblo.

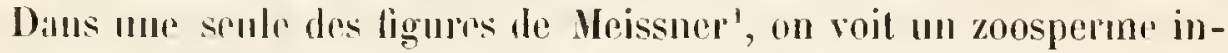
rontestahle daus l'intérient de l'ouf. Dans le texte cependant l'auteur dit yu'il a trouvé partois trois on quatre zouspermes daus le même auf clury l'Ascaris Myslax, of que depuis lors il s'est convaincu, primcipalement chez J'Ascaris megalocephala, que d'ordinaire phusieurs zoosper-

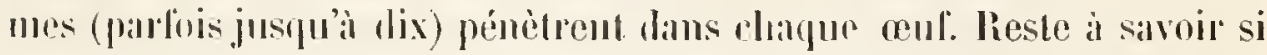
refte alfirmation so hase réellement sur lobservation de zoospermes incontestables dans lantéricur des aeufs ou simplentent sum la rencontre des produits de leur mélanorphose ultérieure. Si celte derniere supposition est racte, ef nons le croyons volontiers, la démonstration de la pruébalion de phusionrs zoospremes repose, comme nous allons le voir, sur des bases bien chancelantes.

Soit Nelson soit Meissner ont vu les zonspermes me fois amivés daus l’intŕlien des aufs subir des modilications nolables. Nelson les a vas perdor leur forme caractéristipne, se luméficr, se transformer enfin en masses irregulieres, tramsparentes of fortemrnt rélingentes. Meissner, de son côté, rantène ces modifications à ume transformation graduelle en graisse. Le contour de la partic campanulilonme du zoosprerme se dessine, d'après sa lescription, tomjours plus neltement, tout en devenant phus sombre ef en acquérant mo érlat particulier. Cette partic en forme de cloche devient plus rofringentre s'arrondit de plus en plus, ef rulin il n'est plus possible d’y lrecommaître antro close quime

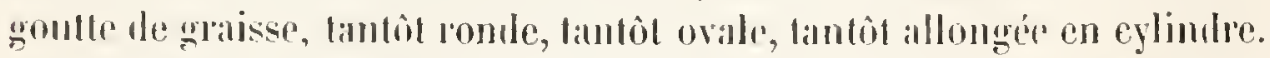

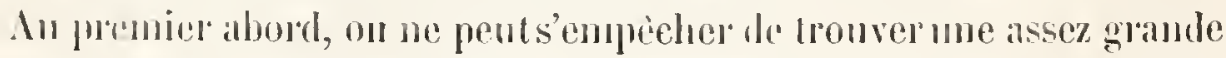
analogie entre les deseriplions des deux antenrs, analogie qui somble

\footnotetext{
1 Heobachungen über das Eindringen der Samenelemente in das Ei. Loc. cil. Fig. 6, b.
} 
parler d'aulant plus en faveur de la justesse de l'ohservation que ces auleurs ne sont pas très-souvent d'accord. Cependant un examen plus attentif montre que l’acrord de Nelson ef le Meissner n'est ici qu'apparent. Nelson, on s'en souvient, a armis qu'm certain nomlme d'oufs échappent à la fécomlation; ce sont ses faux aufs (false ovum). Ces faux oufs ne tarlent pas, suivant Nelson, à subir nue espècr de métamorphose régressive. Une fois le chorion formé, leur vísicule el leur lache germinatives disparaissent, et à la place de celles-ci se montrent un certain nombre de gros globules transparents, ressemblant beancoup phus à des goulles d’huile qu’à des aggloméralions de cellules. Nelson pense que ces globules se forment en conséfuence, d'un part de la disparition do la vésicule gemminative, el l'autre part l'une séparalion qui s'effectue entre l'huile et les gramules du vilollus. Les corpuscules résultés de la mélamorphose des zoospermes ne peuvent se confomlde, dit Nelson, avec ces goutles d'huile, attendu qu'ils ne présentent jamais la régularifi de contour des gouttes huileuses des fiux oufs.

Maintrumt on recomaitra sans peine que les gouttes d'huile des fanx ouls de Nelson ont une beatcoup plus grande analogic aver les goulles de graisse de Meissner, que les rorpuscules réfrimgents prouluits, snivant l'analomiste anglais, par la métamorphose des zoospermes. C'est ce qu'a compris Meissuer; anssi refuse-t-il de donmer anx false eggs de Nolson la mème siguification qur celui-ci. Ce sont, à ses yeux, des auts qui ont éti bel et bien fécondés, et les gontles d'huile sont des zoospremes en voie de subir la mítamorphose graissense.

De toutes ces domnées contradictoires il n'y a quo celles de Nolson relatives a ses faux oufs que nous soyons en itat de confirmer d'une manière posilive. Si les allimalions de Meissner reposaienl sur une hase réelle, il landrait que chaque oul ou presque chaque ouf contint, lorsqu'il a atleint le bas de l'oviducte, me ou plusieurs gouttes d'hnile. Mais ce n'est point li le cas. Ies oufs qui renferment de semblables gontles ne forment que l'exception chez les femelles fécondées. -- Cliez les femelles non fécondées de l'Ascuris suilla, par contre, on peut étudier le phénomine de la formation des gontles d'lucile sur une bien plus 


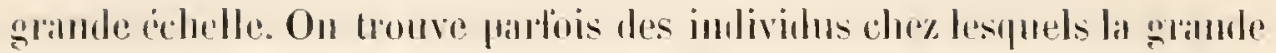

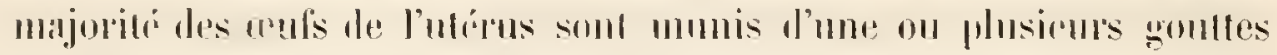

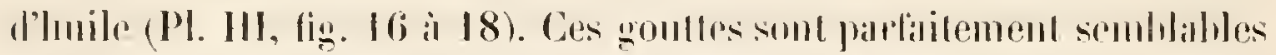

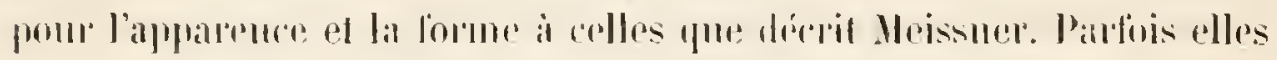

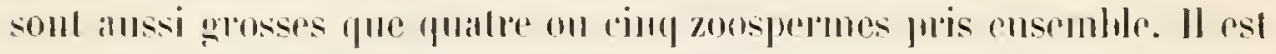

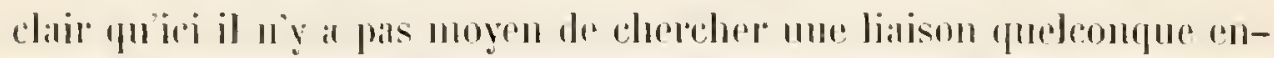

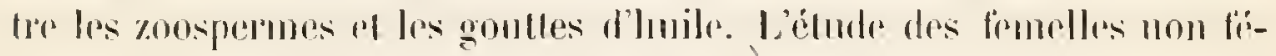

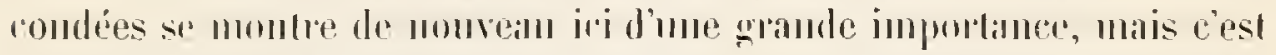

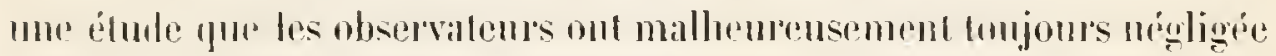
jusqüici. - Nous considerons la formation des gonlles d'huila comme

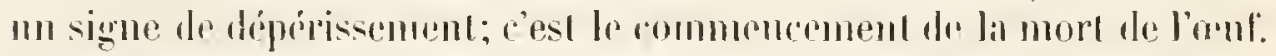
C'est dom: li un phémunime qu'on jeut dassor i juste titre an nombre de cenx anxpuels on assigne le nom de móntanmphose répressive. Nous

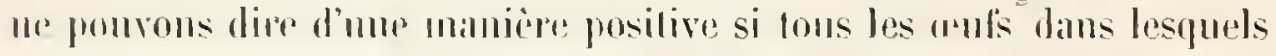

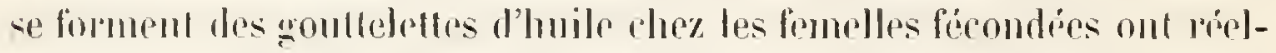

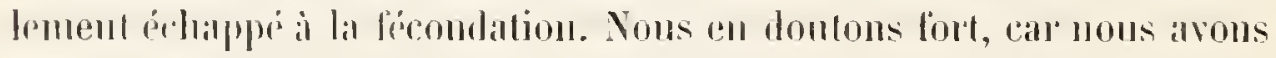

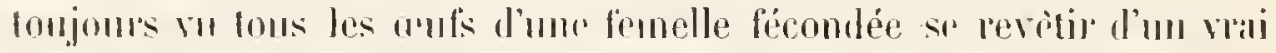

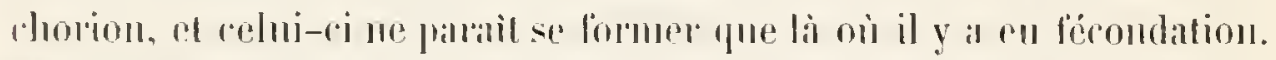

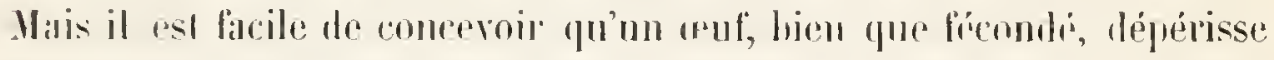

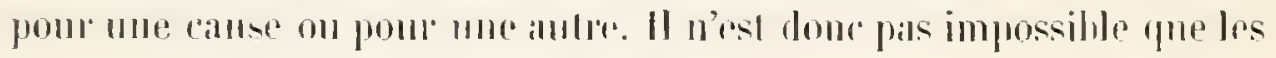

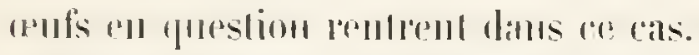

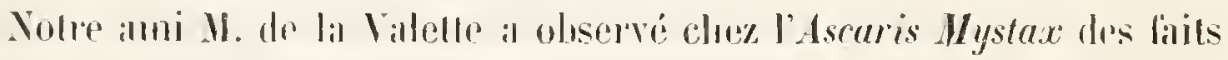

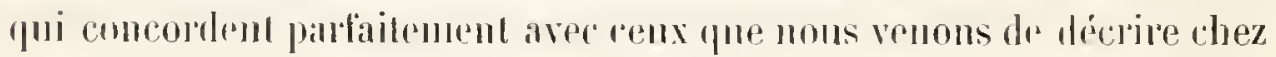
l'Ascaris suille. Il a en l'octasion d'observer une Ascaride du dhat nou

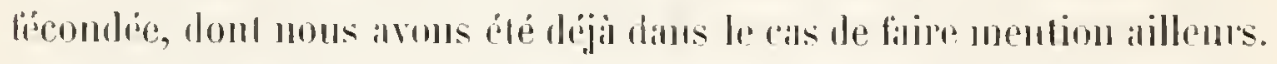
Une grambe pintir des aufs lrouvés dans lutérus renfermaient des goullalettes huilmsins all nombre fort varbable, frs mes grosses, les antres pretiles. Comme il nexistin pas un senl zoos prome dims les or-

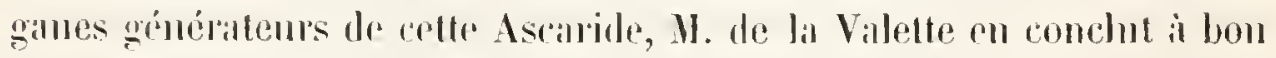
Iroit que la formation de res sonfles d'huile n'al absolument rien à faire avec les zoospermes. - De la Valctle chail arrive par snite de cette observation, toul i linil indipendamment de nos propres recher- 
ches, ì conceroir des doules sur l'exaclibule des lommées de Mrissner, relativement ì la mélamorphose grabseuse des zoospermes.

Chez une fenelle mon ficondere d'Oxymris vermicularis, nous avous tromvi la farlir des mélus qui aroisine le vagin si bien remplie de gonthelelles huileuses fort rélingentes, fur lorgane ou sous le micro-

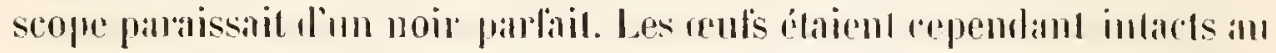
milien de cefle acemmulalion de gombeledtes. Noms nowons rim pu conjecturer sur la provenance de ces deprieres.

Nous ne voulons pas nier que les zoospormes an-mênes ne soienl susceptibles de subir me mélammphose regression. On lrome di el li

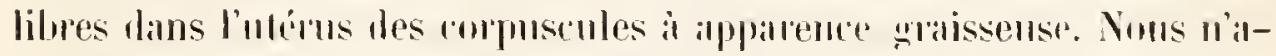

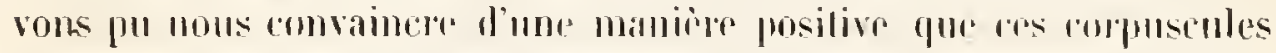

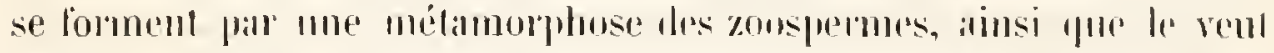
Meissner, mais la chose nest pas improbable. Il sagil soulement de

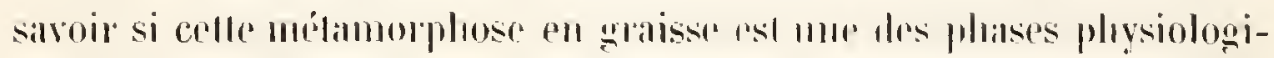

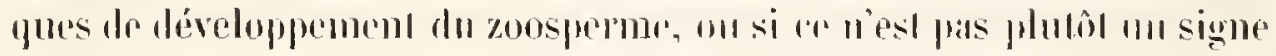

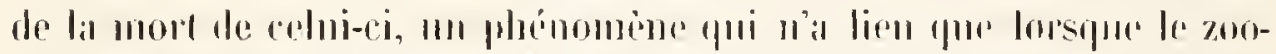

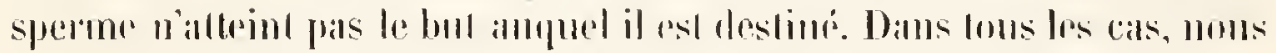

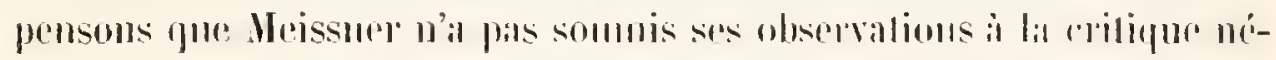
cessaime, puisquil n'a pas recomm la lombalim des gombes d'hnile

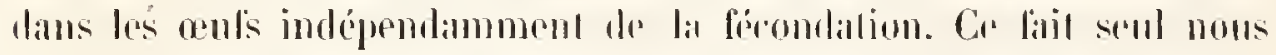

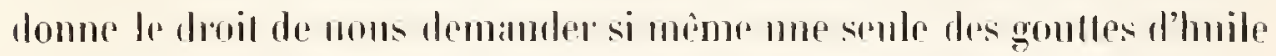

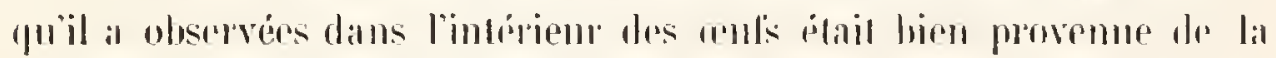

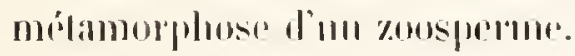

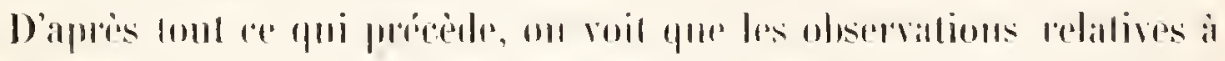

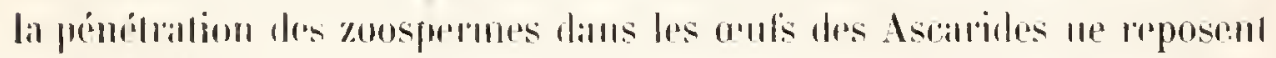

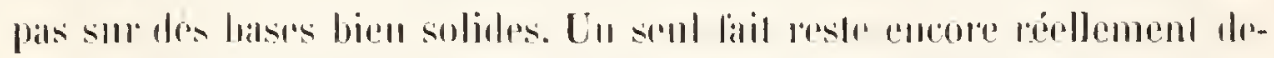
boul: Mrissury dil anoil valles zouspermes incomlestables dans linté-

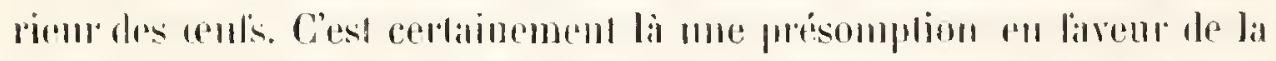

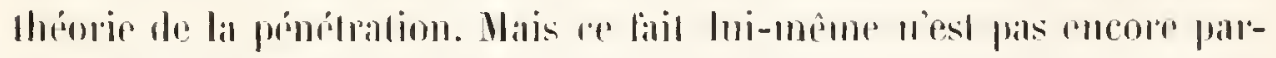

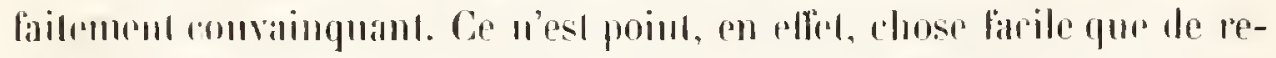

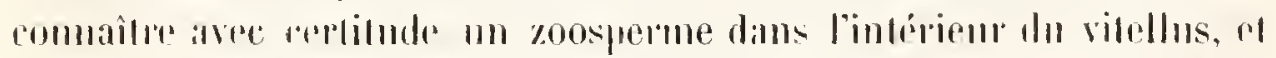

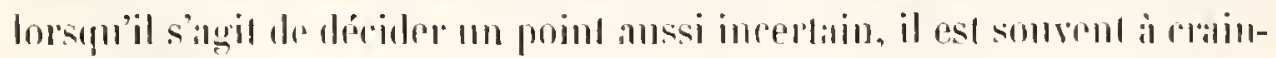


due que l'ail de la héorie ne soil plus prompe que celui de la saine

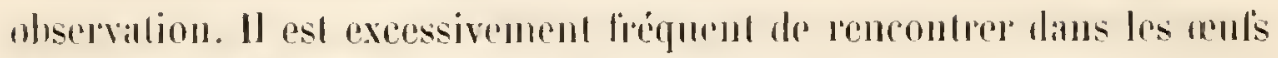

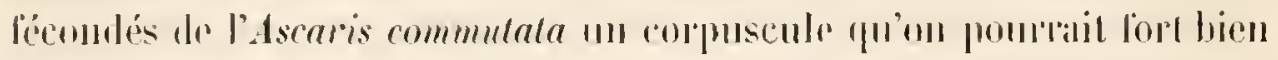

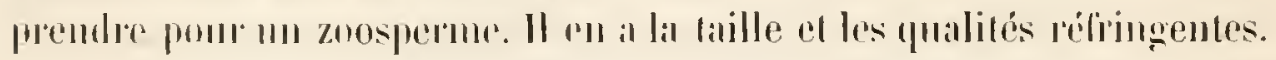

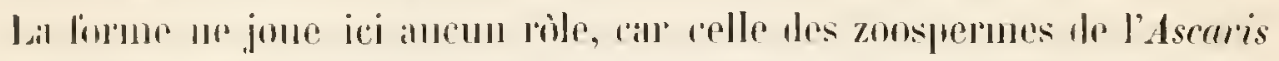

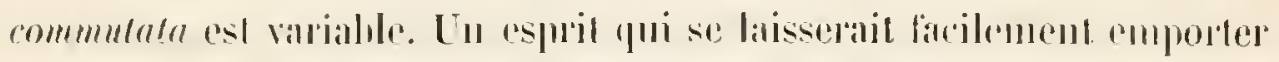

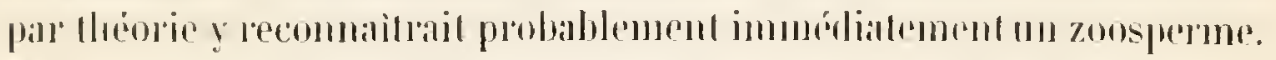
Sous ne pourons lien dire de posilif sur l'origine de ce corpuscule; ce-

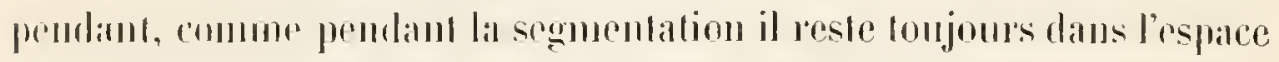
yui sípille les sphines de segmentalion du rhorion, il ne motes parait pas improhable quil doive atre compané a la résicule directrice de $\mathrm{H}$.

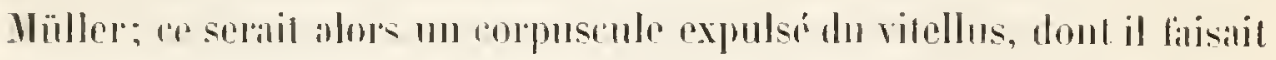
primitivement pantie. - Somme lomle, noms ne vonlons pas combester

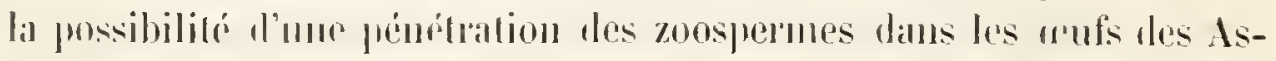

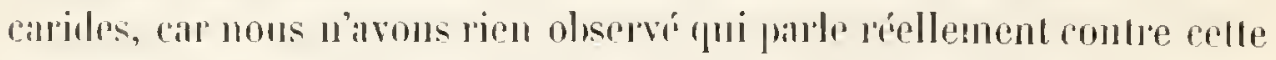

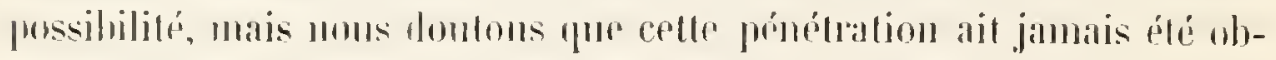
servó jusqüici.

Yons ne ronlons nons oceuper qur d'une maniere concise de la formation des rmberons, allendu que ce point me rentre qu'alecessoire-

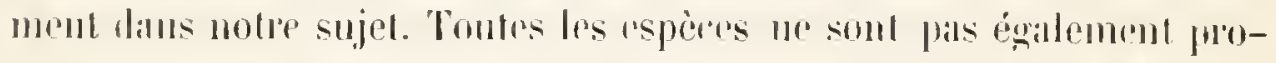
pres il fremellue celte élude. Puelques-mues, lelles pue l'Ascaris Mystax,

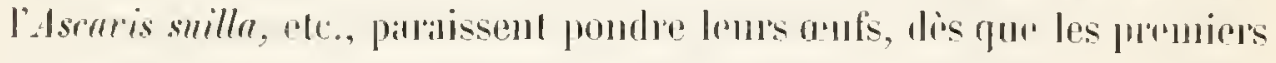
symptomes dr l'organisalion du vilellus se manifestent. D'antres, tulles yue l'Oxyuris rermirularis, l'Oxyuris sprirothera, l'Ascuris nigrorenosu,

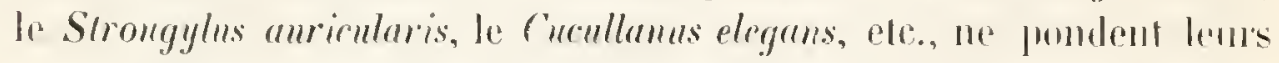

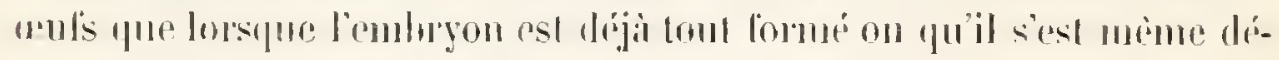

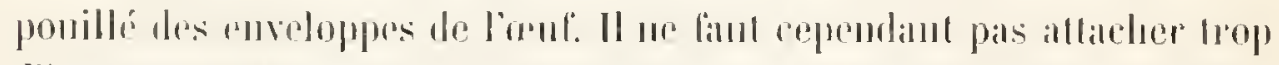

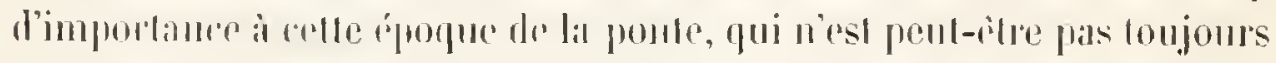

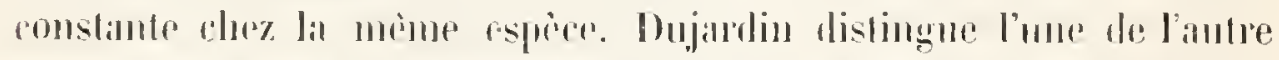

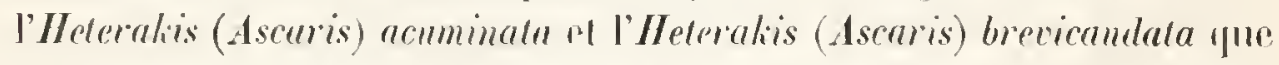
Diesing rémit sous la 11011 d'.1scaris acmminala. L'Heleralis acmminata "sl vivipare, selon Dujandin, Lamdis que l'Melerakis brevicandula est

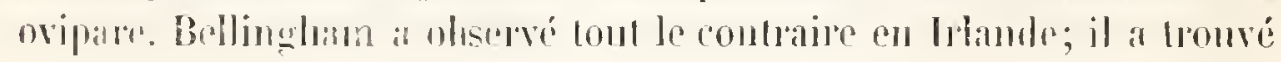


la première ovipare el la secoude vivipare. Nous avons loujours Irouvé

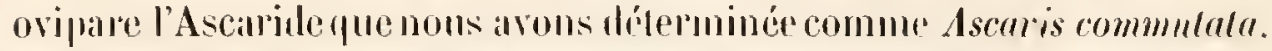
Un jour, cependant, mons avions placé mor vinglaine d'Ascarides ap-

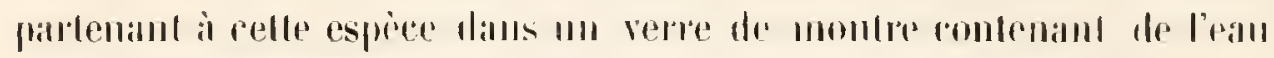

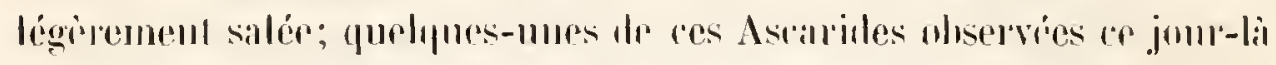

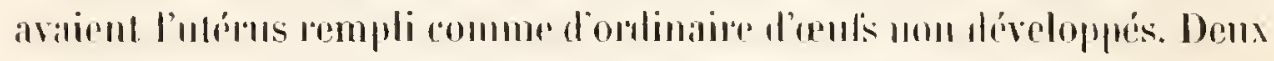

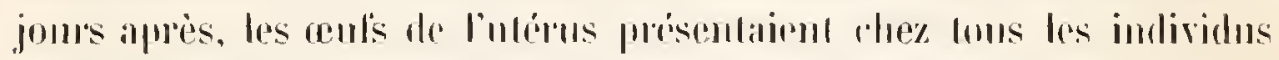
lous lis slantes de la segmentalion. Trois joms plus land les embryons

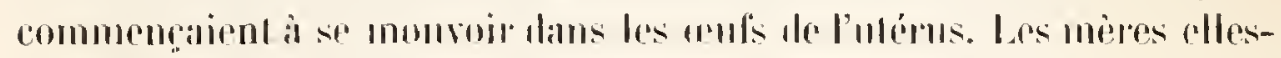

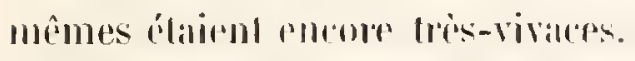

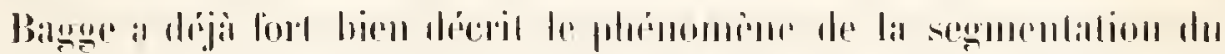

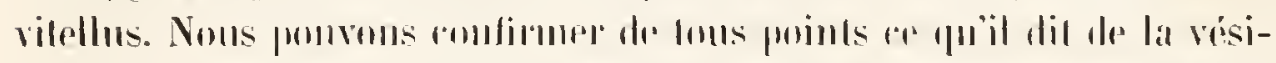

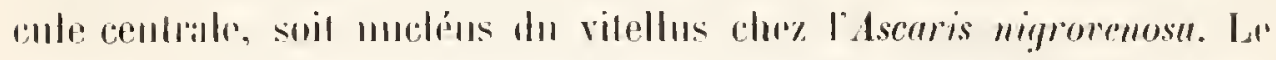

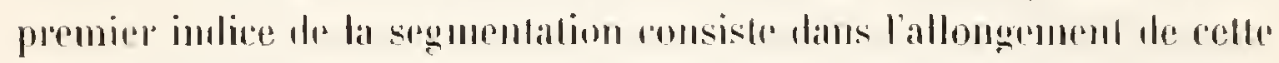

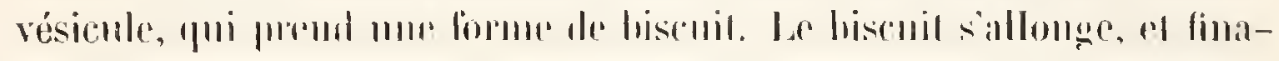

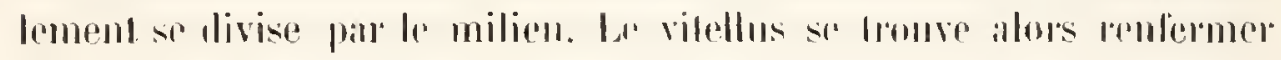

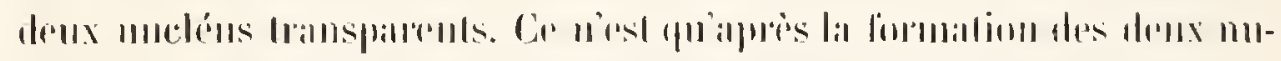

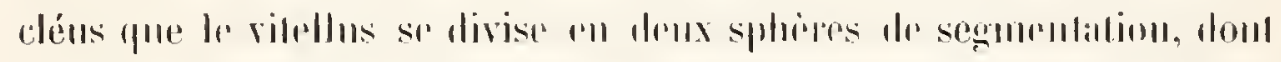

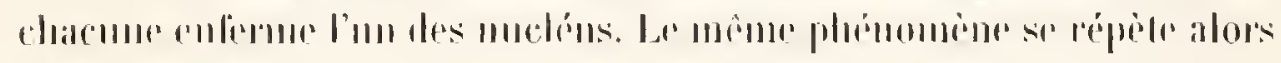

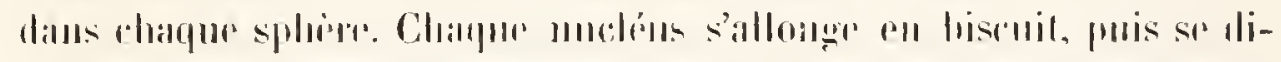

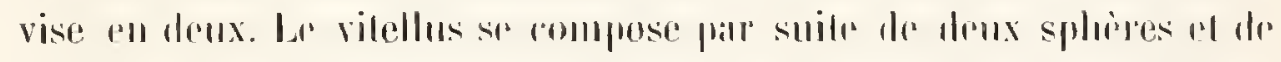

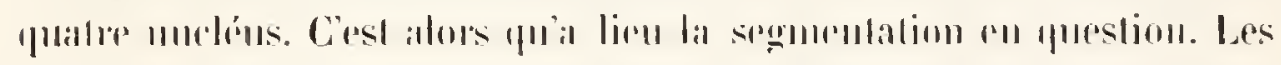

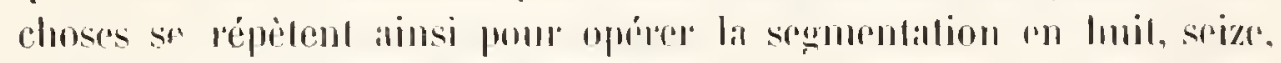

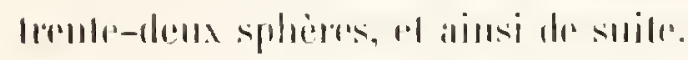

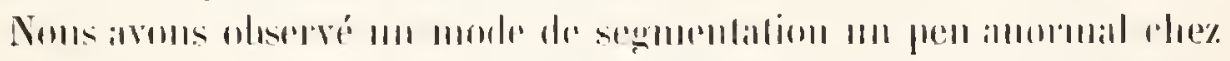

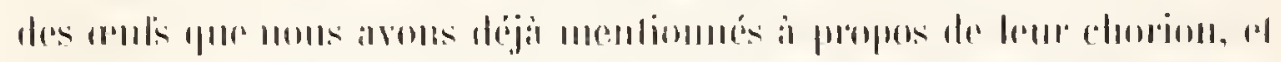

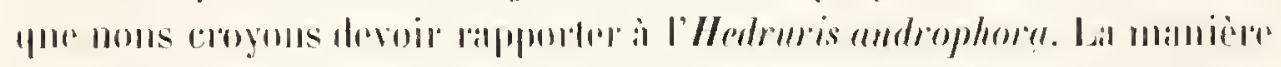

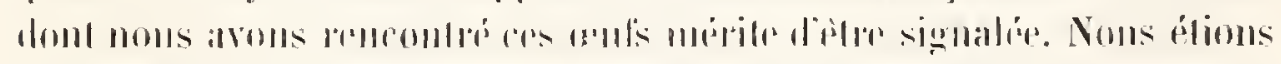

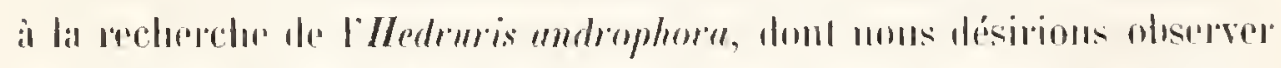

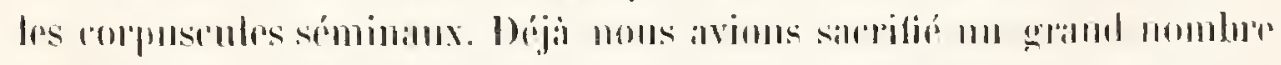

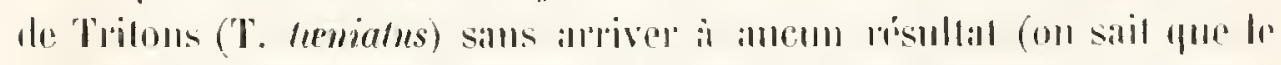

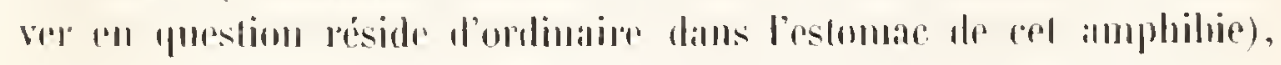

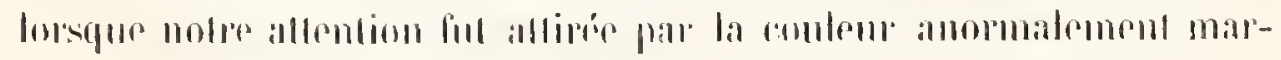




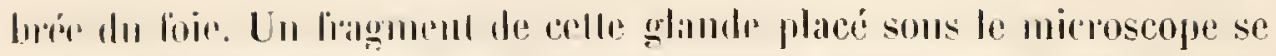

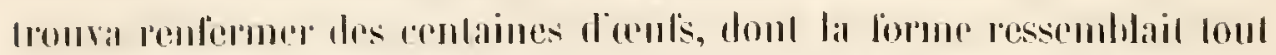

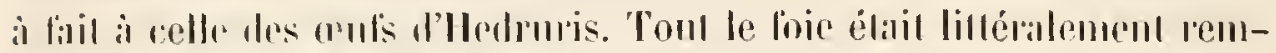

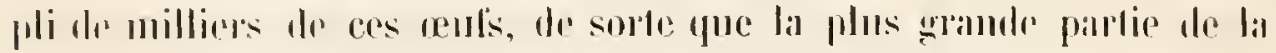

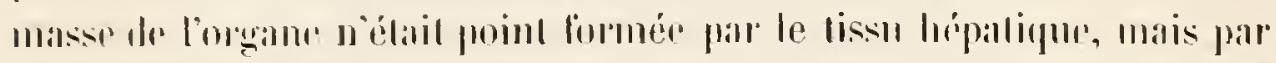

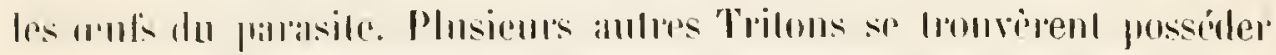

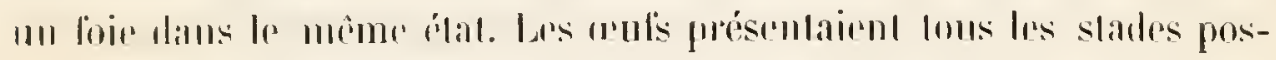

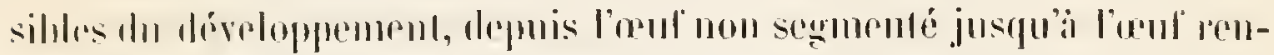
lermanl

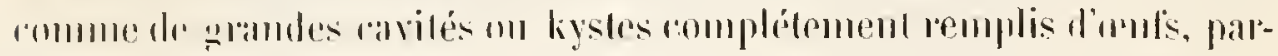

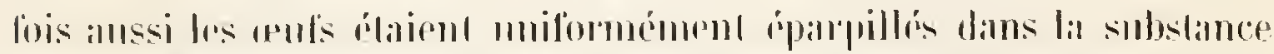

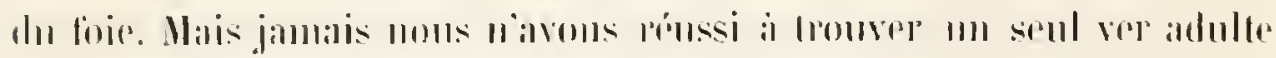
ni dins liostomale, ni dans linlestin, ni dins lo loie, ni dans antem an-

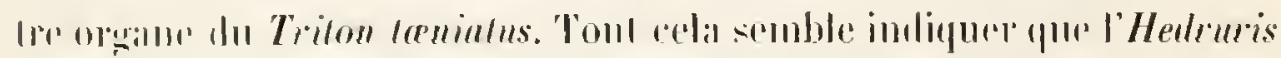
androphora

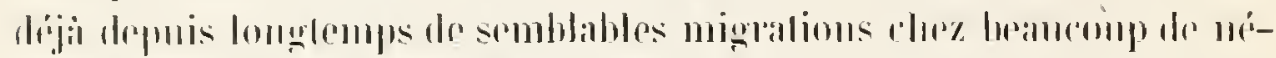

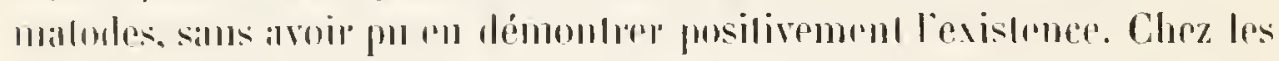

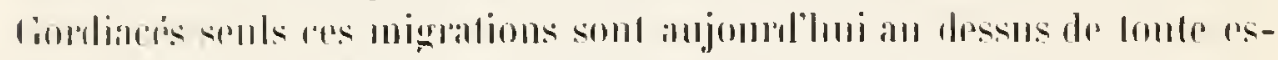

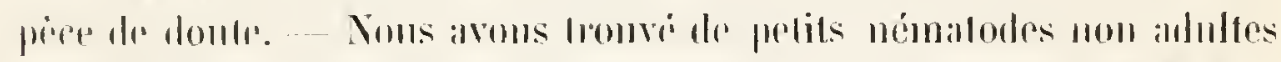

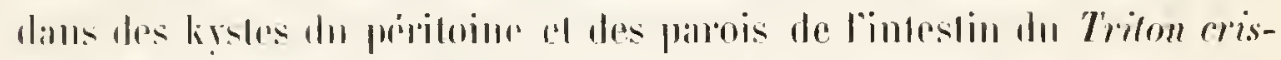

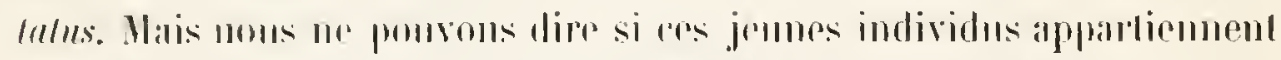

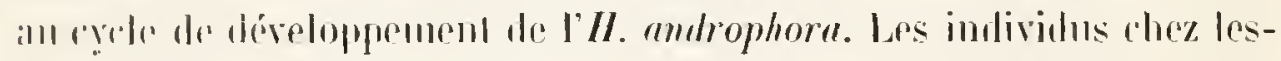

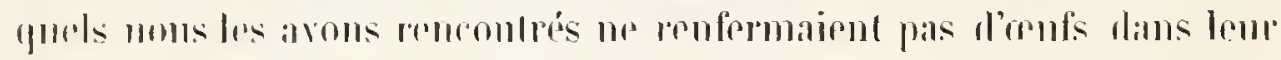
foir.

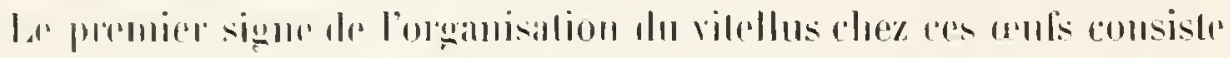

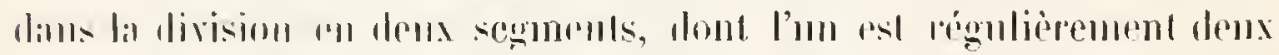

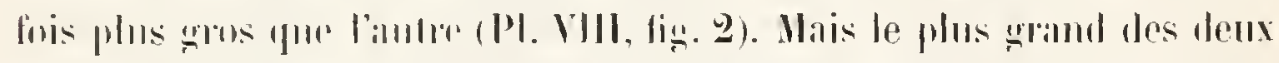

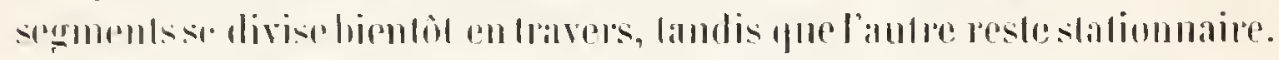

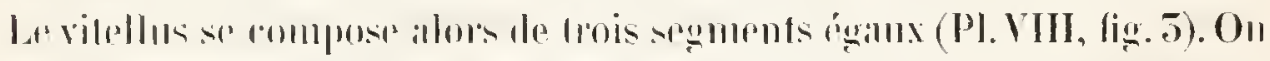

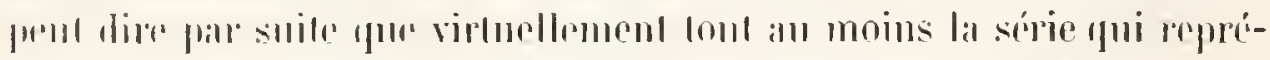

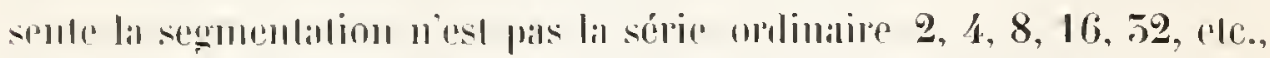

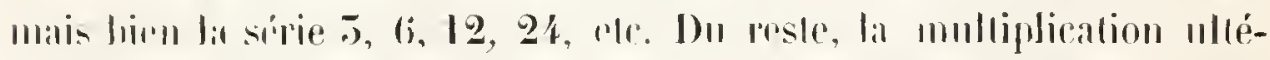

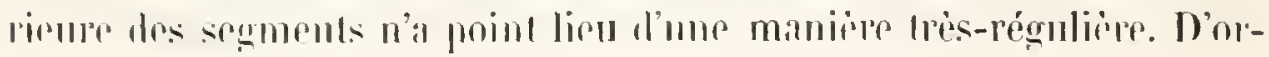


dinaine, l'mu des trois segments se divise phos promptrment pur les

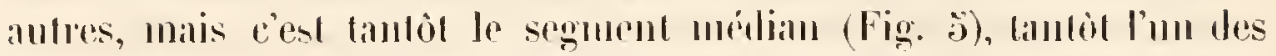
segments frominaux (Fig. 4), puis l'mu des demx autres segments suil son exemple (Fir. 6), el le troisiome linif de mime (Fig. T). Nous nalvous fas ponssé an delì nos observalioms sur le mode de division. L'enbryon, me fois tout formé, as relativement long a partanl pho-

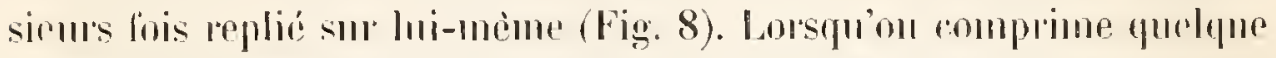
pen l'aul qui le renlermo, il sort par l'mu dre pöles. Nons anous vin, en

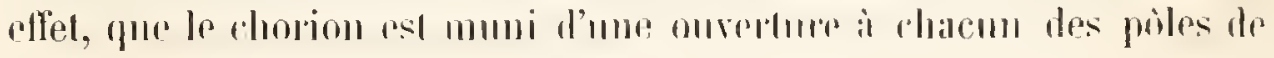
l'rinl'.

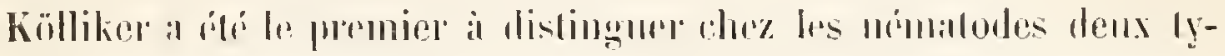

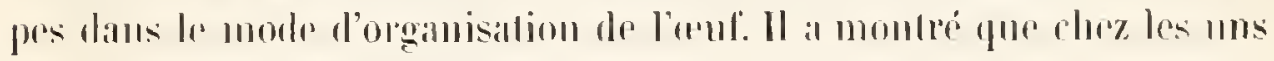

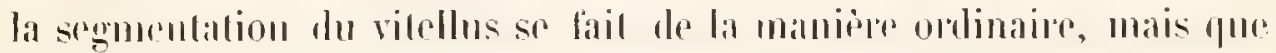

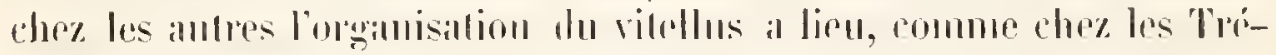

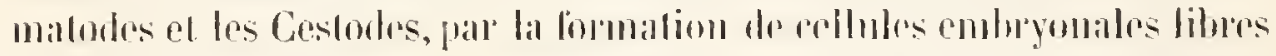

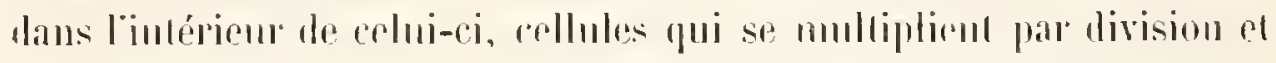

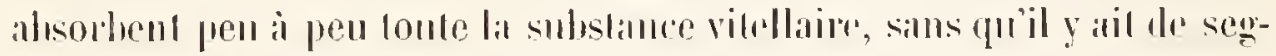

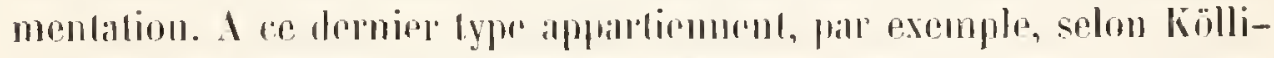
lin', l'asernis dentala ol l'Oxyuris mubiqum.

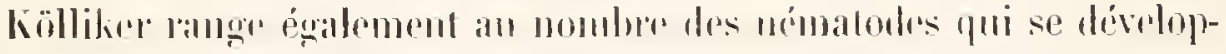
pont sans sogmontalion do vilellus lo Curnllamus oleyans. Ceest me

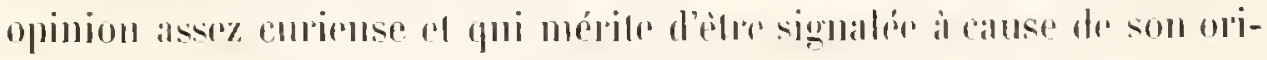

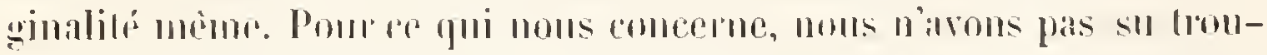

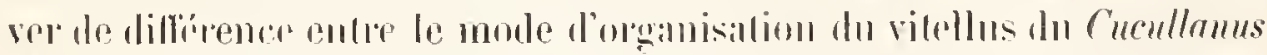

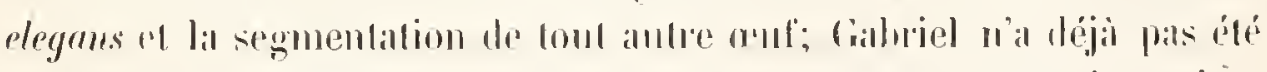

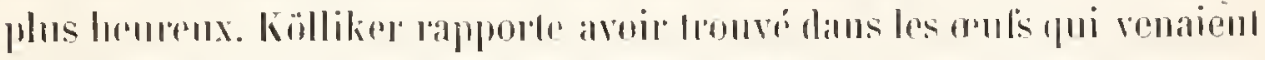

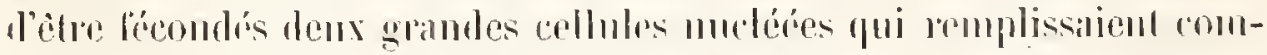

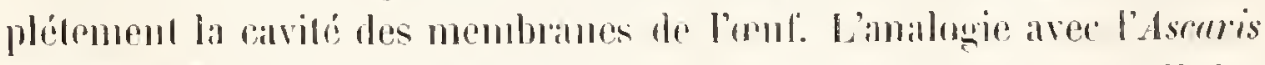
dentata le lorçail, dit-il, i considérer as cellules comme des cellules embyonalres; senlement dans l'un des as, celui du Curullamus, la première gónération des cellules ambryouales promplissail complétement la cavití de l'oul', dans l'antro celui de l'Ass. dentata, celte première grénéption était perdue an milien de la substamee du vitollus. 
Las lails observes par liöliker sont parlinitement exacts, mais les

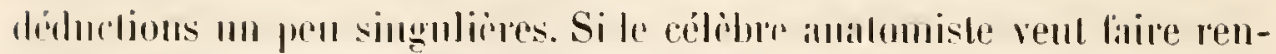

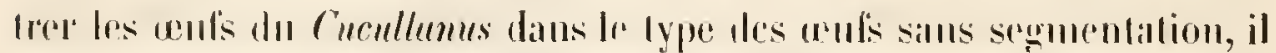

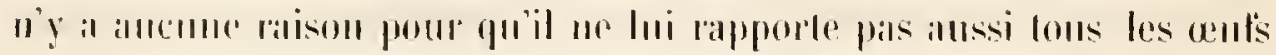

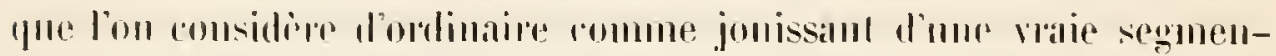

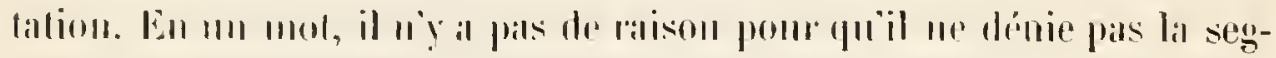

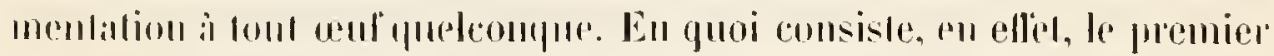

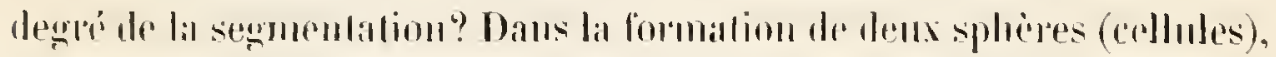

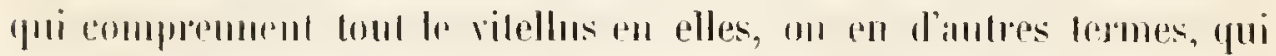

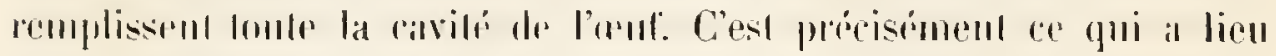

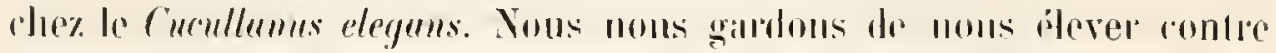

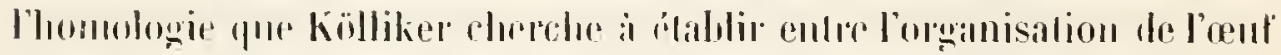

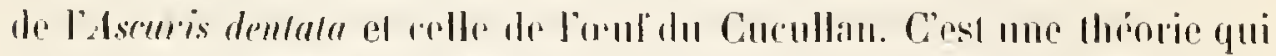

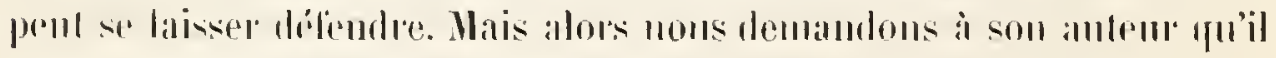

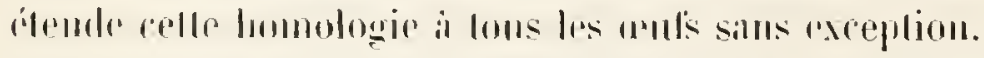

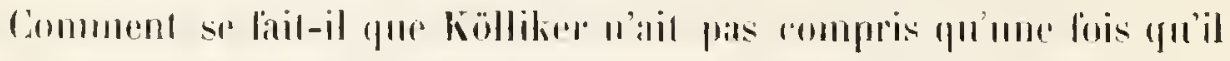

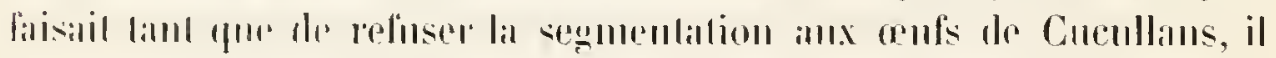

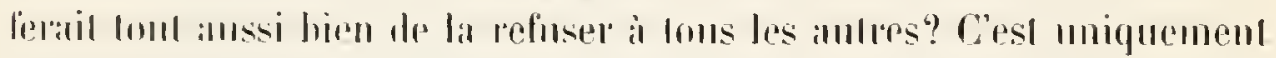

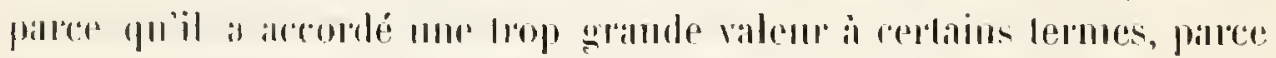

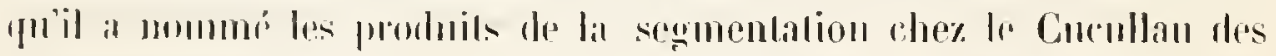

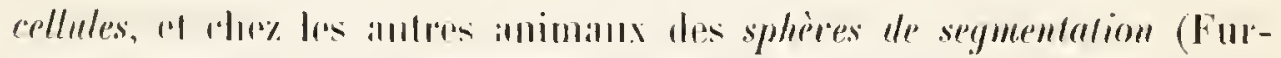

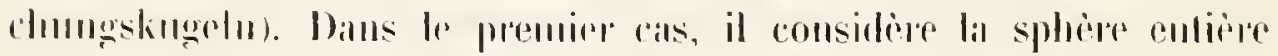

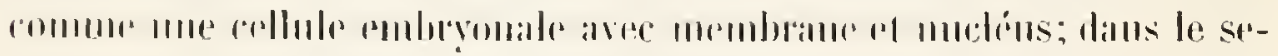

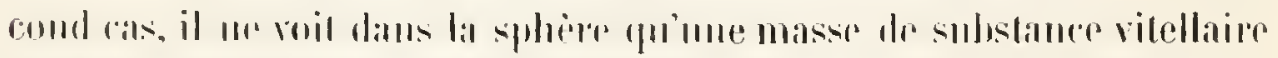

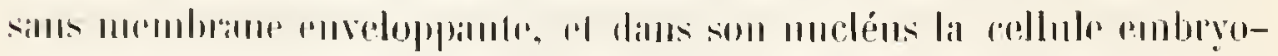

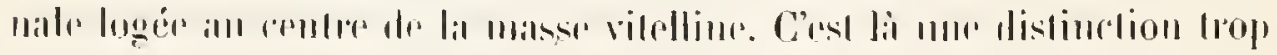

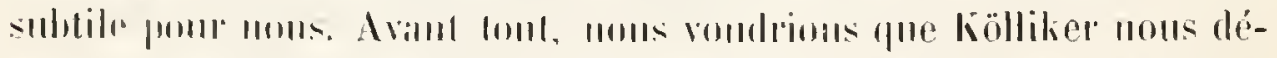

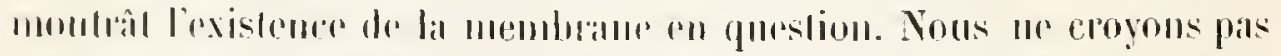

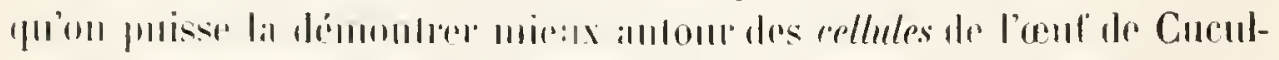

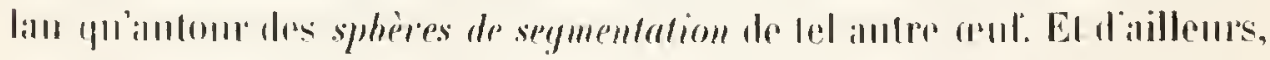

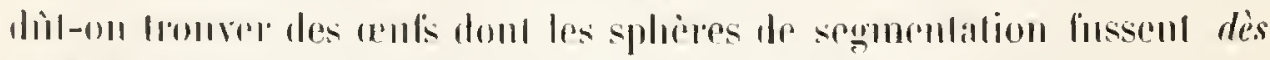

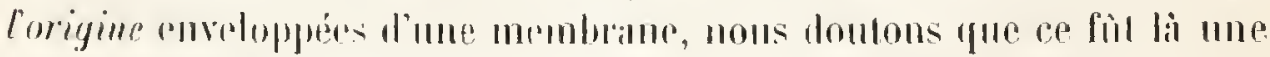

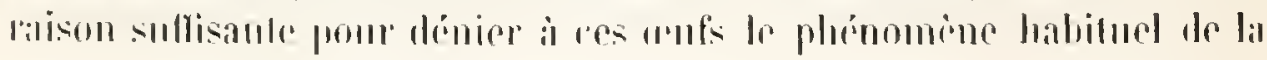


segmentation. Ricichert ne délend-il pas cncore anjond'hui l'existence d'une membrame amlour de ces sphimes dams tous les aul's?

Gabriel a déjà décrit la formalion de l'embryou doez le Cucullanus elegars. Mais sa descriplion est à pen près incompróhensible pall suite de ce qu’il a lion el un vilellus de mulrilion. Que colle distinclion de deux panties

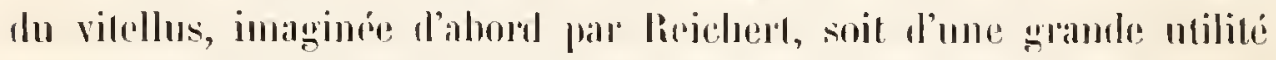
dans certains cas, e'est nne chose incontestable, mais il ne liml pas l'introduire là on elle n’a qure larire. Cetle dislindion est d'aulanl moins compréhensible, que Gabrich arlmol mue segnentalion lohale du vilellus. Or, on sail qur dans les gas où un vilellıs dr molrilion exisle, la seggmentation complible se restreint toujoms all vitellus de formation. Le vitellus se lramsforme dans son rnfier dans lembryou. Aussi la plualse dans larpuclle Gabriel nous dil que le vilellus git an dessus de l'embryou (super embryone jacet), ast-elle complétement inintelligible pour nous.

Tinndis que l'uuf du Cucullan se drveloppe, il ne cesse pas dre aroìtre dans toules les dimensions, sans doule par suile de l’absorplion du liquide qui le haigne. Lorsque la sogmentalion est sulfisamment avancée, le vilellus on plubil le corps de l'embryon prend la forme d'un cylindre ligèrement rombé, on si l'on ame mieus, me forme assez analogue à celle d'um concumlure (Pl. IV, lig. 7). A ce moment-li, il se compose de cellules bien distinctes, munies chateme d'un mucléus.

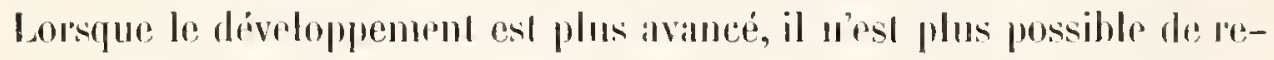
commaitre ce nucléus dans les cellules. L'embryon grandil faur degrés

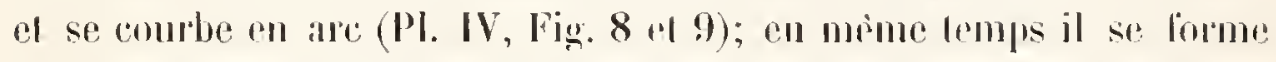

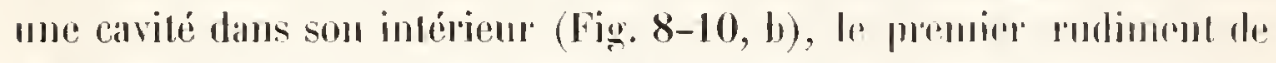

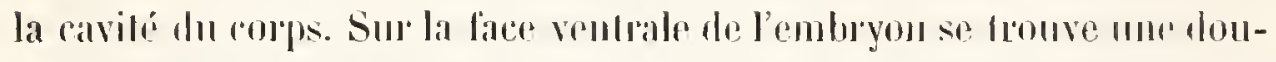
ble rangée longitudinale de cellules rui font une saillie en crite dims celle cavilo (Fig. 8-10, a). Ce soul ces cellules qui plus lard s'organisent el lorment le camal alimentaire. La suile du développenent noffie pas de particularité bien inferressinte; le ver croil en longuen el se replie par suite diversement sur hui-mème. Kölliker a rnis l'opinion que l'embryon dn Cucullan se forme par une division en spirate du 
vitellus, de sorte que l'embryon aurait dès l'origine la longueur maximum qüil atteint dans l'eul. C'est là une erreur manifeste qui ne résiste pas à l'observation. Liembryon croit en longueur comme tout embryon de nématode. Le seul fait que les embryons sont repliés de la manière la plus diverse daus lintérieur de l'ouf anrait dû faire concevoir à liölliker des doutes sur son hypothèse.

Kölliker dénie aux embryons qui riemnent de sorlir de l'ouf nonseulement l'anus et l'ouverture sexuelle, mais encore la bouche. Nous n’avons pas non plus pu reconnaître les deux premières de ces onverlures, mais la troisième existe bien réellement. L'absence d'ouverture génitale et d'anus semble assez générale chez les nématodes nowveaunés; le canal intestinal finit en pointe dans la partie postérieure du corps. Chez tous les embryous du Cucullan nous avons trouvé les hoyaux à cellules hien formés. Ce sont les organes que Gabriel désigne par glandula a me detecta chez les adultes, mais qu’il n’a pas vus chez les embryons. - Une autre particularité de ces embryons est de se servir de leur quene excessivement pointue comme d'organe fixateur. Lorsqu'ou observe sous le microseope un certain nombre d'embryons fraichement éclos, on en voit bientôt plusiemrs se fixer au porte-oljet ì l'aide de leur queue et exéuter les mouvements les plus divers avec le reste du corps. Mais le plus grand nombre se fixent les uns aux antres quene i queue, si bien qu'au bout d'une demi-lieure on ne manque jamais de trowver des rosettes de dix à vingt individus, rappelant tout a fait par leur distribution les yroupes d'œufs délachés du rachis de l'Ascaris suilla. Il est probable que cette propriété provient uniquement le l'existence d'une gouttelette d'une substance agglutinante placée ì l'extrémité de la queue. 


\section{DES MOUVEMENTS DES ZOOSPERMES.}

Jusquici les observations de Schneider sur les mourements des zoospermes chez les nématodes n’ont trouvé ni contradicteur ni léfenseur A priori, il n'esl guère permis de douter de l'cxaclitude de la description de Schmeider, laynelle dénote un soin tout à fait scrupulenx dans la méthode "l la poursuite des recherches. Mais il s'agit de saroir d'une part si les corpuscules avee lesquels il a en à laire sont bien de vrais zoospermes, ou si ce ne sont peut-être pas des êtres élrangers, des parasites, diulre part si les mouroments observés sont bien réellement IIn phrinomene physiologique.

Duraut ces dernières années on s'est habitué toujours plus à voir les éléments les plus simples de la nature organique présenter des phénomènes de contractilité, rippelant plus on moins le mode de progression des Anocbas. Sans vouloir parler ici des corps amobiformes qui, d’après les observations de Lieberkïhn, sortent des pseudonavicelles el qui apparticment an cycle de développement des grégarines, nous pourons citer loule une série de corps dont la nature unicellulaire n'est pas suspectée el qui présentent une contractilité de ce gemre. Tels sont, par exemple, les corpuscules incolores du sang des vertébrés, les corpuscules muqueux des mollusques, les corpuscules sanguins de divers invertébrés, de l'écrevisse (Astarns fuciatilis), par exemple. Tout récemment encore Lenckint a déril des mouvements amobiformes chez les cellules hépatiques du lapin, et Kölliker clıez les cellules dı mantean des Ascidies, ef les cellules du tissu cenjonctif grélatineux de la lorpille électrique (Torpedo ocellata). Les mouremenls observés par Busch chez les cellules pigmentaires d'une larve de grenonille doivent aussi sans donte rentrer dans cette catégorie. Les observations de Schneider ne seraient donc qu'un anneau de plus à celte chaîne de phénomènes. 
Sclmeider n’a pas été le premier à comparer les corpusenles séminamx des némalodes avec des Amohals. C'est Bischoff qui, dans sa première réfulation de Nelson, s'est d'abord servi de cette comparaison, mais d'une manière toute différente que Schneider. Les corpuscules séminaux (il prarle de ceux qui sont pris clıez le mâle), dit-il, placés dans leau pure, commencent á développer des expansions en forme de gouttelettes on the prolongement, yui sont formés par du sarcode. C’est lìu elfet de lömbibition. "Cescorpuseules, ornés de leurs expansions, ressmblent somvent, ì s'y méprentre, à un corpuscule sanguin d'un écrevisse ou ì un Amobia; parfois mème, lorsquion observe un corpuscule dont les expansions sont en voie de se former, on eroirait voir un Amoba pendant la progression. " - Comme on le voit, Bisrhofl n’a cru avoir et n'a probablement en sous les yeux yu’u phénomène anormal produit parr la diffusion.

Selon les prescriptions de Sclmeider, nous avons ouvert tous les nématodes ì observer daus de l'albumine on bien dans une dissolution saline ou sucrée. Le plus fréquemment nous arons en recours à une dissolution de sel marin. Pas me senle fois, quelle que fìt du l'este l'espèce qui faisail le sujel de l'observation, nous n'avons réussi ì roir des mouvements amorhiformes chrz des zoospermes pris dans la résicule semminale dn màle. Celte observation concorole parfatement avec ce qu'a vu Schneider. Mais le résultat fut tout antre diss que nous nous adressimes ì des zoospermes pris dans l'oviducte, la poche séminale, ou l'utirus de crltains nématodes femelles. Parmi les espèces que nous arons observes, il en est une qui est plus propre que toutes les autres à l'étude des mowrements des zoospermes. C'est le Strongylus auricularis. C'est donc dr hi que nous nous occuperous d'ahord.

On est fripprides le premier abord de l'existence de corpuscules trèsdivers dans les organes génératemrs des strongyles femelles. Bagge a déjà signalé le fait que la vésionle síminale du mâle renlerme des corpuscules tout ditfírents de cenx qu'on est tenti de considérer comme rais zoospermes chez les fentelles. L.es premiers sout ceux que nous arons déji décrits plus laut; les autres sont de petites résicules rondes 
munies d'un nucléus très-dislinet. Cette observation est en elle-mème parfaitement exacte, mais elle est inconplete. En effet, on trouve dans l'utérus et dans l'oviducte des femelles non seulement ces petites vésicules mucléées, mais encore des corpuscules pirfitilument identiques aux zoospermes de la vésicule sćminale llu màle, puis enfin d’autres corpuscules a forme plus ou moins irréguliere. En observant ces derniers aree quelque attention el quelque suilr, on HIr larde pas à en trouver un certain nombre qui exentent les monvements décrits par Sclmeiler. Ces mouvements sont e'u général asco\% lents, dr sorte qu'il

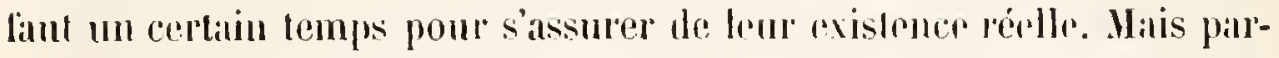
lois un corpuscule qui jusyualors avail áli lorl lenl dans ses mouvements, se mel tout à conp i exicuter unc serio de mourements asse\%

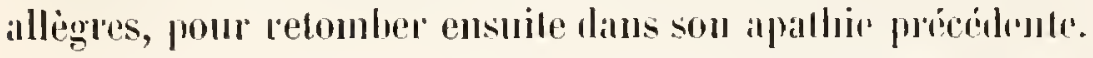

Il est facile de se convaincre qu'on n'il pas it linir lit ì do simples phénomenes de dillusion semblables it cenx détrils par Bischoff chez l'Ascaris Mystax. En effel, ces mourenents durinl dorz lo mòne corpuscule pendant phosieurs hem'es de suite, ils som mime d'ordinaire phs actifs all bout d'une heure quau commencentent de lohservation. - Il serail possible cependant, comme nous l'arous dejai indiqué, que ces corpuscules fussent des amimalcules parasites. Schneiler a dejjà paré ì cette objection, puisquil aflime que toutes les différentes formes de corpuscules quon tronve en outre des wuls dims lo lube générateur du Strongyle femelle, ne sont que différentes blapes lans le cycle de diveloppentent des zoospermes. Celle assertion est parficitement exacte. Nons allons en démontrer la vérité dans ses détails, puisque Schneider s'rst contenté de donner un résultat sommaire de ses observiltions.

On trouve d'iabord diuns le mbe générateur limelle des corpuscules (PI. I, fig. 22) dont la forme est identipuement la même que celle des zoosprimes qui ont alteint leur plus haut degré de développement dans les organes mâles. Ciest la forme que nous avons dẹja comparéc plus haut à une come de chamois. Ces corpuscules-lì sont dépourvus de toute espece de mobilité. Parfois on en tronve quelques-uns dont la 
base est comme traversée par un mème bâtonnet ou appliquée sur lui (Fig. 22 a). Ceux-ci ne sont pas plus susceptibles de se mouvoir que ceux-là. A côté de ces corpuscules sion trouvent d'autres dont la forme générale est parfaitement la même, arec cette différence que la corne, au lieu d'être nettement coupée à la base, s'élargit dans le bas en une partie transparente, peu réfringente ef diversement lobée (Figr. 25). II ne peut pas y avoir de tonte que ces corpuseules ne soient une simple modification des précédents, ef cependant ils sont drajia susceptibles de se momvoir. On roit les lobes da la parlie basilaire moditier leur forme, sallongrer et se rétracter. Cependant, aussi longlemps que le zoosperme conserve cette forme, les mouvements sont en général fort lents. Parlois senlement la base du zoosperme lait un monvement tel que la pointe de la corne soit obligée de décrire assez lorusquement un are de cercle consilérable. La suite de la métamorphose du zoosperme consiste en ce que la partic qui fait les mouvements devient toujom's plus consilérable, tandis que la pointe privée de momements propres diminue dans la même proportion. En un mol, la partie en forme de corne se fond peu i pen dans la partic amobiforme (Fig. 2.1). Sous cette lorme le zoospermo exćcute déja les mouvements plus rapides, mais des mouvements ressemblant souvent encore phus à ceux d'une Astasic qu’ì cem d’un Amoba. - Enfin, la pointe de la corme finit par disparaitre complétement. Le zoosperme a pris tout a lait l'apparence d'un Anoba. Son corps se contracte cependant de manièe à lormer me sorte de boule qui n’émet de prolongements en expansions que d'un seul cotté (Figg. 23). C'est alor's que les mourements atteignent leur maximum d’intensité. Le plus souvent les expansions sont larges, courtes et aplaties. Parfois anssi elles sont Iris-minces, longues et diversement ramifiées. Ce u’est guère que dans ce dernier cas qu’on peut parter d'une certaine vivacité dans le développement ef la rétraction des expansions. Nous avons représenté -dans les figures 200,26 et 27 une série de formes présentées successivement par trois zoospermes dans l'espace de deux à trois minutes.

Une grande partie des corpuseules de la dernire forme décrite sont 
munis d'un nucléus allongé, ce qui leur donne une grande analogrie le forme avec les cellules nucléées que Bagge a été le premier à décrire dans les organes génilaux l'emelles du Strongyhus auricularis. Nous ne doutons pas que ces cellules (Fig. 29) ne soient le lernier terme du cycle de développenent des zoospermes, mais nous ne samrions dire d'une manière positive si ces cellules mème sont encore susceptibles de faire les mourements amobiformes. Nous avons bicn vu des corpuscules (Fig. 28) faire ces mouvements, alors qu'ils n'émettaient qu'une. ou deux expansions des plus minimes d'un point délerminé de leur pourtour, el que du reste ils étaient parfailement identiques aux cellules à nucléus allongé, mais nous n'avons pas vu de cellules primitivement dépourvues de toute expansion, commencer subitement à en émettre. On peut du reste fort bien suivre la formation des nucléus allongés. On trouve des zoospermes qui exécutent des mouvements amobiformes, toul en étant munis d'un petit nucléus rond. Chez d'autres ce nucléus est déjì un pen allongé; chez d’autres enfin il a pris entièrement la forme du nucléus des cellules de Bagge. Ces deruières ont un diamètre de $0^{\mathrm{mm}}, 006$. Sous l'action de l'iode, le nucléus se colore d'une teinte beaucoup plus foncée que la membrane el le contemu de la cellule.

Il est du reste nécessaire d'étudier un assez grand nombre d’individus pour s'assurer parfaitement du passage de chacune de ces formes à la suivante. Souvent toules les formes ne sont pas reprósentées. Il n'est pas rare, par exemple, de l'ouver des femelles dont les mrames générateurs ne renferment que des zoospermes appartenanl aux formes que nous avons représentées dans les figures 22 et $\mathbf{2 5}$. Toutes les formes suivantes manquent. Ce firit s'explique sims doute par la circonstance que ces lemelles ont élé fécondées depuis fort peu de temps.

Chez l'Ascaris commutata nous avons ohserve des laits toul semblables. Les zoospermes sont senlement ici en général très-délicats. Lor'sque la solution saline est trop pen concentríe, les zoospermes périssent rapidement par suite d'actions de diffusion. Lorsqu'elle est trop concentrée ils se contractent et périssent également. Il faut lonc garler un 
juste milien, qui n'est pas toujours facile à trouver. La même chose a lieu du reste pour les zoospermes du Strongylus auricularis, mais la lalitude dans le degré de concentration de la solution paraît ètre plus gr'ande. Chez celte Ascaride, comme chez le Strongylus auricularis, il se forme un nucléus dans l'intérieur des zoospermes qui font les mouvements amobiformes. Mais cette fois le mucléus est gros et arrondi (Pl. VII, lig. 6).

Nous arons également confirmé les observations de Schmeider chez le Cucullanus elegans. Ce ver est tont à fait impropre à des observations le ce genre. Les corpuscules séminanx (PI. IV, fig. 15) qui remplissent les poches séminales de la femelle sont si petits $\left(0^{\mathrm{mm}}, 003\right.$ à $\left.0^{\mathrm{mm}}, 004\right)$, qu'ils sont en proie à un tremblement moléculaire continuel. Comme ils sont en général munis d'expansions irrégulières toutes placées du mème côté, il arrive soment ru'on croit voir ces expansions s'allonger el se racentrcir, lambis que ce n'est lì qu'une suite de tremblements, qu'une appaltente produite par un chamgentent de position du zoosperunc. Ceprendant, apres lien des peines nous sommes arrivé à nous convanincre que cliez le Cucullanus anssi les mouvements des zoospermes ont lien. - Une senle fois nous avons en l'occasion l'observer ces monvements l'un' manière splendide. Dejja, à la simple observation an fravers des parois du corps de ce ver, on s'apercevait que les poches séminales ot les oriductes étaient remplis de corpuscules d'une forme toule particulicre. Les organes générateurs ayant été isolés el ouverts, ces corpuscules prósentèrent la forme suivante. C'étaient des bàtonnets longs ef minces, munis le plus soment dans loute leur longueur de dentelures al de courtes branches (PI. IV, lig. 14). Beancoup d'entre "ux étaient bifurquís (Fig. 14, a), d'autres diversement ramifiés. Ils alteignaient jusqu’’a me longueur de $0^{\mathrm{mm}}, 05$. Ces bâtonnets prósentaient des monvenents amobéens de la maniere la phus exquise; les uns restaicut parfaitement immohiles, mais les antres retiraient leurs petites branches, cu laisaient croître d'autres, s’élargissaient ici, s’amincissinimt li, et ainsi de snite. Il serait possible que ces corpuscules fussent des parasiles, el nous n'oserions pas le nier d'une manière po- 
silive; cependant, ce que nous savons des zoospermes d'autres nématodes, nous permet de supposer avee une assez grande vraisemblance que les dits corpuscules étaient aussi de véritables zoospermes. Il subsiste, il est vrai, cette circonstance peu explicable que, parmi une quarantaine de fiemelles observées, une seule ait renfermé des zoospermes à cedegré de développement. Cette forme n'est du reste point la seule qui soit apte à opérer la fécondation, car les ceufs se développent parfaitement bien chez les femelles qui ne senferment que des zoospermes représentés dans la figure $\mathbf{1 5}$.

C'est en vilin que nous avons essayé de constater l'existence de mouvements amobiformes chez les zoospermes de l'Ascaris Mystax et de l'Ascaris suilla. Si ces zoospermes sont également doués de mobilité, comme leur base irrégulièrement lobée el floconneuse pourrait le faire supposer, leurs mouvements sont certainement excessivement lents. Schmeider dit dejä avoir vainement cherché des mouvements rephateurs clez les zoospermes de l'Ascaris suilla.

Nous n'avons pas en l'occasion d'observer l'Angiostoma limacis, chez lequel Sclmeider a découvert d'abord la reptation des zoospermes. Mais nous avons appris dernièrement de Guido Wagener et $\mathrm{N}$. Lieberkühn qu’ils avaient répété les observations de Schneider sur ce ver et qu'ils les avaient trouvées prarfaitement exactes. Ces observateurs doutent seulement que l'animal coïncide avec l'Ang. limaois de Dujardin.

L'existence des mouvements amobéens chez les zoospermes des nématodes ne peut plus être révoquée en donte, bien que nous ne soyons pas en élat d’adınettre la généralité du phénomène. C’est un fail qui rendra plus facile à comprendre l'acte de la pénétration du zoosperme dans l'ouf, à supposer que cette pénétration doive être démontréc avec certitude un jour. 


\section{COUP DOEII, RETROSPECTIF.}

Il ne sera peut-ibe fias sans indéril pour le lectemr do pouvoir à la fin te ce lavail joler un comp d'uil rapide sm les principanx résultats acquis. Nons pensons done laire birn de clore ces pages par une suite

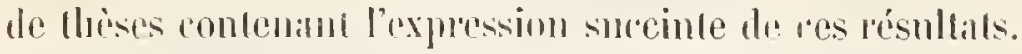

1. Les corpusenles que Bischoll a décharés, chez l'Asc. Mystux, n’ùtre que des romules épithélianx, sont de veribahles zoospermes, ainsi que Nolson el Meissner l'avaicul somlenu à juste litre.

11. Cest it fort qur, Veissner admet chez l'Ascaris Mystax m rachis apparent formé par me pile de cellules-gernes. Ces domières n'existent pas.

III. 'Le micropyle décril på Meissner chez les auls de l'Asc. Mystax. n'existe pas.

IV. Lélément primaire tles aufs chez tous les nématodes est la vésicule germinalive.

Y. L'opinion sutemur par Nelson el Meissner que la fŕcondalion des ouls d'Asparides est un résultal de la próntration des zoospermes dims

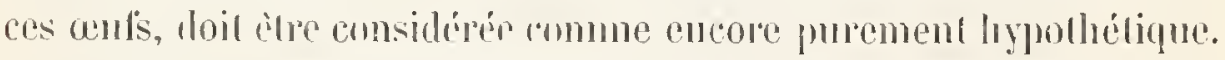

11. Les oufs of les zuospermes présentent dans leur dévoloprement respectil une homologie morphologigue parfaile.

VII. Les fails relatils anx mouvements des zoospermes qui ont été observés par Schneider chez divers nímatodes sont parfintement exacts. 


\section{EXPLICATION DES PLANCHES.}

\section{PLANCIE I.}

Fig. 1. Cellules épituéliales prises dans l'utérus de l'.1scaris suilla, et vues de face.

Fig. 2. Cellules épithéliales du même organe. vues tle profil.

Fig. 3. Cellules épithéliales de l'uvilucte de l'A scaris Mystax.

Fig. 4. Cellulos épithéliales de l'ulérus du Cucullanus eleguns, vues de face.

Fig. 6. Cellules épithéliales du mèue, vues de prolit.

Fig. 6. Portion de l'utérus du Cuinllanus eleguns, vile.

Fig 7. Portion de l’oviducte dı Cucullames elegans, pour montrer l'épithélium.

Fig. 8. Portion du vagin de l'Oxyuris rermicularis, dans les parois duquel on remarque les fibres museulaires lranstersales.

Portion du conduit éjaculateur de l'Ascaris mucrona ta; a cellules museulaires, $b$ épillélium.

\section{PLANCIIE II.}

Fig. 1. Organes copulateurs le l'Ascaris suilla mile; $a$ spueules; $b$ intumescence il la base des spieules; $c, d$ muscles rétracteurs des spicules; $e, e^{\prime}$ varicosités des tégunents; $f$ fente génito-anale.

Fig. 2. Partie supérienre d'un spicule; $a$ spicule; $b$ cellules placées i la base du spicule; $c$ muscles rétracteurs.

Fig. 3. L'une des eellules situées a la base lles spicules, traitíe par l'acide acétique.

Fig. 4-z̈. Varicosités des téguments, fortement grossis.

Fig. 6. Partie postérieure du Cucullanus elegans mâle; $a$ tube alimentaire; $b$ conduit “jaculateur; c raricosilés des líguments : l grand spicule; e petit spicule; $f$ anus.

\section{PLANCIIE III.}

N. B. Toutes les figures de cette planche concernent l'Ascaris suilla.

Fig. 1. Partie de la rérion supérieure du vitellogìne; $b$ vésicules germinatives.

Fig. 2. Parlie de la régrion moyenne du vilellogène; a paroi du vilellogéne; $b$ indication des côtes longrtudinales; $c$ rudiments des auf3; $d$ rachis. 
Fig. 3. Trois aufs munis de leur vésieule germinatis et pris dans la région moyenne de lovaire.

Fig. 4. Fragment du eglimde formé par les unfs argomérés daus le vitellog̉ene. La plus grande partie des arufs ont été arrachés avee des aiguilles afin de mettre le raelis à découvert.

Fig. 3. Groupes d'enfs en étoile prouhit par la dilacération artilicielle du rachis.

Fig. 6. Euf pris dans l'oviducte.

Fig. 7-12. Expulsion riraluelle d'un fragment du vitellus.

Fig. 13. Fragments de vitellus expulsís des cenfs.

Fin. 14-13̈. Osufs pris dats l'utérus d'une femelle non lécondée; " membrane vitelline; $b$ chorion.

Fig. 16-18. Gufs pris dans l'mtérus d'une femelle non fécondée et contenant des gounteltes d'huile dans lrur intérieur.

Fing 1S-20. Eufs pris dans l'utérus d'une femelle fécondée; a membrane vitelline; b ehorion.

\section{PLANCHE IV}

X. B. Les ligures 1-1't concernent le Cucullanus elegans.

Fig. 1. Sommet de l'ovaire contenant les orules.

Finn. 2. Partie de la rigion médiane de l'ovaire, contenant des œufs plus développés.

Figr. 3. Partie de la colonne d'ceufs contenue dans l'ovaire, isolée.

Fig. 4. Deus auls de la partie moyenne de l'ovaire, isolés.

Fig. ̈. Lescurs de l'oraire écartés les uns des antres à l'aide d'aiguilles pour montrer to rachis.

Fig. 6. Eufs pris datus la partie inférieure de lovaire.

Fig. 9-10. Prenier développement de l'embryon dans l'eui.

Fig. 11. Corpuseules séminan pris dans la vésicule séminale du mâle.

ligg. 12. Les mêtmes apries l'action de l'acide acétipne.

Fig. 13-14. Corpuscules sinninaux pris daus les organes gúnérateurs de la femelle.

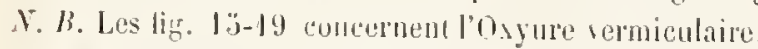

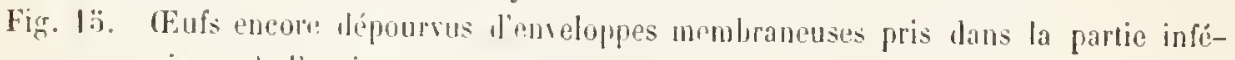
rieure de l'usaire.

Fig. 16. Ekuf inùr us de profil.

Fig. 17. Euf contenam un embryou, un de profil.

Fig. 18. Evuf contenant un embryou, vu par la face ventrale.

Fig. 19. Einf dont le churion a étri brisé par une pression artificielle.

\section{PIAXCHE 1}

N. B. Les figures 1-10 concernent l'érolution des zoospermes de l'A scaris suilla.

Fiğ. 1-8. Evolution des corpuscules séminaux dans l'intćrieur des organes générateurs mảles. 
Hig. 9. Corpusenles séminaux d’un mâte non adulte.

Fig. 10. Curpuscules séminaux pris dans les organes générateurs des femelles.

Figr. 11. Corpuscules séminum pris ehez me Ascaris Mystur femolle.

V. B. Les ligures 12-19 ont rapport à l'évolution des corpusenles séminaux du strongylus auricularis.

Fig. 12-21. Evolution des corpuscules sémimun daus les organes màles.

Fig. 22-99. Evolulion des corpusenles séminau dans les organes généraleurs lemelles.

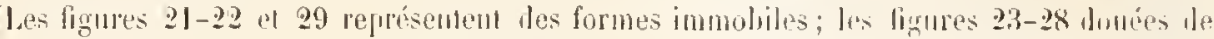
mouvements amolyens.

\section{PIANGIIE VI.}

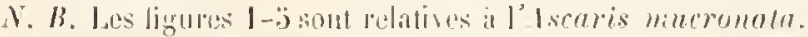

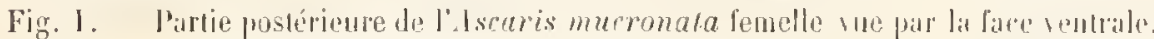

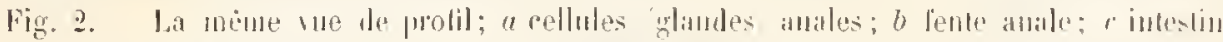
lapiseé par son épillélium; d boyaus celluleur; e, f cellules musendaires servant

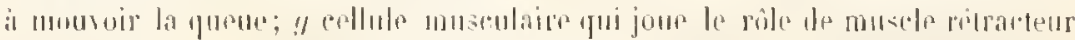
de l'illis.

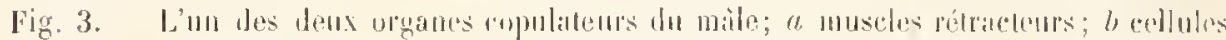
munies d'un nucléns a ; a spicule; $k$ rellules musculaires.

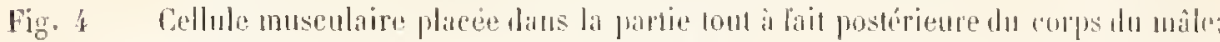

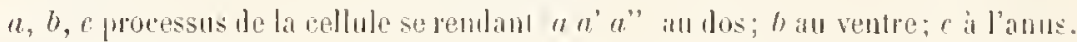

Fig. :j. Jartie inféripure du lube génératenr mâle; a paroi mune de son épithélium; b corpuscules séminaus.

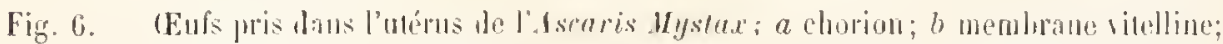
$c$ vitellus.

\section{PLANCIIE VII.}

N. B. Les digures 1-10 concenent l'1 searis commulata.

Fig. 1. Partio postúrienre du mile vie fill dessous; $a$ cellules (glamles) anales; $b$ anus; e spienles; d intestin; fartes membraneuses.

Fig. 2. Partie supérience du testicule.

fig. 3. Corpuscules sémintux pris dans le lesticule dans le canal déférent.

lig. 4. Les mines cellules dans l'acle de la prolifíration.

Fig. ̈̈. Corpuseules síminaur pris dans la vésienle séminale du mầle.

Fìg. 6. Corpuscules séminaus à mourements amobéens pris dans la poche séminale de la femelle.

Jig. 7. Blastogene.

Fig. 8. Fragumont de la partie suprérienre du vitellogene.

Fig. 9. Partic de la région infúrieure du vitellogine. 
Fig. 10. La mème, modifićo par l'action de l'eau salće.

X. B. Les ligures 11-1̈̈ cuncernent le Strongylus auricularis.

Fig. 11. Blaslugine.

Fìg. 12. Parlie de la régrion supéricure du vitellogène.

Fig. 13. Parlic de la région moyenue du vitellogène.

Fig. It. Partie de la régrion inférieure du vitellogiene.

Figr. lis. Parlic de l'oviducte rempli d'oufs.

\section{PIANCHE VIII.}

lig. 1. Chorion de l'auf de l'lledruris androphora, four montrer l'ouserture dont il ast muni à chaque poile.

Fig. 2-7. Stades divers de la segmentation des cutls d'lledruris androphora.

lig. 8. Euf d'lledruris androphora contenant un embryou.

Fig. 9-10. Eufs de l'Ascris spirotheca munis de leur chorion spiral.

Fig. 11. Blastogene d'une jeune Avearide trouvie dans l'intestiu du Triton teniatus.

Fig. 12. Muscles lungitudinaux des parois du corps de cette même Ascaride.

ling. 13. Huscles longitudinaux des parois du corps de l'.lscuris mucronata. 



\section{REM A R QUE.}

Dans un travail inséré dans le numéro doelobre 1858 de la Zeitschrift für wiss. Zoologie, M. Munk ${ }^{2}$ se plaint amèrement de ce que je ne faise aucune nention de ses recherehes dans la "Vorläufige Mlittheilung " yue jai publiée en 1857 daus ce même journal. Il lait observer que ses recherches ont été faites en même temps que les miennes, qu'elles ont été présentées an même concours. et que le rapport de la commission annonee expressément que ces deux travaux sont arrivés, sur la plupart des points, aux mêmes résnltats. l'espère tranquilliser la légitime, susceptibilité de II. Munk, en rappelant que ma "Vorthiufige Mittheilung" a été adressée en Ilai 1857 i la Zeilschrift für miss. Zooloyie, et que la commission n'ayant rapporté sur le travail de M. Mtrnk qu'au ınois d'Août de la même année, il m’était impossible de connaître alor's les conclusıons, ni l'existence de ce travail. te me plais toutefois i reconnaitre ici que les recherches de ll. Munk, faites à la même époque yue les miennes, ont conduit leur auteur à des résultats en très-grande partie semblables aux miens. Il est inutile de mappesantir ici sur les points de détail qui présentent quelque divergence, d'autant plus que M. Mtrnk, me blâmant d'avoir it peine abordé certains sujets, trouvera une réponse satisfaisante dans les pages qui précèdent. Il est clair que dans ma "Vorläufige Mlttheilung " je ne pouvais entrer dans une foule de détails qui trouvent lenr place dans le mémoire complet.

1 Leber Ei-und Samenbitdung und Befruelıtumg bei den Nemaloden, von Hermann Hunk. 


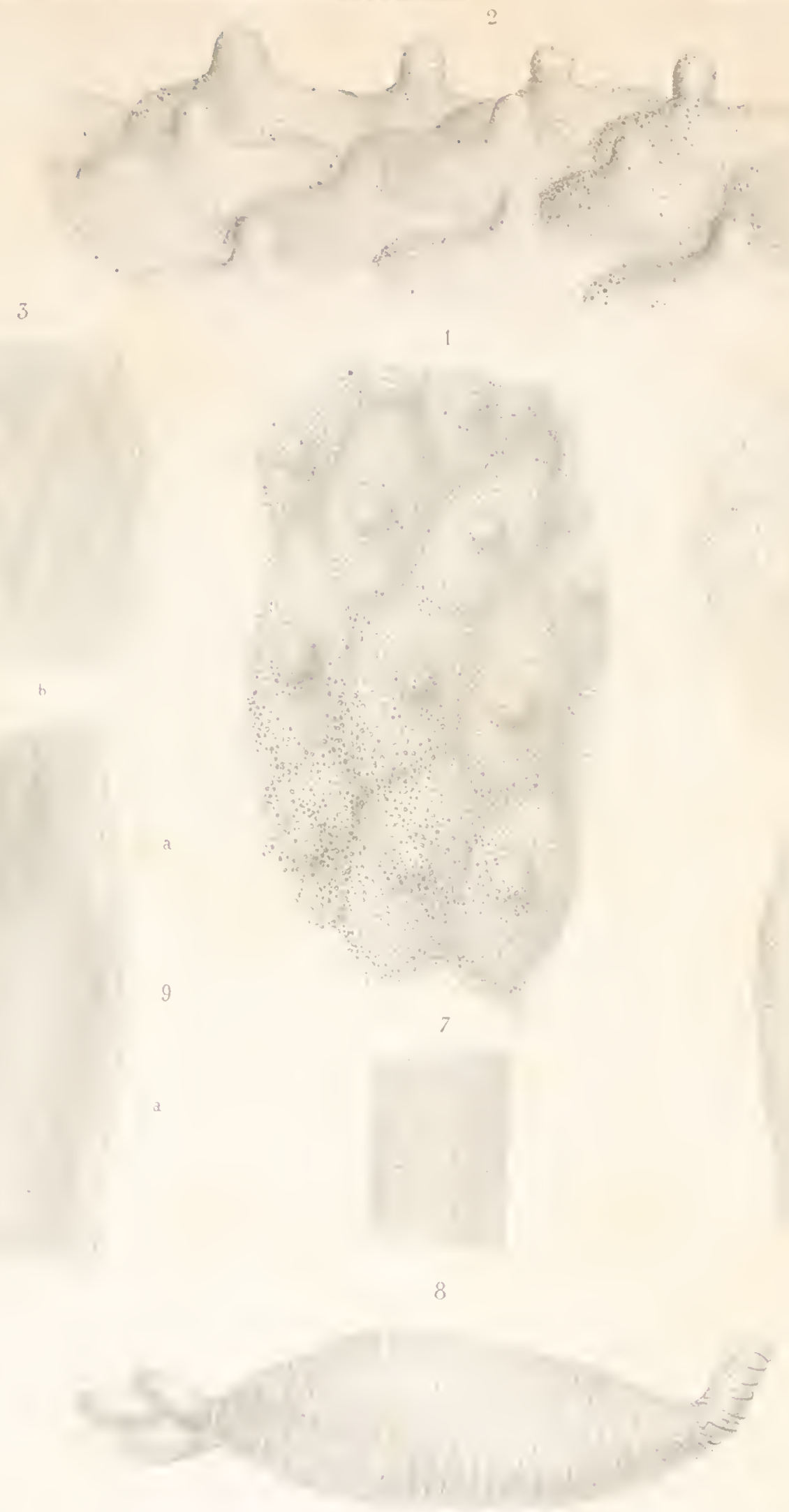



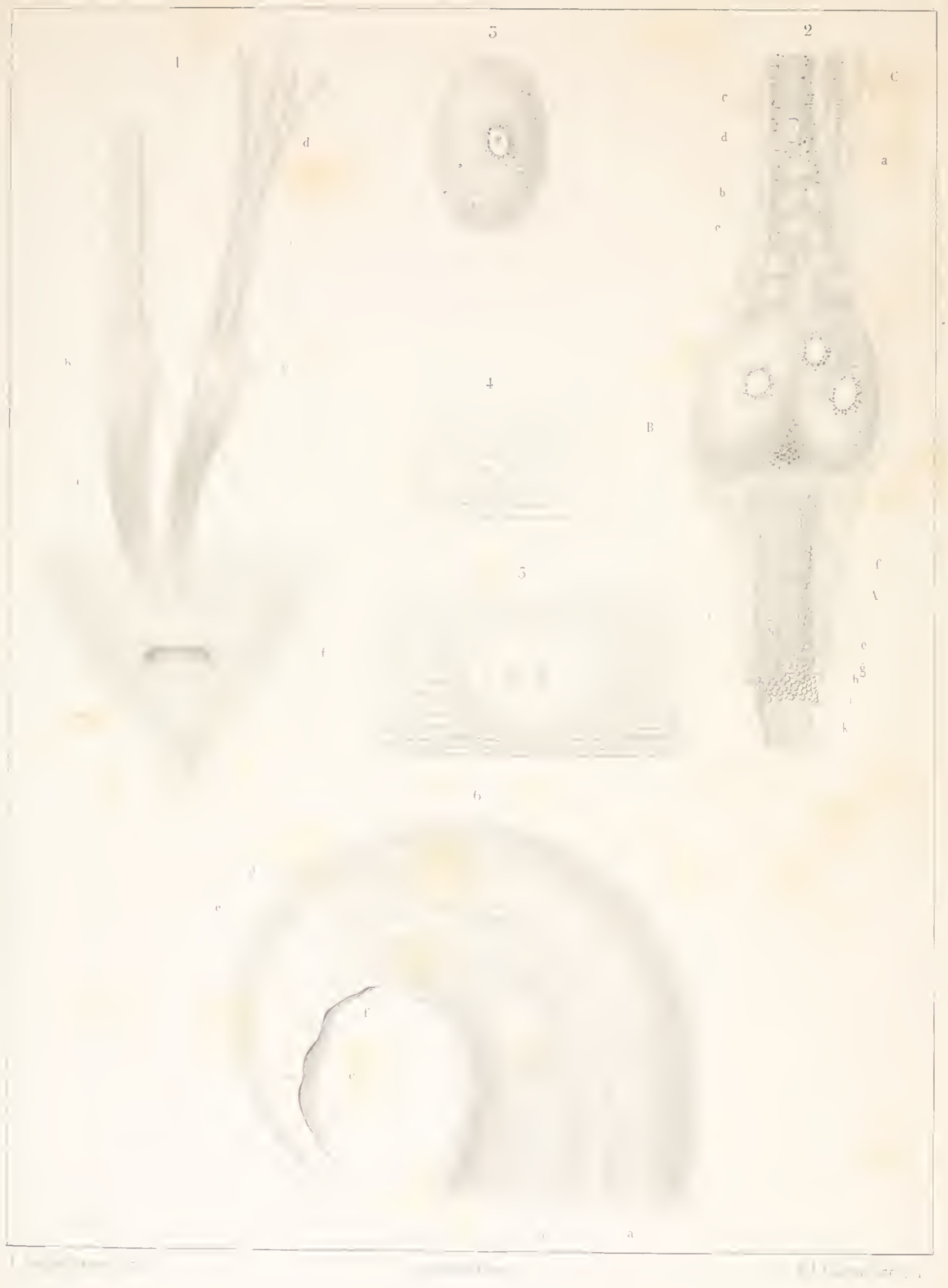





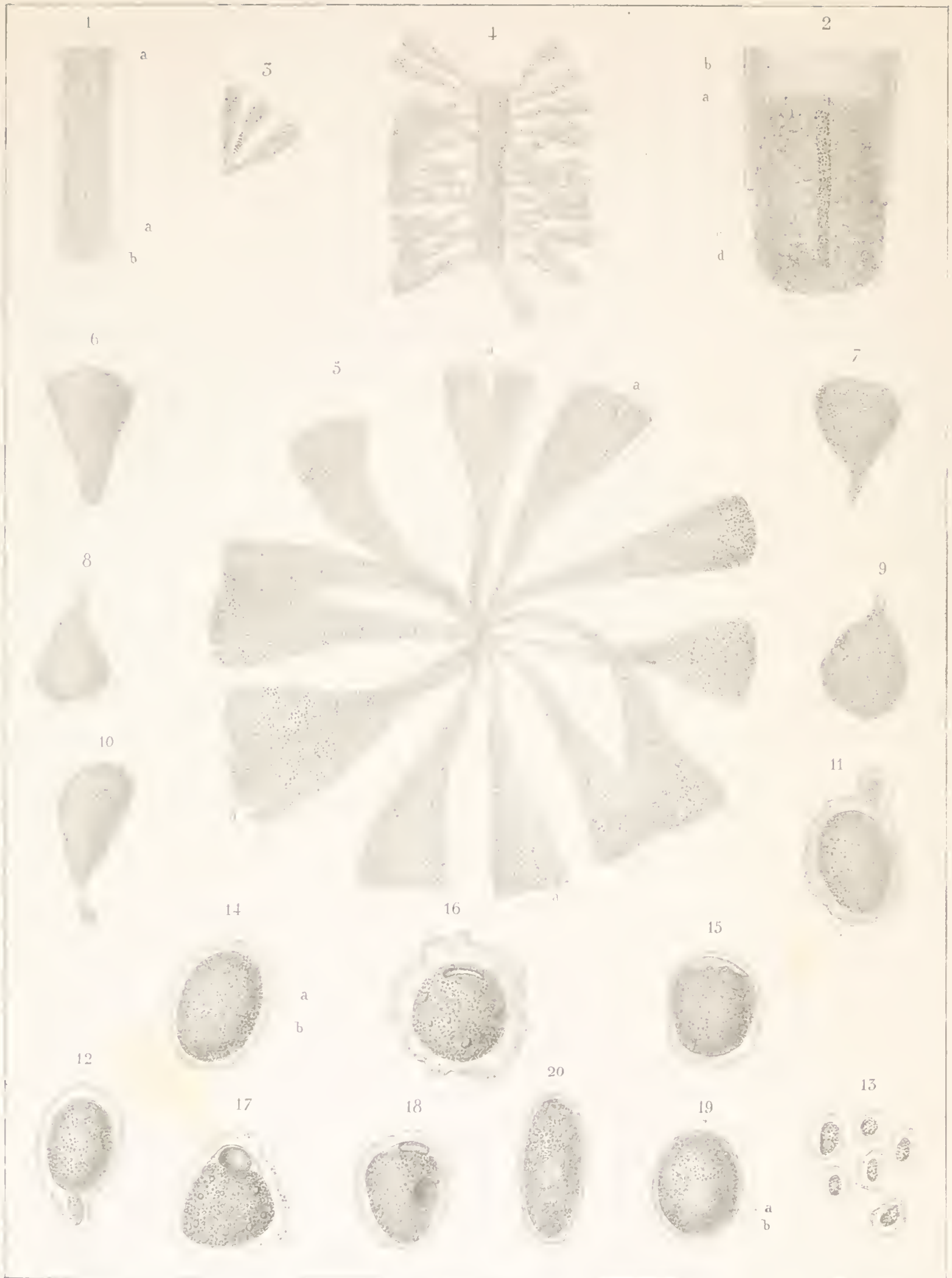

Ed Clapar.... do t 



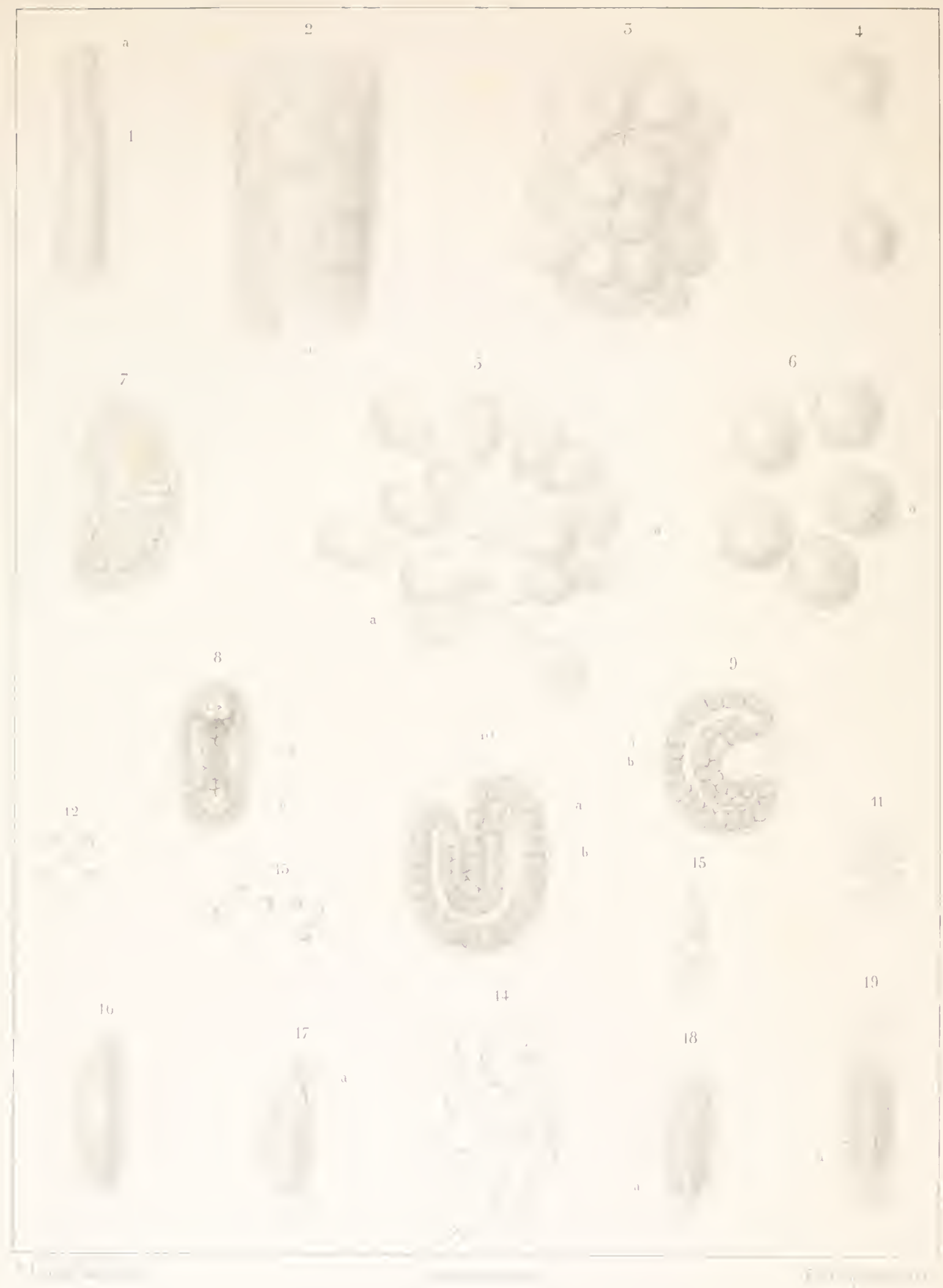






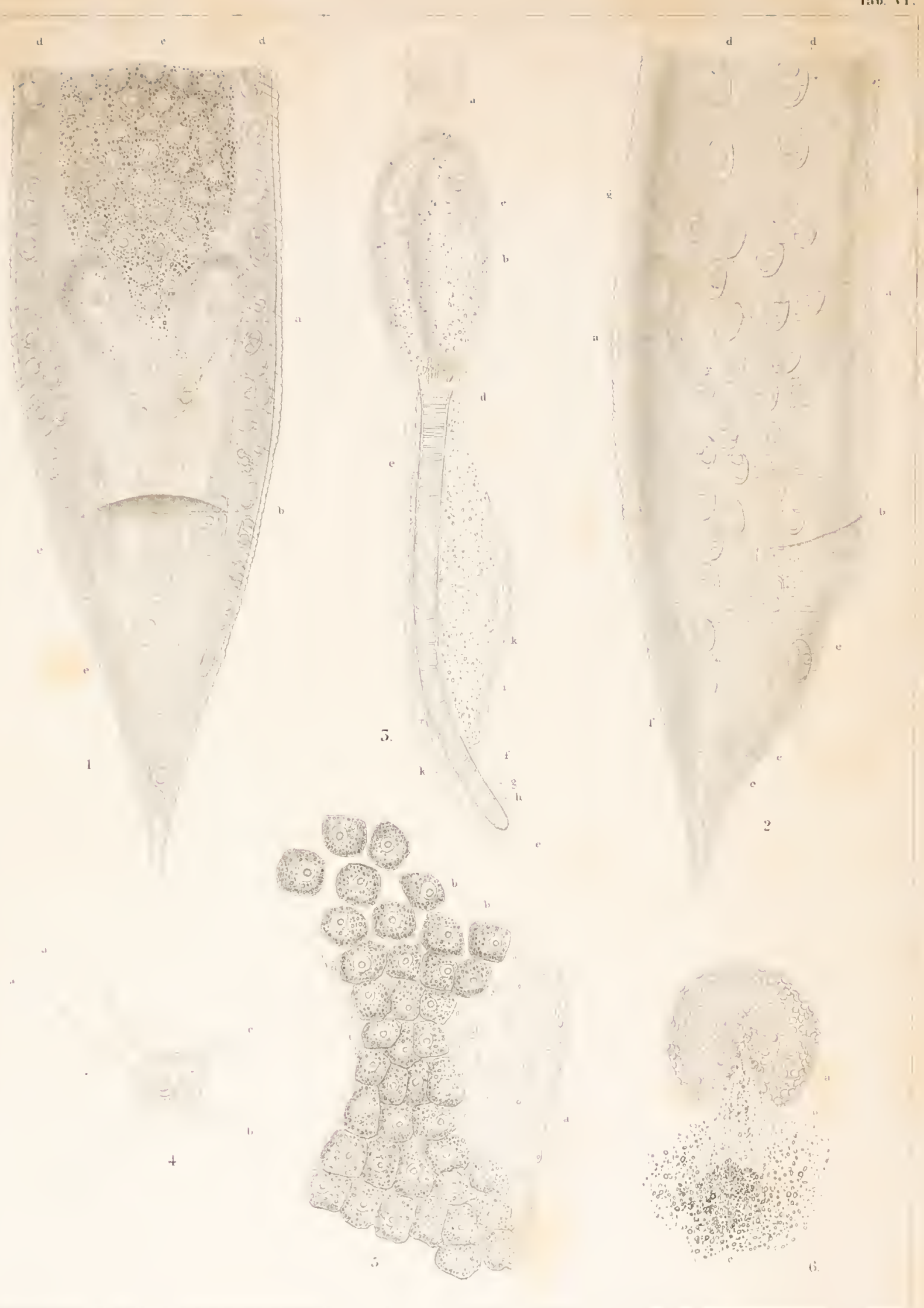


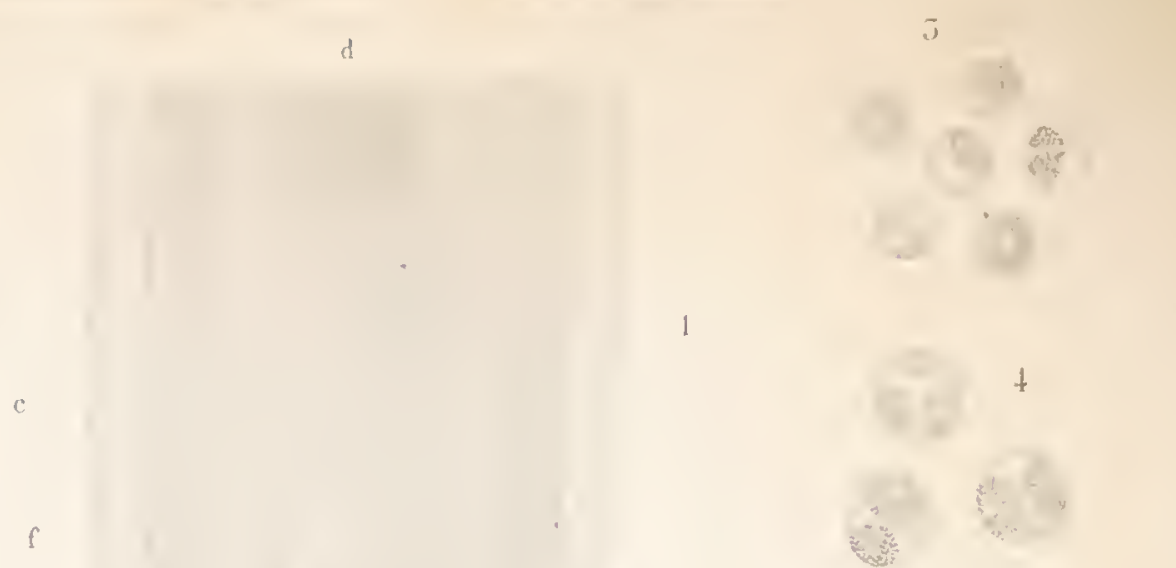

1
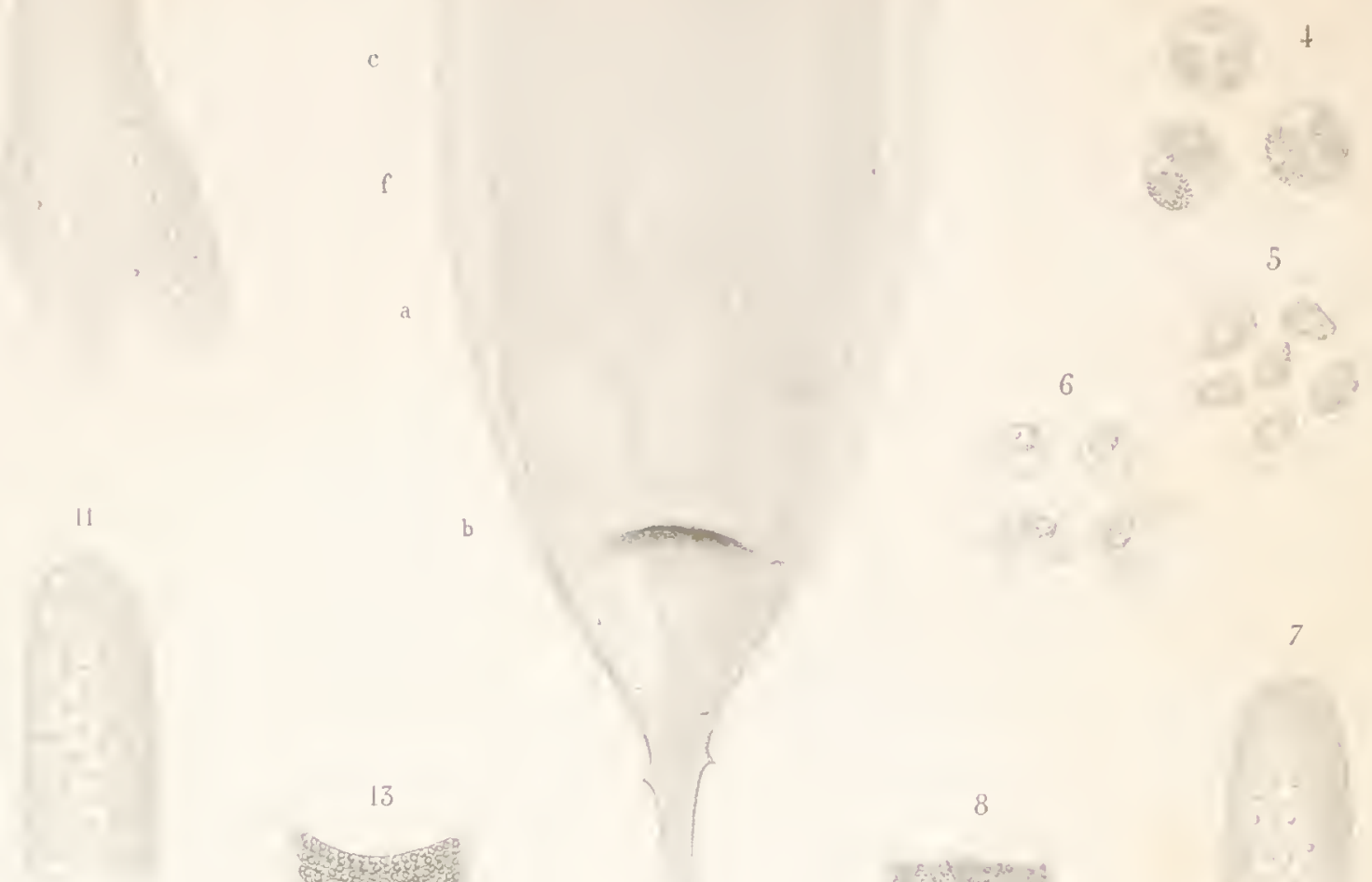

b
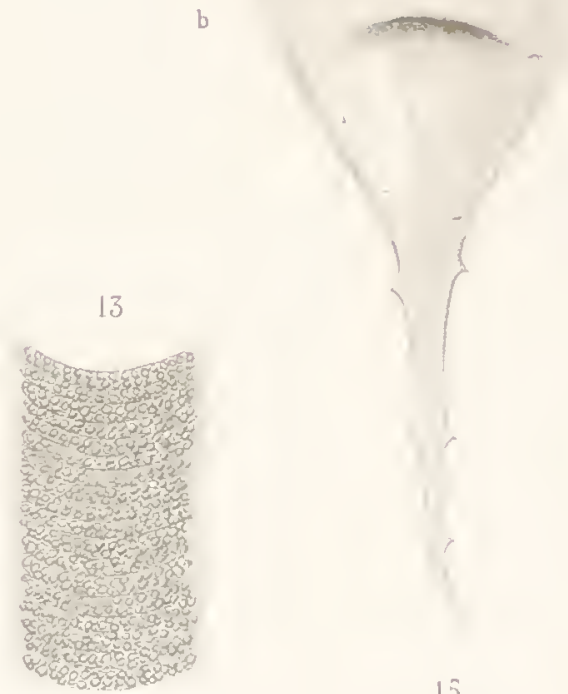

8

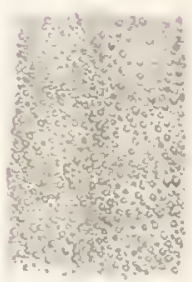

15
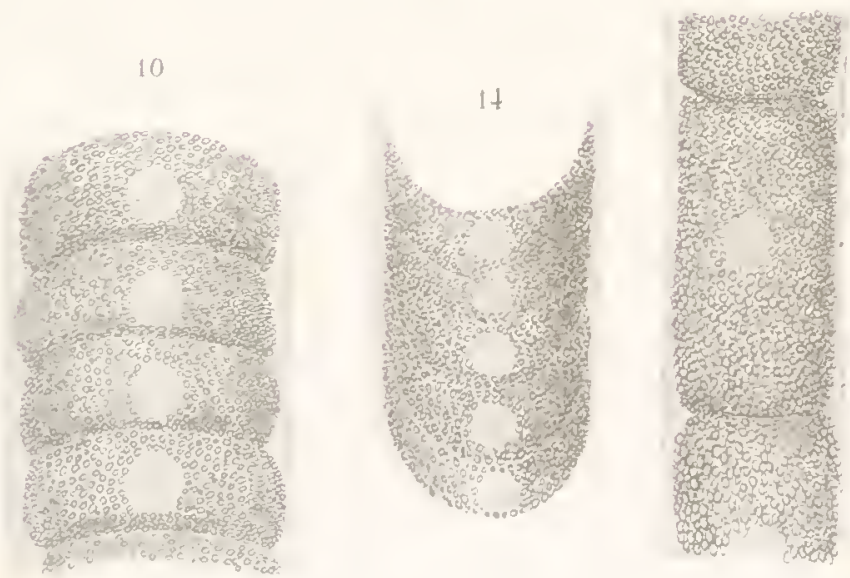

9

12
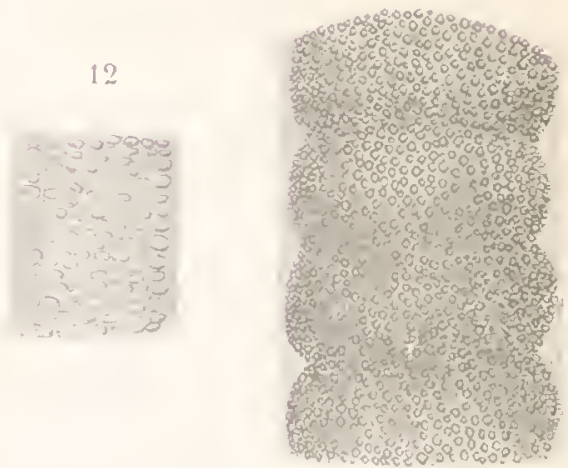
. 


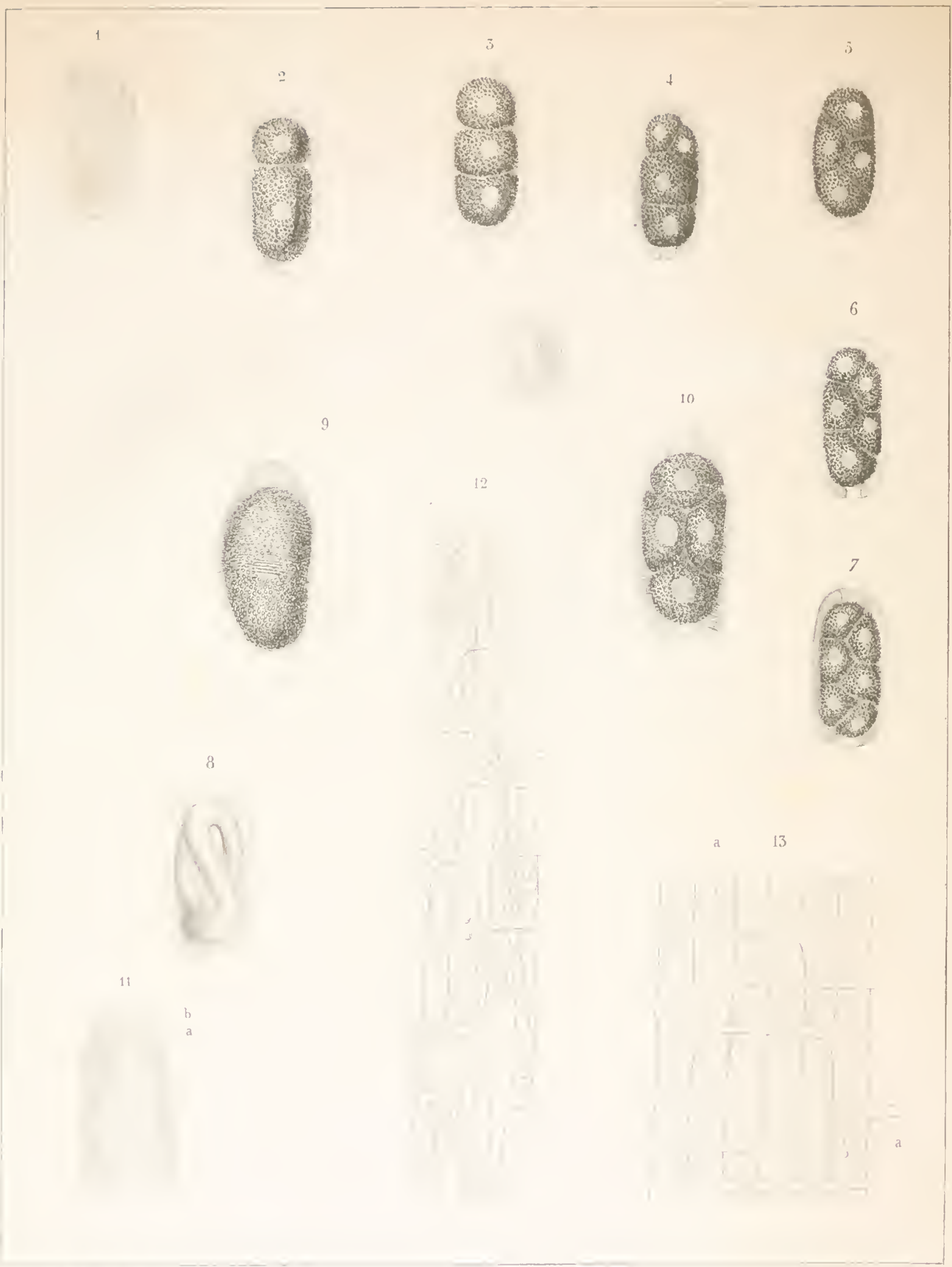


Printed in Sw.Lerland 


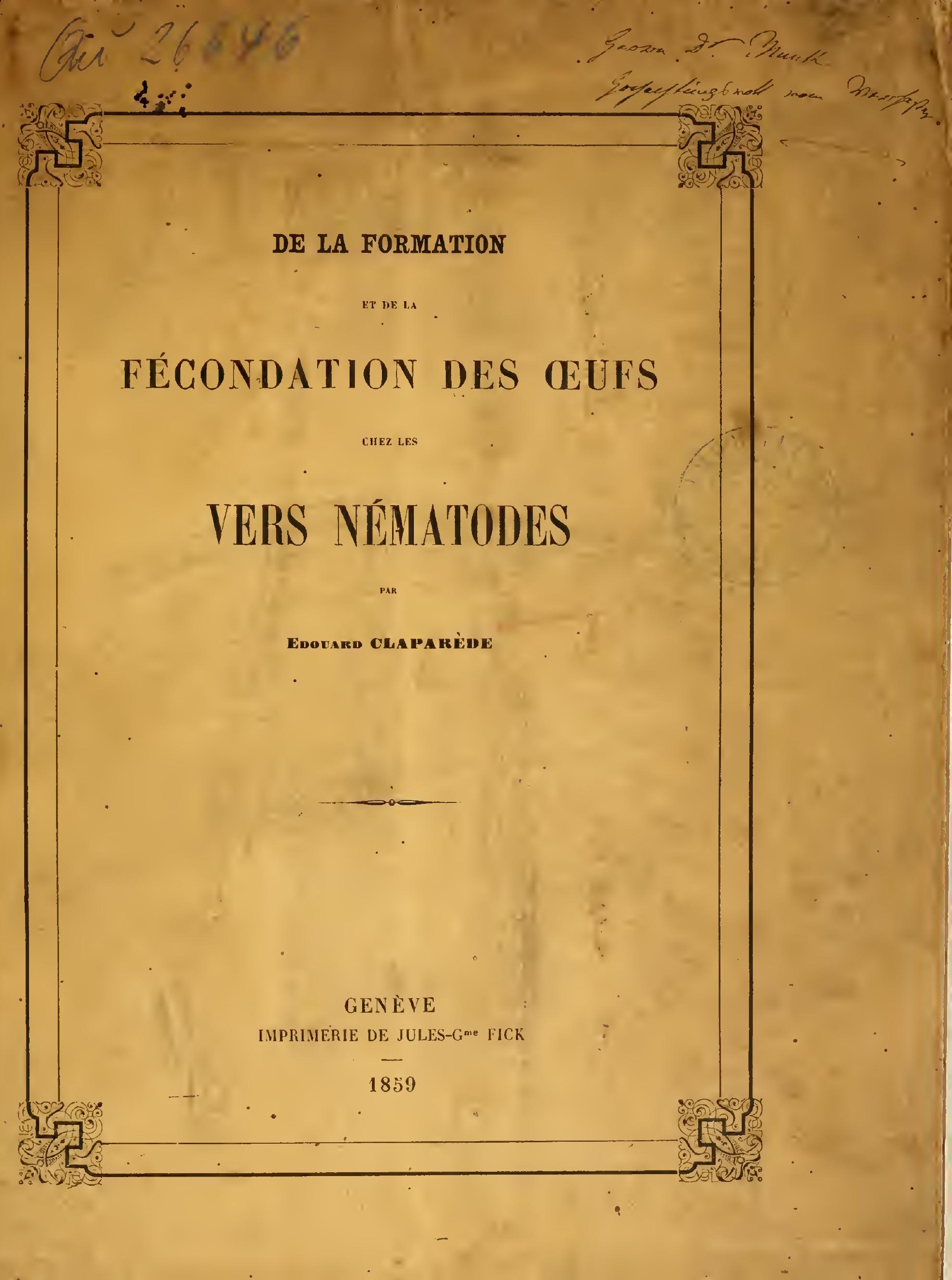






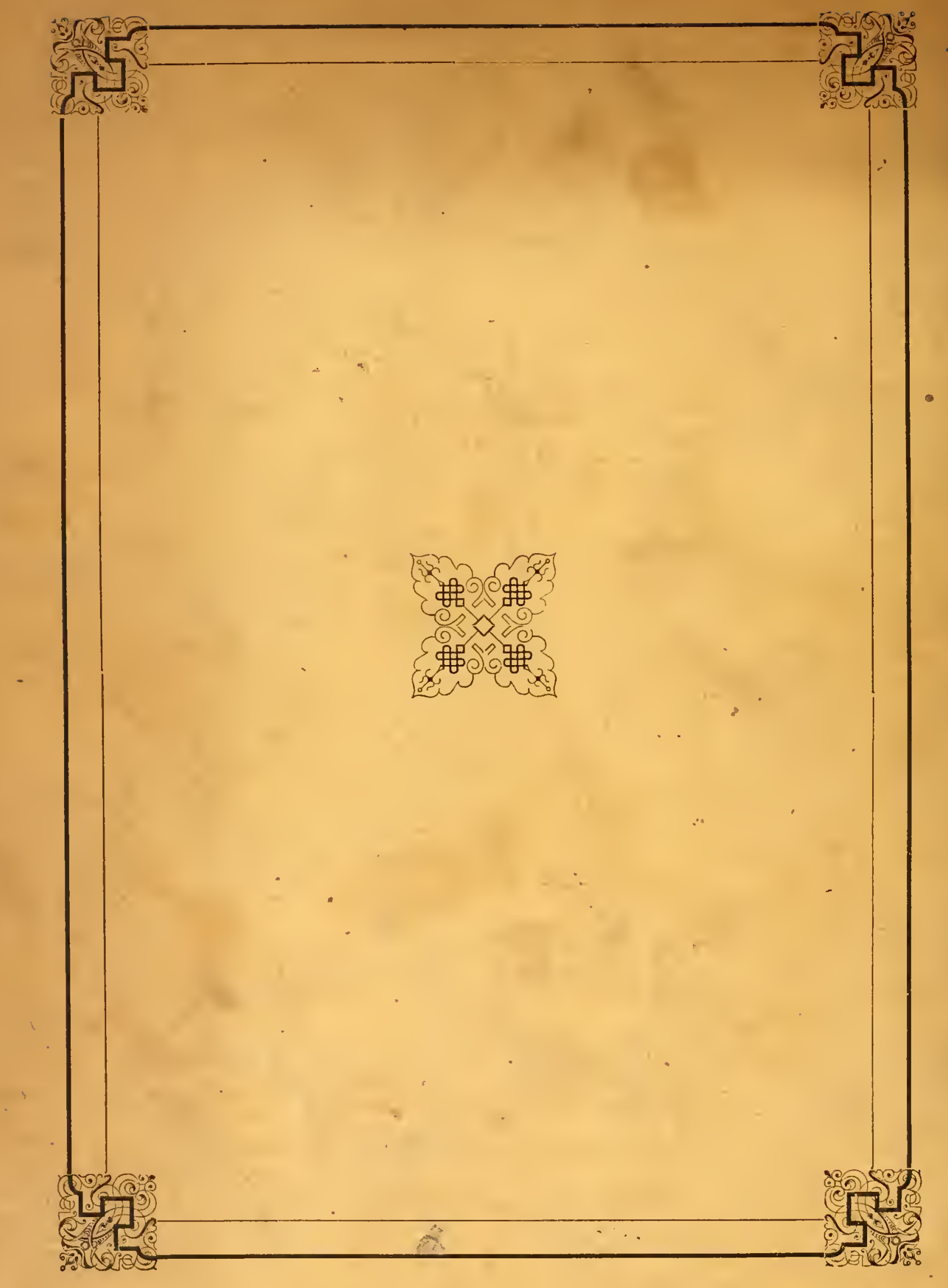






